\title{
Indian Pucciniales: taxonomic outline with important descriptive notes
}

\section{Gautam AK ${ }^{1}$, Avasthi $S^{2}$, Verma RK ${ }^{3}$, Devadatha $B^{4}$, Jayawardena RS ${ }^{5}$, Sushma ${ }^{6}$, Ranadive $\mathrm{KR}^{7}$, Kashyap $\mathrm{PL}^{8}$, Bhadauria $\mathbf{R}^{2}$, Prasher $\mathrm{IB}^{9}$, Sharma $\mathrm{VK}^{3}$, Niranjan $\mathbf{M}^{4,10}$, Jeewon $\mathbf{R}^{11}$}

\author{
${ }^{1}$ School of Agriculture, Abhilashi University, Mandi, Himachal Pradesh, 175028, India \\ ${ }^{2}$ School of Studies in Botany, Jiwaji University, Gwalior, Madhya Pradesh, 474011, India \\ ${ }^{3}$ Department of Plant Pathology, Punjab Agricultural University, Ludhiana, Punjab, 141004, India \\ ${ }^{4}$ Fungal Biotechnology Lab, Department of Biotechnology, School of Life Sciences, Pondicherry University, Kalapet, \\ Pondicherry, 605014, India \\ ${ }^{5}$ Center of Excellence in Fungal Research, Mae Fah Luang University, Chiang Rai, 57100, Thailand \\ ${ }^{6}$ Department of Botany, Dolphin PG College of Science and Agriculture Chunni Kalan, Fatehgarh Sahib, Punjab, India \\ ${ }^{7}$ Department of Botany, P.D.E.A. 's Annasaheb Magar Mahavidyalaya, Mahadevnagar, Hadapsar, Pune, Maharashtra, \\ India \\ ${ }^{8}$ ICAR-Indian Institute of Wheat and Barley Research (IIWBR), Karnal, Haryana, India \\ ${ }^{9}$ Department of Botany, Mycology and Plant Pathology Laboratory, Panjab University Chandigarh, 160014, India \\ ${ }^{10}$ Department of Botany, Rajiv Gandhi University, Rono Hills, Doimukh, Itanagar, Arunachal Pradesh, 791112, India \\ ${ }^{11}$ Department of Health Sciences, Faculty of Medicine and Health Sciences, University of Mauritius, Reduit, Mauritius
}

Gautam AK, Avasthi S, Verma RK, Devadatha B, Jayawardena RS, Sushma, Ranadive KR, Kashyap PL, Bhadauria R, Prasher IB, Sharma VK, Niranjan M, Jeewon R 2021 - Indian Pucciniales: taxonomic outline with important descriptive notes. Mycosphere 12(1), 89-162, Doi 10.5943/mycosphere/12/1/2

\begin{abstract}
Rusts constitute a major group of the Kingdom Fungi and they are distributed all over the world on a wide range of wild and cultivated plants. It is the largest natural group of plant pathogens including $95 \%$ of the subphylum Pucciniomycotina and about $8 \%$ of all described Fungi. This article provides an overview and outline of rust fungi of India with important descriptive notes. After compilation of available literature on Indian rust fungi from various sources, it was observed that these fungi are distributed in 16 families, 69 genera and 640 species. They belong to Coleosporiaceae, Crossopsoraceae, Gymnosporangiaceae, Melampsoraceae, Milesinaceae, Ochropsoraceae, Phakopsoraceae, Phragmidiaceae, Pileolariaceae, Pucciniaceae, Pucciniastraceae, Raveneliaceae, Skierkaceae, Sphaerophragmiaceae, Tranzscheliaceae and Zaghouaniaceae. There are still many rust fungi with uncertain taxonomic position, and they have been referred to incertae sedis. The placement of all fungal genera is provided at the class, order and family-level along with number of species in a genus. Notes for each rust family along with total Indian records and other taxonomic information on transferred genera and species are also presented. A phylogenetic analysis from a combined LSU and ITS dataset for 25 rust genera is presented to provide a better understanding of their phylogeny and evolution.
\end{abstract}

Key words - India - Phylogeny - Pucciniomycotina - rust fungi - Systematics

\section{Introduction}

Rust fungi (Basidiomycota, Pucciniales) are a highly diverse group of obligate biotrophic parasites, distributed in all geographical areas on a wide range of wild and cultivated plants ranging 
from ferns, Gymnosperms and Angiosperms (Duplessis et al. 2011). They are called rusts as one of their spore types i.e. the urediniospores and (uredinia) on host surface are often rust coloured. These fungi are unique and fascinating group of organisms possessing diverse structures (spermogonia, two anamorphs, teleomorphs and basidiospores) in their life cycle. Besides having up to five or six morphologically and functionally distinct spore types, many rust fungi exhibit complicated life cycles with variable host plant specificity. Because of their obligate parasitic nature, actively growing rusts survive only on living hosts and produce teliospores towards the end of the growing season. Species of Pucciniomycotina show simple life cycle (simple teliosporic yeasts) to the complex elaborate fivestage life cycles of the biotrophic rust fungi, the latter regarded as the most complex organisms in Kingdom Fungi (Lutz et al. 2004). Along with various spore types, some rust fungi require alternation between two exclusive and unrelated host plant taxa to complete their life cycle (heteroecious rust), although others can complete their life cycle on a single host plant (autoecious rust) (Kolmer et al. 2009). Parasitism and host specialization is highly developed in rust fungi (Savile et al. 1971, Duplessis et al. 2011). They constitute one of the major groups of plant pathogenic fungi. Puccininales is the most speciose order in Pucciniomycotina and include $95 \%$ of the subphylum and ca. $8 \%$ of all described Fungi (Kirk et al. 2008).

The rust fungi have a long research history, mostly due to their economic importance in agriculture and forestry, and easily noticeable symptoms (Cummins \& Hiratsuka 2003). Taxonomically the rust fungi belong to Pucciniomycotina, one of the three subphyla of Basidiomycota. Further, these fungi are placed in class Pucciniomycetes and order Pucciniales. In some systems of classifications, the rust fungi are placed in the subclass Heterobasidiomycetes based on basidial morphology while in other systems, they are with smut fungi in Teliomycetes based on their similar basidial characteristics (Talbot 1971, Webster 1980). Different morphological characters have been emphasized in earlier system of rust taxonomy and classifications (Ono \& Hennen 1983). Initially, rust fungi were classified into three (or four) families, Melampsoraceae, (Coleosporiaceae), Pucciniaceae and Zaghouaniaceae based on the characteristics of basidia and teliospores (Sydow \& Sydow 1915, Cunningham 1931). Classification of rust fungi in subfamilies or tribes and morphology of telia in taxonomy was also considered (Sydow \& Sydow 1915, Dietel 1928). Use of morphology of spermogonial (0) and aecial (I) stages was emphasized by Hiratsuka \& Cummins (1963) and Hiratsuka (1983) in the classification of rust fungi. The morphological characters of telium (Thirumalachar \& Cummins 1949) and teliospores (Dietel 1928, Thirumalachar \& Cummins 1948, Thirumalachar \& Mundkur 1949) played an important role in the taxonomic placement of rust fungi (Alexopoulus 1962). However, with the combination of different character of uredinia, aecia, type of spermogonia and telia, there has been several conflicting taxonomic hypotheses with time and this led to the proposal of 13-family system of classification of rust fungi. Based on telial morphology, three families were accepted: Melampsoraceae (sessile teliospores formed in columns in the telium), Pucciniaceae (stalked teliospores, produced in a single layer in the telium) and Coleosporiaceae (do not form a promycelium but karyogamy and meiosis occur directly within the teliospores that become septate during the germination process). Cummins \& Hiratsuka (1983, 2003) proposed 13 families. This was one of the the most accepted systems of rust classification till the incorporation of molecular data in systematic studies.

With the use of modern techniques (molecular studies) along with conventional methods (morphological studies), new taxonomic suggestions have been proposed regarding interrelationships of different groups of fungi. However, the earlier systems of classifications of rust fungi were mainly based on shape, size and other morphological characters of different spores and spore producing structures. Based on recent molecular techniques (DNA sequence data from the large ribosomal subunit) and ultrastructural investigations, it was observed that rusts are distantly related to some of the smuts (Aime et al. 2006, Kijpornyongpan et al. 2018). Subsequently Swann \& Taylor (1995a, b) and Swann et al. (2001) proposed the separation of class Urediniomycetes (the rust fungi), including the rusts (Uredinales), from class Ustilaginomycetes (the smut fungi) and class Hymenomycetes mushrooms and shelf or bracket fungi) under division Basidiomycota. Within the class Urediniomycetes, true rusts (Uredinales) account for over $95 \%$ of the species and more than $75 \%$ of 
the genera. Several research series on fungal diversity published in recent years provided an updated information on addition, exclusion, correct taxonomic position and transferred taxa of fungi. Aime (2006) examined representative species from the 13 families proposed by Cummins \& Hiratsuka (2003) and based on sequence analyses of the $18 \mathrm{~S}$ and $28 \mathrm{~S}$ nuclear rDNA regions, three major suborders were proposed Uredinineae, Melampsorineae and Mikronegeriineae. The Uredinineae includes species which produce the aecial stage on angiospermous host with pedicellate teliospores. Similarly, Melampsorineae comprises heteroecious, mostly macrocyclic rust species producing aecial stage on gymnosperms and producing sessile teliospores. Mikronegeriineae accomodates species with aecial stage usually on non-pine gymnosperms and in microcyclic types the teliospores functioned as urediniospores, with short pedicellate or sessile teliospores. He et al. (2019) presented notes, outline and divergence times of Basidiomycota. They differentiated Urediniomycetes from Basidiomycetes, as the nuclear membrane partially degrades during mitosis (semiopen pleuromitosis) in some Urediniomycetes while mitosis in basidiomycetes proceeds with preservation of the nuclear membrane (intranuclear pleuromitosis). They also differentiated rusts and smuts as non-basidiomata forming members of Basidiomycota, which comprises Pucciniomycotina and Ustilaginomycotina, respectively. They included eight families, Coleosporiaceae, Mikronegeriaceae, Phakopsoraceae, Phragmidiaceae, Pileolariaceae, Pucciniaceae, Raveneliaceae and Sphaerophragmiaceae in order Pucciniales, class Pucciniomycetes and subphylum Pucciniomycotina in their phylogenetic studies. However, they also presented the estimated number for taxa in Basidiomycota, of which, order Pucciniales possesses 15 families, 162 genera and 8105 species. Recently, Wijayawardene et al. (2020) outlined the kingdom Fungi up to genus level. They also presented 15 families (138 genera) under the order Pucciniales, class Pucciniomycetes and phylum Basidiomycota. It is interesting to mention here that the majority of Pucciniomycotina species (ca. 7500 of nearly 8500) belong to a single order Pucciniales that cause rust diseases in numerous plants (He et al. 2019, Wijayawardene et al. 2020, Aime \& McTaggart 2020). The recent higher-rank classification for rust fungi is provided by Aime \& McTaggart (2020), wherein they proposed the addition of four new suborders and seven new families, with some amendments in existing families. The classification of Pucciniales now comprises seven suborders and 18 families.

To understand the status of research on rust fungi in India, we have started this part here with the beginning of Indian mycological research. Here foreign visiting scientists or emigrant experts in the $18^{\text {th }}$ and $19^{\text {th }}$ centuries carried out most of the research. This started with the mycological studies initiated by K.R. Kirtikar in late $19^{\text {th }}$ century who collected and identified numerous fungi. Although the special credit to initiate mycological research in India goes to A. Barclay and E.J. Butler because of their contribution to initiate and organize research on mycology and plant pathology in India. After the establishment of the Imperial Agricultural Research Institute at Pusa (Bihar) during the year 1905 with the generous grant of 30,000 pounds from an American philanthropist, Mr. Henry Phipps, mycological research in India gained momentum. With the earlier efforts of E.J. Butler, the first imperial mycologists to the then British Government of India, a firm foundation of mycology and plant pathology was laid in this country and he is aptly referred to as the Father of Indian Mycology (Subramanian 1986). This research continued with the passage of time and involvement of many more mycologists. With the advancement in mycological research, B.B. Mundkur and M.J. Thirumalachar laid down a pioneer contribution in the studies of rust and smut fungi as Ustilaginales of India (Mundkar \& Thirumalachar 1952). Foundation of the Indian Phytopathological Society was also laid down by B. B. Mundkur along with S.R. Bose, both sereved as its earliest presidents. K.C. Mehta (1940) studied the problem of the recurrence of the wheat rust in plains of India. They mainly focused on cereal rust of India. Balchandra Bhavanishankar Mundkur and Mandayam Jeersannidhi Thirumalachar in 1952 jointly published a consolidated list of Indian Ustilaginales. Similarly, Thirumalachar \& Mundkar (1950) published a very useful appendix of genera of rust fungi. Simultaneously, Hans Sydow, a German mycologist, son of Paul Sydow also contributed a lot in understanding the Himalayan mycoflora including rust fungi. Similarly, George Baker Cummins, A. Barclay and Joseph Charles Arthur investigated the rusts occurring near North Western Himalayas. D.P. Mishra along with other mycologists also investigated rust fungi of agricultural crops in India. 
Several other mycologists contributed significantly to the development of research on rust fungi in India on a regional basis. Pioneering work of $\mathrm{C}$. Mohanan cannot be neglected as modern mycologists. He worked on biodiversity of plant pathogenic fungi of the Western Ghats and published a book entitled "Rust Fungi of Kerala, India". Some names worth mentioning are Ramesh Chand Sharma, Sanjeev Sharma, R.K. Sharma, S.N. Sachan and Ajay Kumar Gautam, who actively worked in the past and are still engaged in studies of various aspects of rust fungi of Himachal Pradesh. Dr. E.J. Butler \& G.R. Bisby compiled a monograph "The Fungi of India", in 1931. The fungi of India series have been revised from time to time and updated by several workers. However, the contribution of great Indian mycologists K.D. Bagchee, T.S. Ramakrishnan, J.H. Mitter, K.J. Narsimhan, S.N. Das Gupta, R.N. Tandon, R. Prasad, T.S. Sadasivan, C.V. Subramaniam and many more cannot be ignored. The taxonomic research on rust fungi in India has been based primarily on morphology of certain spore stages. Only a few studies published recently have employed modern tools and techniques for identification of rust fungi. Several institutes like Indian Type Culture Collection (ITCC) New Delhi; National Fungal Culture Collection of India (NFCCI) Pune, Maharashtra; CSIR-IMTECH Chandigarh, NBAIM Mau and many more are actively carrying research on fungal taxonomy and other related aspects and providing facilities for molecular characterisation of fungi including rust fungi in India. A major scientific breakthrough of the Indian Council of Agricultural Research (ICAR) scientists lead to the decoding of genomes of 15 strains of wheat rust fungus Puccinia triticina. Herbarium Cryptogamae Indiae Orientalis (HCIO) has a rich collection of rusts, smuts, powdery mildews and meliolales fungi and has more than 3500 type specimens (Maheswari et al. 2012). HCIO documented a comprehensive checklist of Puccinia species of India along with herbarium photographs and brief description (Kamil et al. 2013). Similarly, "Rust fungi of Kerala, India" (Mohanan 2010); First checklist of rust fungi in the genus Puccinia from Himachal Pradesh, India" (Gautam \& Avasthi 2016a); and "A checklist of rust fungi from Himachal Pradesh, India" (Gautam \& Avasthi 2019) are the recent compilations of rust fungi from India. In subsequent years, a plethora of articles on Pucciniales (Uredinales) has been published, which has added a lot to understanding this group of fungi, thus it is essential and pragmmatic to compile it into a single document. Therefore, we are starting a series of publications on rust fungal diversity from India, and this first paper provides basic information and the latest trends related to taxonomic outline of rust fungi of India with important descriptive notes.

\section{Materials \& Methods}

\section{Layout of the paper}

A brief description of each genus up to its higher taxonomic rank related with rust fungi of India is provided. During the listing of genera, their species, and other higher taxonomic ranks into a single outline, all generic names are listed from previously published literature pertaining to rust fungi of India. The detailed literature in reference to Indian rust fungi is summarised in the present study under the heading "literature used during the study of rust fungi". The names of some species have been replaced by currently accepted names after consultation of MycoBank (www.mycobank.org)/ and Species Fungorum (www.speciesfungorum.org) websites and this has been indicated in such cases. For general outline of Indian rust fungi, we adopted He et al. (2019) and Wijayawardene et al. (2020). Aime \& McTaggart (2020) was followed to provide a higher-rank classification of rust fungi. To confirm their scientific entity where some generic/species names have been updated with currently accepted name, more literature on rust fungi was consulted (Cummins \& Hiratsuka 2003, Aime 2006, Aime et al. 2018, Aime \& McTaggart 2020. After complete verification, accepted taxa of rust fungi of Basidiomycota up to species level are presented in detailed. The existing and currently accepted names of various genera and species of rust fungi are provided as a separate section in this manuscript.

\section{Phylogenetic analyses}

Most of the rust fungi reported from India were characterized mainly based on the 
morphological characters of uredia and telia or other successive stages observed on collected samples. However, rust fungi reported from India lack molecular studies.

Based on earlier studies on Indian rust fungi, a checklist was prepared and the DNA sequence data from the LSU and ITS rDNA regions available for same rust fungi reported from other countries were downloaded from GenBank and through published literature (Maier et al. 2003, Aime 2006, Aime et al. 2006, Aime \& McTaggart 2020). Individual nucleotide sequences of LSU and ITS were aligned distinctly using MAFFT 7 (http://mafft. cbrc.jp/alignment/server/) (Katoh \& Standley 2013) and then manual checking and editing where necessary in BioEdit v.7.0.9 (Hall 1999). The ITS sequences of taxa containing weak aligned portions, incomplete data, missing sequence data and gaps were removed. The separate aligned gene regions of LSU and ITS were combined in BioEdit. The combined multigene sequence alignment was converted to PHYLIP format (.phy) using ALTER (alignment trans-formation environment: (http://sing.eiuvigo.es/ALTER/; 2021) for randomized accelerated maximum likelihood (RAxML) analysis. The aligned LSU and ITS single gene datasets and a concatenated dataset of LSU and ITS genes were analyzed with maximum likelihood using the RAxML-HPC2 on XSEDE (8.2.8) (Stamatakis et al. 2008, Stamatakis 2014) in the CIPRES Science Gateway platform (Miller et al. 2010) using GTR+I+G model of evolution. Maximum Likelihood bootstrap values greater than $70 \%$ were given above each node. Phylogenetic trees were visualized with FigTree v1.4.0 program (Rambaut 2012) and reorganized in Microsoft power point (2016).

Table 1 GenBank and voucher/culture collection accession numbers of species included in the phylogenetic study

\begin{tabular}{|c|c|c|c|c|}
\hline \multirow[t]{2}{*}{ Taxon } & \multirow[t]{2}{*}{ Voucher/culture } & \multicolumn{2}{|c|}{\begin{tabular}{|l|} 
GenBank accession \\
Numbers
\end{tabular}} & \multirow[t]{2}{*}{ References } \\
\hline & & ITS & LSU & \\
\hline Cerotelium fici & LAH20019AM & -- & MK135779 & Ishaq et al. (unpublished) \\
\hline Chrysomyxa pirolae & 190CHP_PCG_DU2 & & GU049555 & Feau et al. (2011) \\
\hline Coleosporium asterum & MCA3077 & -- & MG907226 & Aime et al. (2018) \\
\hline Coleosporium bletiae & BSC1 & -- & MN108162 & Zhou (unpublished) \\
\hline Coleosporium campanulae & -- & -- & KP017565 & Tian et al. (unpublished) \\
\hline Coleosporium campanulae & LB09265/ZT_Myc_58002 & KY810467 & -- & Beenken et al. (2017) \\
\hline Coleosporium inulae & U717 & -- & MG907223 & Aime et al. (2018) \\
\hline Coleosporium inulae & LB09168/ZT_Myc_57996 & KY810470 & -- & Beenken et al. (2017) \\
\hline Coleosporium ipomoeae & JRH 485 & MF769644 & -- & $\begin{array}{l}\text { McTaggart \& Aime } \\
(2018)\end{array}$ \\
\hline Coleosporium ipomoeae & R232 & -- & EU851160 & Zuluaga et al. (2011) \\
\hline Coleosporium senecionis & LB08877/ZT_Myc_57995 & KY810472 & -- & \\
\hline Coleosporium senecionis & PDD 98309 & -- & KJ716348 & Beenken et al. (2017) \\
\hline Coleosporium xanthoxyli & KUS-F30013 & -- & MK530184 & Shin et al. (2019) \\
\hline Cronartium quercuum & CqvGa-1 & L76495 & -- & Vogler \& Bruns (1998) \\
\hline Cronartium ribicola & -- & KX963430 & -- & Kaitera et al. (2017) \\
\hline & & & AF426240 & Maier et al. (2003) \\
\hline Crossopsora ziziphi & BPI 877877 & -- & MG744558 & Souza et al. (2018) \\
\hline $\begin{array}{l}\text { Gymnosporangium } \\
\text { clavariiforme }\end{array}$ & LD 1019 & HM114220 & -- & Dervis et al. (2011) \\
\hline $\begin{array}{l}\text { Gymnosporangium } \\
\text { clavariiforme }\end{array}$ & HMAS:24626 & -- & KU342766 & Zhao et al. (2016) \\
\hline $\begin{array}{l}\text { Gymnosporangium } \\
\text { confusum }\end{array}$ & LD 1021 & HM114219 & -- & Dervis et al. (2011) \\
\hline $\begin{array}{l}\text { Gymnosporangium } \\
\text { confusum }\end{array}$ & 20140808B H22 & -- & KP261043 & $\begin{array}{l}\text { Fernandez \& Alvarado } \\
(2016)\end{array}$ \\
\hline Hyalopsora polypodii & BPI 893256 & KY798367 & -- & $\begin{array}{l}\text { Demers \& Castlebury } \\
\text { (unpublished) }\end{array}$ \\
\hline Hyalopsora polypodii & DB 1681 & -- & AY512852 & $\begin{array}{l}\text { Begerow et al. } \\
\text { (unpublished) }\end{array}$ \\
\hline
\end{tabular}


Table 1 Continued.

\begin{tabular}{|c|c|c|c|c|}
\hline \multirow[t]{2}{*}{ Taxon } & \multirow{2}{*}{ Voucher/culture } & \multicolumn{2}{|c|}{$\begin{array}{l}\text { GenBank accession } \\
\text { Numbers }\end{array}$} & \multirow[t]{2}{*}{ References } \\
\hline & & ITS & LSU & \\
\hline Kernkampella breyniae & BRIP:56909 & -- & KJ862346 & McTaggart et al. (2015) \\
\hline Kweilingia divina & MCA3493 & -- & MG907215 & Aime et al. (2018) \\
\hline $\begin{array}{l}\text { Leucotelium pruni- } \\
\text { persicae }\end{array}$ & -- & AB097450 & -- & Osaki (unpublished) \\
\hline Maravalia pterocarpi & HNCM1 & KU301795 & -- & Wang et al. (2016) \\
\hline Melampsora caprearum & GE14_3_2 & KY649193 & -- & Piskur (unpublished) \\
\hline Melampsora caprearum & NYS-F-003819 & -- & KU550033 & Zhao (unpublished) \\
\hline Melampsora epitea & -- & -- & DQ354564 & Aime (2006) \\
\hline Melampsora epitea & 1046MEE-SA-QC.1 & GQ479218 & -- & Vialle (unpublished) \\
\hline Melampsora euphorbiae & -- & EF192199 & -- & Aime (unpublished) \\
\hline Melampsora euphorbiae & BPI 863501 & -- & DQ437504 & Aime et al. (2006) \\
\hline $\begin{array}{l}\text { Melampsora } \\
\text { hypericorum }\end{array}$ & BPI 893298 & -- & KY798351 & $\begin{array}{l}\text { Demers \& Castlebury } \\
\text { (unpublished) }\end{array}$ \\
\hline $\begin{array}{l}\text { Melampsora } \\
\text { hypericorum }\end{array}$ & PDD 97325 & KJ716353 & -- & $\begin{array}{l}\text { Padamsee \& McKenzie } \\
(2014)\end{array}$ \\
\hline Melampsora populnea & 892MPO-PTA-FR3.1 & EU808037 & -- & Feau et al. (2009) \\
\hline Melampsora populnea & -- & -- & AY444786 & Pei et al. (2005) \\
\hline $\begin{array}{l}\text { Melampsora salicis- } \\
\text { albae }\end{array}$ & HMAAC4068 HMAAC4068 & -- & MK372199 & Wang (unpublished) \\
\hline $\begin{array}{l}\text { Melampsora salicis- } \\
\text { albae }\end{array}$ & $13125 \mathrm{~F}$ & FJ455128 & -- & $\begin{array}{l}\text { Eslami et al. } \\
\text { (unpublished) }\end{array}$ \\
\hline $\begin{array}{l}\text { Melampsoridium } \\
\text { betulinum }\end{array}$ & KR-M-0048135 & -- & MK302187 & Bubner et al. (2019) \\
\hline $\begin{array}{l}\text { Melampsoridium } \\
\text { betulinum }\end{array}$ & H 6034375 & KF031552 & -- & McKenzie et al. (2013) \\
\hline $\begin{array}{l}\text { Melampsoridium } \\
\text { hiratsukanum }\end{array}$ & 421 & KC313888 & -- & Blomquist et al. (2014) \\
\hline $\begin{array}{l}\text { Melampsoridium } \\
\text { hiratsukanum }\end{array}$ & KR-M-0048149 & -- & MK302188 & Bubner et al. (2019) \\
\hline Milesina exigua & KR-M-0050247 & MH908478 & MK302211 & Bubner et al. (2019) \\
\hline Milesina polypodii & KR-M-0043190 & -- & MK302190 & Bubner et al. (2019) \\
\hline Nyssopsora thwaitesii & AMH:9528 & KF550283 & -- & Baiswar et al. (2014) \\
\hline Ochropsora ariae & KR-M-42604 & KX228773 & KX228778 & Scholler et al. (2019) \\
\hline Phakopsora apoda & PDD 72076 & MG461668 & MG461668 & $\begin{array}{l}\text { Demers \& Castlebury } \\
\text { (unpublished) }\end{array}$ \\
\hline Phakopsora cingens & BRIP:55628 & -- & KP729474 & Maier et al. (2016) \\
\hline Phakopsora meibomiae & Brazil 82-1 & AF333501 & -- & Frederick et al. (2002) \\
\hline Phakopsora meibomiae & R188 & -- & EU851164 & Zuluaga et al. (2011) \\
\hline Phakopsora phyllanthi & 83 & KF528025 & -- & Beenken (2014) \\
\hline Phakopsora phyllanthi & BPI 843632 & -- & KY764084 & $\begin{array}{l}\text { Demers et al. } \\
\text { (unpublished) }\end{array}$ \\
\hline $\begin{array}{l}\text { Phakopsora ziziphi- } \\
\text { vulgaris }\end{array}$ & HMJAU8595 & MK296536 & MK296506 & Ji (unpublished) \\
\hline Phragmidium barclayi & HMAS-67281 & -- & MG669117 & Liu et al. (2018) \\
\hline $\begin{array}{l}\text { Phragmidium } \\
\text { brevipedicellatum }\end{array}$ & HMUT100463 & -- & KU059170 & Xu et al. (unpublished) \\
\hline Phragmidium butleri & HMAS-67841 & -- & MG669118 & Liu et al. (2018) \\
\hline Phragmidium fragariae & -- & -- & AF426217 & Maier et al. (2003) \\
\hline $\begin{array}{l}\text { Phragmidium } \\
\text { mucronatum }\end{array}$ & TFS01 & -- & KJ867552 & El-Deeb (unpublished) \\
\hline $\begin{array}{l}\text { Phragmidium } \\
\text { potentillae }\end{array}$ & BRIP:60089 & -- & KT199403 & McTaggart et al. (2016b) \\
\hline
\end{tabular}


Table 1 Continued.

\begin{tabular}{|c|c|c|c|c|}
\hline \multirow[t]{2}{*}{ Taxon } & \multirow[t]{2}{*}{ Voucher/culture } & \multicolumn{2}{|c|}{$\begin{array}{l}\text { GenBank accession } \\
\text { Numbers }\end{array}$} & \multirow[t]{2}{*}{ References } \\
\hline & & ITS & LSU & \\
\hline $\begin{array}{l}\text { Phragmidium } \\
\text { potentillae }\end{array}$ & HMJAU8609 & MK296538 & -- & Ji (unpublished) \\
\hline $\begin{array}{l}\text { Phragmidium rosae- } \\
\text { moschatae }\end{array}$ & BPI 893257 & -- & KY798368 & $\begin{array}{l}\text { Demers \& Castlebury } \\
\text { (unpublished) }\end{array}$ \\
\hline Pileolaria pistaciae & -- & MG860928 & KY314266 & Ishaq et al. (2020) \\
\hline $\begin{array}{l}\text { Puccinia actaeae- } \\
\text { agropyri }\end{array}$ & TUB 14959 & -- & DQ917746 & Maier et al. (2007) \\
\hline Puccinia antirrhini & BPI 910208 & -- & KY764090 & $\begin{array}{l}\text { Demers et al. } \\
\text { (unpublished) }\end{array}$ \\
\hline Puccinia arenariae & BPI 893275 & & KY798385 & $\begin{array}{l}\text { Demers et al. } \\
\text { (unpublished) }\end{array}$ \\
\hline Puccinia argentata & IMI 502182 & -- & KC433402 & $\begin{array}{l}\text { Tanner et al. } \\
\text { (unpublished) }\end{array}$ \\
\hline Puccinia bistortae & TUB 14964 & -- & DQ917697 & Maier et al. (2007) \\
\hline Puccinia brachypodii & HSZ0975 & GQ457303 & -- & Jin et al. (2010) \\
\hline Puccinia brachypodii & BRIP 59466 & -- & KX999868 & Marin-Felix et al. (2017) \\
\hline Puccinia bupleuri & BPI 910217 & -- & KY764100 & $\begin{array}{l}\text { Demers et al. } \\
\text { (unpublished) }\end{array}$ \\
\hline Puccinia canaliculata & U-77 & HQ412647 & & Deadman et al. (2011) \\
\hline Puccinia carthami & SAF 1 & AF064822 & & $\begin{array}{l}\text { Berthier-Schaad } \\
\text { (unpublished) }\end{array}$ \\
\hline Puccinia carthami & -- & & AY787782 & Deadman et al. (2005) \\
\hline Puccinia cenchri & BPI055581 & -- & KY575080 & $\begin{array}{l}\text { Demers \& Castlebury } \\
\text { (unpublished) }\end{array}$ \\
\hline $\begin{array}{l}\text { Puccinia chloridis- } \\
\text { incompletae }\end{array}$ & HSZ1393 & KM096427 & KM096427 & $\begin{array}{l}\text { Mahadevakumar } \\
\text { (unpublished) }\end{array}$ \\
\hline Puccinia chrysanthemi & HSZ2154 & KX369039 & -- & $\begin{array}{l}\text { Szabo \& Nguyen } \\
\text { (unpublished) }\end{array}$ \\
\hline Puccinia chrysanthemi & $\mathrm{R} 79 \mathrm{~F}$ & -- & HQ201322 & Alaei (unpublished) \\
\hline Puccinia circaeae & TUB 14969 & -- & DQ917716 & Maier et al. (2007) \\
\hline Puccinia coronata & -- & -- & DQ354526 & Aime et al. (2006) \\
\hline $\begin{array}{l}\text { Puccinia crepidis- } \\
\text { japonicae }\end{array}$ & BPI 893282 & -- & KY798360 & $\begin{array}{l}\text { Demers \& Castlebury } \\
\text { (unpublished) }\end{array}$ \\
\hline Puccinia cyperi & BRIP 60997 & KU296885 & KU296885 & McTaggart et al. (2016a) \\
\hline Puccinia dactylidina & PUR F15426 & JX533546 & JX533546 & Liu et al. (2013) \\
\hline Puccinia dioicae & DAR 77052 & EF635897 & -- & Morin et al. (2009) \\
\hline Puccinia drabae & R209-16555 & EU014054 & EU014055 & Alaei et al. (2009) \\
\hline Puccinia ferruginosa & IBA7553 & -- & AB190901 & $\begin{array}{l}\text { Engkhaninun et al. } \\
\text { (unpublished) }\end{array}$ \\
\hline Puccinia ferruginosa & TSH-R6237 & AB188126 & -- & $\begin{array}{l}\text { Engkhaninun et al. } \\
(2005)\end{array}$ \\
\hline Puccinia graminis & $72 \_2$ & -- & JQ688991 & Berlin et al. (2012) \\
\hline Puccinia heterospora & BPI 893307 & KY798362 & -- & $\begin{array}{l}\text { Demers \& Castlebury } \\
\text { (unpublished) }\end{array}$ \\
\hline Puccinia heterospora & BRIP 60937 & -- & KU296886 & McTaggart et al. (2016a) \\
\hline Puccinia heucherae & RHS5296/05 & -- & DQ359702 & Henricot et al. (2007) \\
\hline Puccinia hieracii & DAOM 240969 & -- & HQ317515 & Liu et al. (2015) \\
\hline Puccinia hordei & PDD:101656 & -- & KX985762 & $\begin{array}{l}\text { Padamsee \& McKenzie } \\
\text { (2017) }\end{array}$ \\
\hline Puccinia hydrocotyles & -- & -- & GU936635 & Zuluaga et al. (2011) \\
\hline Puccinia iridis & KUS-F23394 & -- & MK446720 & Choi et al. (2019) \\
\hline Puccinia iridis & KUS-F30180 & MK446718 & & Choi et al. (2019) \\
\hline Puccinia kuehnii & BPI 881011 & -- & HQ666891 & Saumtally et al. (2011) \\
\hline
\end{tabular}


Table 1 Continued.

\begin{tabular}{|c|c|c|c|c|}
\hline \multirow[t]{2}{*}{ Taxon } & \multirow[t]{2}{*}{ Voucher/culture } & \multicolumn{2}{|c|}{$\begin{array}{l}\text { GenBank accession } \\
\text { Numbers }\end{array}$} & \multirow[t]{2}{*}{ References } \\
\hline & & ITS & LSU & \\
\hline Puccinia kuehnii & 090687 & GU564421 & -- & Glynn et al. (2010) \\
\hline Puccinia lantanae & R190 & -- & EU851144 & Zuluaga et al. (2011) \\
\hline Puccinia lateritia & BPI 910249 & -- & KY764134 & $\begin{array}{l}\text { Demers et al. } \\
\text { (unpublished) }\end{array}$ \\
\hline Puccinia liberta & BRIP 59686 & -- & KX999881 & Marin-Felix et al. (2017) \\
\hline Puccinia linkii & DAOM:242721 & KM851040 & -- & $\begin{array}{l}\text { Mulvey \& Hambleton } \\
\text { (2015) }\end{array}$ \\
\hline uccinia linkii & DAOM:243230 & -- & KM851041 & $\begin{array}{l}\text { Mulvey \& Hambleton } \\
\text { (2015) }\end{array}$ \\
\hline Puccinia malvacearum & AFTOL-ID 1629 & EF561641 & -- & $\begin{array}{l}\text { Matheny \& Hibbett } \\
\text { (unpublished) }\end{array}$ \\
\hline Puccinia malvacearum & U1384 & -- & MG907249 & Aime et al. (2018) \\
\hline $\begin{array}{l}\text { Puccinia } \\
\text { melanocephala }\end{array}$ & PM_NayMex_Xalsco & MG564638 & -- & $\begin{array}{l}\text { Bermudez et al. } \\
\text { (unpublished) }\end{array}$ \\
\hline $\begin{array}{l}\text { Puccinia } \\
\text { melanocephala }\end{array}$ & PM_ColMex_CE_Tecoman & -- & MG564636 & $\begin{array}{l}\text { Bermudez et al. } \\
\text { (unpublished) }\end{array}$ \\
\hline Puccinia menthae & BPI 910255 & KY764141 & -- & $\begin{array}{l}\text { Demers et al. } \\
\text { (unpublished) }\end{array}$ \\
\hline Puccinia menthae & BPI 871110 & & DQ354513 & Aime et al. (2006) \\
\hline Puccinia nakanishikii & BPI 910261 & KY764147 & -- & $\begin{array}{l}\text { Demers et al. } \\
\text { (unpublished) }\end{array}$ \\
\hline Puccinia nakanishikii & -- & $-{ }_{-1}$ & GU058002 & Dixon et al. (2010) \\
\hline Puccinia nepalensis & BA66 & -- & KX014746 & Ali et al. (2017) \\
\hline Puccinia nepalensis & BA651 & KX225481 & -- & Ali et al. 2017) \\
\hline Puccinia nitida & BPI 843472 & -- & KY764148 & $\begin{array}{l}\text { Demers et al. } \\
\text { (unpublished) }\end{array}$ \\
\hline Puccinia oahuensis & BPI087481 & -- & KY575092 & $\begin{array}{l}\text { Demers \& Castlebury } \\
\text { (unpublished) }\end{array}$ \\
\hline Puccinia obscura & KR14322 & -- & FJ669234 & Scholler et al. (2011) \\
\hline Puccinia obscura & -- & AF468042 & -- & Weber et al. (2003) \\
\hline Puccinia ocimi & BPI 910262 & KY764149 & KY764149 & $\begin{array}{l}\text { Demers et al. } \\
\text { (unpublished) }\end{array}$ \\
\hline Puccinia operta & BPI142436 & & KY575095 & $\begin{array}{l}\text { Demers \& Castlebury } \\
\text { (unpublished) }\end{array}$ \\
\hline Puccinia oxalidis & KUS-F27920 & MH325473 & -- & Lee et al. (2019) \\
\hline Puccinia oxalidis & BPI 893310 & -- & KY798348 & $\begin{array}{l}\text { Demers \& Castlebury } \\
\text { (unpublished) }\end{array}$ \\
\hline Puccinia paspali & BPI841180 & -- & KY575098 & $\begin{array}{l}\text { Demers \& Castlebury } \\
\text { (unpublished) }\end{array}$ \\
\hline Puccinia peradeniyae & BPI 089014 & KX190906 & KX190906 & Demers et al. (2017) \\
\hline Puccinia pimpinellae & -- & GU058023 & GU058023 & Dixon et al. (2010) \\
\hline $\begin{array}{l}\text { Puccinia polygoni- } \\
\text { amphibii }\end{array}$ & BPI 893289 & -- & KY798381 & $\begin{array}{l}\text { Demers \& Castlebury } \\
\text { (unpublished) }\end{array}$ \\
\hline Puccinia polysora & -- & HM467909 & GU058024 & Yu et al. (unpublished) \\
\hline Puccinia porri & $22-16$ & KY492366 & -- & $\begin{array}{l}\text { Ristic et al. } \\
\text { (unpublished) }\end{array}$ \\
\hline Puccinia recondita & BPI 910319 & -- & KY798399 & $\begin{array}{l}\text { Demers \& Castlebury } \\
\text { (unpublished) }\end{array}$ \\
\hline Puccinia rufipes & -- & AJ406071 & -- & Virtudazo et al. (2001) \\
\hline Puccinia scirpi & BRIP 61027 & KX999892 & KX999892 & Marin-Felix et al. (2017) \\
\hline Puccinia striiformis & HSZ1834 & GQ457306 & -- & Jin et al. (2010) \\
\hline Puccinia striiformis & HSZ1828 & -- & GQ457304 & Jin et al. (2010) \\
\hline Puccinia substriata & -- & GU058028 & -- & Dixon et al. (2010) \\
\hline
\end{tabular}


Table 1 Continued.

\begin{tabular}{|c|c|c|c|c|}
\hline \multirow[t]{2}{*}{ Taxon } & \multirow[t]{2}{*}{ Voucher/culture } & \multicolumn{2}{|c|}{$\begin{array}{l}\text { GenBank accession } \\
\text { Numbers }\end{array}$} & \multirow[t]{2}{*}{ References } \\
\hline & & ITS & LSU & \\
\hline Puccinia substriata & BPI106260 & & KY575101 & $\begin{array}{l}\text { Demers \& Castlebury } \\
\text { (unpublished) }\end{array}$ \\
\hline Puccinia tanaceti & R221 & HQ201323 & HQ201324 & Alaei (unpublished) \\
\hline Puccinia thlaspeos & -- & L76177 & & Roy et al. (1998) \\
\hline Puccinia thlaspeos & -- & L76183 & & Roy et al. (1998) \\
\hline Puccinia turgida & NA224 & MH144383 & -- & Otálora \& Berndt (2018) \\
\hline Puccinia turgida & NA223 & MH144382 & & Otálora \& Berndt (2018) \\
\hline Puccinia versicolor & U902 & -- & MG907252 & Aime et al. (2018) \\
\hline Puccinia violae & BPI 842321 & DQ354509 & -- & Aime (2006) \\
\hline Puccinia violae & -- & -- & GU058029 & Dixon et al. (2010) \\
\hline Puccinia xanthii & BRIP48819 & EU659694 & -- & Seier et al. (2009) \\
\hline Puccinia xanthii & BRIP 56946 & & KX999896 & Marin-Felix et al. (2017) \\
\hline Pucciniastrum coryli & TSH-R4237 (IBA8641) & AB221419 & -- & Liang et al. (2006) \\
\hline $\begin{array}{l}\text { Ravenelia acaciae- } \\
\text { arabicae }\end{array}$ & PREM61853 & -- & MN072675 & Ebinghaus et al. (2020) \\
\hline $\begin{array}{l}\text { Ravenelia acaciae- } \\
\text { pennatulae }\end{array}$ & U115 & -- & MG907213 & Aime et al. (2018) \\
\hline Ravenelia acaciicola & PREM61861 & -- & MN072683 & Ebinghaus et al. (2020) \\
\hline Ravenelia evansii & PREM61209 & MG945959 & -- & Ebinghaus et al. (2018) \\
\hline Ravenelia evansii & PREM61028 & -- & MG945996 & Ebinghaus et al. (2018) \\
\hline Tranzschelia discolor & U-884 & DQ995341 & -- & Deadman et al. (2007) \\
\hline Tranzschelia discolor & BRIP 57662 & -- & KR994891 & $\begin{array}{l}\text { Doungsa-Ard } \\
\text { (unpublished) }\end{array}$ \\
\hline $\begin{array}{l}\text { Tranzschelia pruni- } \\
\text { spinosae }\end{array}$ & KR-M-0002755 & -- & KX228774 & Scholler et al. (2019) \\
\hline Uromyces aloes & 2020-6-28-0005 & MT136509 & -- & Bily et al. (unpublished) \\
\hline Uromyces aloes & WM 3290 & -- & DQ917740 & Maier et al. (2007) \\
\hline $\begin{array}{l}\text { Uromyces ciceris- } \\
\text { arietini }\end{array}$ & -- & GU058030 & -- & Dixon et al. (2010) \\
\hline $\begin{array}{l}\text { Uromyces ciceris- } \\
\text { arietini }\end{array}$ & & -- & GQ914998 & Stuteville et al. (2010) \\
\hline Uromyces clignyi & DAOM 192217 & HM131364 & -- & Liu \& Hambleton (2013) \\
\hline Uromyces commelinae & JW100 & KF982855 & -- & Kwon \& Kim (2014) \\
\hline Uromyces coronatus & DAOM 32991 & HM131365 & -- & Liu \& Hambleton (2013) \\
\hline Uromyces coronatus & BPI 910293 & & KY764191 & $\begin{array}{l}\text { Demers et al. } \\
\text { (unpublished) }\end{array}$ \\
\hline Uromyces dactylidis & PRC:705 & KM667955 & -- & Hrabetova et al. (2015) \\
\hline Uromyces dactylidis & TUB 14997 & -- & DQ917745 & Maier et al. (2007) \\
\hline Uromyces dolicholi & DAOM 116149 & HQ317563 & HQ317563 & Liu et al. (2015) \\
\hline Uromyces eragrostidis & DAOM 106767 & HQ317561 & -- & Liu et al. (2015) \\
\hline Uromyces eragrostidis & BPI004474 & -- & KY575113 & $\begin{array}{l}\text { Demers \& Castlebury } \\
\text { (unpublished) }\end{array}$ \\
\hline Uromyces euphorbiae & PUR N11621 & KT750329 & -- & $\begin{array}{l}\text { Rosskopf et al. } \\
\text { (unpublished) }\end{array}$ \\
\hline Uromyces euphorbiae & BPI 863673 & -- & KT750330 & $\begin{array}{l}\text { Rosskopf et al. } \\
\text { (unpublished) }\end{array}$ \\
\hline Uromyces geranii & BRIP 60100 & -- & KX999898 & Marin-Felix et al. (2017) \\
\hline $\begin{array}{l}\text { Uromyces hedysari- } \\
\text { obscuri }\end{array}$ & DAOM 189699 & HQ317573 & -- & Liu et al. (2015) \\
\hline $\begin{array}{l}\text { Uromyces hedysari- } \\
\text { obscuri }\end{array}$ & DAOM 223015 & -- & HQ317572 & Liu et al. (2015) \\
\hline $\begin{array}{l}\text { Uromyces lespedezae- } \\
\text { procumbentis }\end{array}$ & BPI 910294 & KY764193 & KY764193 & $\begin{array}{l}\text { Demers et al. } \\
\text { (unpublished) }\end{array}$ \\
\hline
\end{tabular}


Table 1 Continued.

\begin{tabular}{|c|c|c|c|c|}
\hline \multirow[t]{2}{*}{ Taxon } & \multirow[t]{2}{*}{ Voucher/culture } & \multicolumn{2}{|c|}{$\begin{array}{l}\text { GenBank accession } \\
\text { Numbers }\end{array}$} & \multirow[t]{2}{*}{ References } \\
\hline & & ITS & LSU & \\
\hline Uromyces minor & MVAP50000151 & MK045314 & MK045314 & Blomquist (unpublished) \\
\hline Uromyces muscari & DAOM 75626 & HQ317552 & HQ317552 & Liu et al. (2015) \\
\hline Uromyces orientalis & BRIP 60934 & KX999899 & KX999899 & Marin-Felix et al. (2017) \\
\hline Uromyces peglerae & BPI843311 & & KY575116 & $\begin{array}{l}\text { Demers \& Castlebury } \\
\text { (unpublished) }\end{array}$ \\
\hline Uromyces pisi-sativi & -- & DQ521591 & -- & $\begin{array}{l}\text { Sagliocco et al. } \\
\text { (unpublished) }\end{array}$ \\
\hline Uromyces pisi-sativi & BRIP 60151 & -- & KX999900 & Marin-Felix et al. (2017) \\
\hline $\begin{array}{l}\text { Uromyces polygoni- } \\
\text { avicularis }\end{array}$ & DAOM 181565 & & HQ317558 & Liu et al. (2015) \\
\hline Uromyces rumicis & PDD:93529 & KX985763 & -- & $\begin{array}{l}\text { Padamsee \& McKenzie } \\
(2017)\end{array}$ \\
\hline Uromyces rumicis & BPI 910298 & & KY764197 & $\begin{array}{l}\text { Demers et al. } \\
\text { (unpublished) }\end{array}$ \\
\hline $\begin{array}{l}\text { Uromyces setariae- } \\
\text { italicae }\end{array}$ & BPI863744 & KY575068 & & $\begin{array}{l}\text { Demers \& Castlebury } \\
\text { (unpublished) }\end{array}$ \\
\hline $\begin{array}{l}\text { Uromyces setariae- } \\
\text { italicae }\end{array}$ & BPI863750 & -- & KY575069 & $\begin{array}{l}\text { Demers \& Castlebury } \\
\text { (unpublished) }\end{array}$ \\
\hline Uromyces striatus & U-675 & HQ412651 & -- & Deadman et al. (2011) \\
\hline Uromyces striatus & DAOM 240966 & -- & HQ317512 & Liu et al. (2015) \\
\hline Uromyces strobilanthis & BPI 893253 & -- & KY798375 & $\begin{array}{l}\text { Demers \& Castlebury } \\
\text { (unpublished) }\end{array}$ \\
\hline Uromyces tenuicutis & BRIP 60012 & -- & KX999904 & Marin-Felix et al. (2017) \\
\hline Uromyces trifolii & -- & -- & GU936634 & Zuluaga et al. (2011) \\
\hline Uromyces viciae-fabae & -- & AB115665 & -- & Chung et al. (2004) \\
\hline Uromyces viciae-fabae & & -- & AF426199 & Maier et al. (2003) \\
\hline Uromyces vignae & H92019 & AB115731 & -- & Chung et al. (2004) \\
\hline Uromyces vignae & BRIP 60213 & -- & KX999906 & Marin-Felix et al. (2017) \\
\hline Taphrina pruni & CBS 358.35 & MH855700 & MH867219 & Vu et al. 2019) \\
\hline
\end{tabular}

\section{Genus-wise literature of Indian rust fungi}

Angiopsora: Bagchee \& Singh 1960, Bahadur \& Sinha 1967, Ramakrishnan 1950, Ramakrishnan \& Sundaram 1954b, Thirumalachar \& Mundkar 1951, Vaheeduddin 1955. Arthuria: Gokhle \& Patel 1953. Bubakia: Butler \& Bisby 1931, Mundkar 1938, 1943, Ramakrishnan \& Ramakrishnan 1950a. Calidion: Ramakrishnan 1950. Chaconia: Butler \& Bisby 1931, Mishra et al. 1976, Patel et al. 1949, Patil \& Thirumalachar 1971, Patil 1966a, Ramakrishnan 1950, Singh 1966. Ceropsora: Bakshi \& Singh 1960. Cerotelium: Ahmad 1981, Arthur 1917, Bakshi et al. 1972, Butler 1914, Chowdhary 1948, Jain et al. 1966, Joshi \& Vashiist 1959, Nagraj et al. 1971, Patil \& Thirumalachar 1971, Patil 1966a, Pawar \& Kulkarni 1973, Payak 1949, Ramachar et al. 1978, Ramakrishnan 1952, Rangaswamy et al. 1968, Sathe 1972b, Sydow \& Mitter 1935, Thite \& Patil 1970, Vasudeva 1962, Venkatakrishaiya 1958, Wakhloo 1962, Yadav 1963a, Yadav \& Thirumalachar 1955. Chrysocelis: Ramakrishnan \& Ramakrishnan 1949. Chrysomyxa: Bakshi \& Singh 1972, Barclay 1890a, Butler 1906, 1910, Dietal 1890, Puri 1955, Sydow \& Butler 1901, Ulbitch 1938. Coleosporium: Anonymous 1950, Arthur 1934, Bagchee 1950b, Bakshi et al. 1972, Barclay 1890b, Berkeley 1856, Butler \& Bisby 1931, Cummins 1943, Dewan \& Kar 1974, Fleming 1874, Goswami \& Singh 1973, Hafeezkhan 1928b, Kamal et al. 1979, Mitter \& Tandon 1932a, b, Patil \& Thirumalachar 1971, Patil 1966a, Prasada 1951, Puri 1955, Sanwal 1951b, Sydow 1922, Sydow \& Mitter 1933, Sydow et al. 1937, Sydow \& Butler 1901, Sydow et al. 1907, Sydow et al. 1912, Wani \& Thirumalachar 1969, Yadav 1964a, Yadav \& Thirumalachar 1955. Corbulopsora: Thirumalachar 1947. Cronartium: Anonymous 1950, Bagchee 1933, 1950a, b, c, Bakshi et al. 1972, 
Butler \& Bisby 1931, Goswami \& Singh 1973, Hafeezkhan 1928a, Puri 1955. Crossopsora: Chavan \& Bakare 1974, Malviya \& Jain 1981, Mundkar \& Thirumalachar 1945, Mundkar \& Thirumalachar 1952, Patil \& Thirumalachar 1971, Patil 1966a, Ramakrishnan \& Soumini 1946a, Sunderam 1961, Sydow et al. 1912, Yadav 1963b. Cystopsora: Ramakrishnan \& Sundaram 1952a, Rangaswamy et al. 1968, Sharma 1977. Dasturella: Butler \& Bisby 1931, Mishra \& Nema 1976, Mundkar \& Kheshwala 1943, Nema \& Mishra 1965, Patel et al. 1949, 1951b, Patil \& Thirumalachar 1971, Patil 1966a, Rangaswamy et al. 1968, Sathe 1965a, Thirumalachar et al. 1956, Thirumalachar \& Gopalkrishan 1947, Yadav 1964a. Didymopsorella: Rangaswamy et al. 1968, Thirumalachar 1950a, 1951, Thirumalachar \& Mundkar 1950. Diorchidium: Sydow et al. 1907, Yadav 1953. Elateraecium: Thirumalachar et al. 1966. Endophyllum: Arthur 1934, Cummins 1943, Gokhle et al. 1955, Singh \& Jalan 1965, Nagraj et al. 1971, Patil 1966a, Thirumalachar \& Govindu 1954, Thirumalachar \& Narsimhan 1950b. Gambleola: Butler 1906, Sydow \& Butler 1901, Sydow et al. 1907. Goplana: Ramakrishnan \& Ramakrishnan 1949. Gymnopuccinia: Ramakrishnan 1951c, Thite \& Patil 1970. Gymnosporangium: Arthur 1934, Arthur \& Cummins 1936a, Barclay 1890d, Butler 1906, Cummins 1943, Sydow 1938, Sydow \& Butler 1901. Hamaspora: Goswami \& Singh 1973, Massee 1892, Ramakrishnan \& Sundaram 1954a. Hapalophragmium: Jain et al. 1966, Mishra 1969, Ramakrishnan \& Ramakrishnan 1948a, Thirumalachar 1950c. Hemileia: Ananth \& Chokanna 1961, Cooke 1876b, Krishnamurthy \& Rangaswamy 1947, Massee 1906, Nagraj et al. 1971, Parndekar 1964, Patil \& Thirumalachar 1971, Patil 1977, 1966a, Ramakrishnan 1957b, Ramakrishnan \& Soumini 1946b, Rangaswamy et al. 1968, Sydow \& Mitter 1933, Sydow \& Butler 1901, Thirumalachar 1947, Thirumalachar 1950b, Thirumalachar \& Narsimhan 1947, Thite \& Patil 1970, Yadav 1963b. Hyalopsora: Anonymous 1936, Arthur 1934, Chona \& Munjal 1955, Munjal \& Kapoor 1961, Sydow 1938. Kernella: Thirumalachar \& Mundkar 1949. Kernkampella: Rajendren 1970, Bhagyanarayana \& Ramachar 1985. Kuehneola: Chavan 1975, Malviya \& Jain 1981, Patil 1966a, Thite \& Patil 1970, Yadav 1963b, Bhagyanarayana \& Rao 1995, Hosagoudar 1985. Kweilingia: Bakshi et al. 1972, Gautam \& Avasthi 2018. Leucotelium: Sydow 1939, Sydow et al. 1912, Nema \& Mishra 1965. Macabuna: Kamal et al. 1979. Maravalia: Ahmad 1981, Arthur \& Cummins 1936b, Butler \& Bisby 1931, Patil \& Thirumalachar 1971, Patil 1966a, Thirumalachar 1949a, Yadav \& Thirumalachar 1955. Masseeëlla: Joshi \& Vashiist 1959, Mundkar \& Thirumalachar 1952, Patel et al. 1949, Patil 1966a, Patwardhan 1964, Ramakrishnan 1957a, Sharma 1975, Thirumalachar 1943b, c. Melampsora: Agarwal et al. 1959, Ajrekar 1912, Bagchee 1950b, Bagchee \& Singh 1960, Bakshi \& Singh 1961, Barclay 1891, Butler 1905, 1918, Butler \& Bisby 1931, 1960, Chavan \& Bakare 1977, Cooke 1876b, Cummins 1943, Jain et al. 1966, Kala \& Gaur 1983a, Kaul 1959a, b, Kern \& Thurston 1944, Lele 1952, Manocharachary et al. 1976, Mcrae 1917, Mishra 1969, 1963a, Mishra \& Prasad 1966, Pandotra 1966, Pandotra \& Ganguly 1964b, Parndekar 1964, Patil \& Thirumalachar 1971, Patil 1966a, Payak 1949, Puri 1955, Ramachar \& Bhagyanarayana 1977a, b, Ramakrishnan \& Ramakrishnan 1949, Rangaswamy et al. 1968, Ravindra Nath \& Narahari Reddy 1964, Srivastava 1982, Sydow \& Butler 1901, Sydow et al. 1907, Sydow et al. 1912, Thirumalachar 1941b, Vasudeva 1948, 1949, 1950a, b. Melampsoridium: Bakshi et al. 1972, Narayan \& Kamal 1985, Patil 1966a, Sathe 1966a, Singh \& Pandey 1972. Milesina: Anonymous 1959a, Bagchee \& Singh 1960. Monosporidium: Barclay 1890c, Parndekar 1964, Patil 1966a, Sydow et al. 1912, Gokhle \& Patel 1951. Neophysopella: Mundkar 1943, Vaheeduddin 1955, Bagchee \& Singh 1960. Nyssopsora: Mundkar \& Thirumalachar 1952, Nagachan \& Goswami 1985, Rangaswamy et al. 1968, Roy 1948. Ochropsora: Arthur \& Cummins 1936a. Olivea: Bakshi et al. 1972, Butler \& Bisby 1931, Pandotra \& Ganguly 1964b, Patil 1966a. Peridermium: Anonymous 1950, Bakshi \& Singh 1972, Barclay 1890b, Butler \& Bisby 1931, Champion 1922, Cooke 1878b, Hafeezkhan 1928a, Mitter \& Tandon 1932a, Puri 1955, Sydow \& Butler 1901, Berkeley 1856, Cooke 1877. Peridiopsora: Sathe 1969b. Phakopsora: Bahekar 1966, Butler 1912, Butler \& Bisby 1931, Dietal 1890, Jhooty et al. 1977, Kala \& Gaur 1983a, Mundkar 1943, Nagraj et al. 1971, Patel et al. 1985, Patil \& Thirumalachar 1971, Patil 1977, Ramachar et al. 1978, Ramakrishnan \& Subramanian 1952, Ramakrishnan 1951c, Ramakrishnan 1952, 1955a, 1956, Ramakrishnan \& Ramakrishnan 1950a, Ramakrishnan et al.1952, Ramakrishnan \& Sundaram 1954b, 1955a, b, Rangaswamy et al. 1968, Sathe 1972a, Sharma \& Jain 
1981, Sydow 1938, Sydow et al. 1907, Sydow et al. 1912, Thirumalachar 1947, Uppal et al. 1935, Yadav 1963a, 1964a, Yadav \& Thirumalachar 1955. Phragmidiella: Patel et al. 1949, Patil 1966a, Thirumalachar \& Mundkar 1949. Phragmidium: Barclay 1890b, Barclay 1891, Bhattacharya \& Baruah 1953, Cooke 1878a, b, Goswami \& Singh 1973, Kala \& Gaur 1983a, Mitter \& Tandon 1932a, b, Mundkar 1938, Pandotra \& Ganguly 1964b, Ramakrishnan \& Sundaram 1953a, Sydow 1938, Sydow \& Mitter 1935, Sydow et al. 1907, Sydow et al. 1911a, b. Physopella: Agarwal et al. 1964, Khanna \& Chandra 1975, Malviya \& Jain 1981, Patil \& Thirumalachar 1971, Ramachar 1966, Ramachar \& Bhagyanarayana 1976, Sathe 1965d, Subramaniam \& Ramakrishnan 1956. Pileolaria: Sydow 1938, Gautam \& Avasthi 2017c. Prospodium: Bagyanarayana \& Ravinder 1995, Bagyanarayana et al. 1998. Puccinia: Agarwal et al. 1959, 1981, Ahmad 1977, Ahmad \& Singh 1969, Ahmad et al. 1969, Anonymous 1950, Arthur 1934, Arthur \& Cummins 1936a, Bagchee \& Singh 1960, Balasubramanian 1973, Barclay 1890a, b, e, f, 1891, Berkeley 1856, Butler 1905, 1918, Butler \& Bisby 1931, 1960, Butler \& Hayman 1906, Chavan 1975, Chavan \& Bakare 1974, Chavan \& Bakare 1977, Chavan \& Patil 1972, Chona et al. 1958, Chona \& Munjal 1950, 1955, 1956, Chona et al. 1956, Chowdhary 1948, Cooke 1876a, b, Cooke 1878a, Cummins 1943, Cummins 1953, Dalela 1956, Dalela \& Sinha 1964, Damle 1943, Darr \& Shah 1980, Deoraj 1980, Dietal 1890, Dube 1958, Fleming 1874, Ganguly \& Pandotra 1962, 1963, Gautam \& Avasthi 2016b, Gautam \& Avasthi 2017a, Gopinathnair 1972, Goswami 1974, Goswami \& Singh 1973, Goyal et al. 1971, Gupta 1977, Gupta \& Shukla 1955, Jadhav \& Somani 1978a, b, Jain et al. 1966, Joshi \& Lele 1984, Joshi \& Merchand 1963, Joshi \& Payak 1963, Joshi 1958, Joshi \& Vashiist 1959, Kala \& Gaur 1983a, b, Kamal et al. 1979, Kanaujia 1978, Kanaujia \& Kishore 1981, Kanadswamy \& Vijyalakshmi 1959, Kaul 1959b, Khanna 1961, Khosla et al. 1975, Khulbe \& Verma 1978, Kolte \& Awasthi 1979, Konger \& Baruah 1958, Kumar et al. 1975, Malviya \& Jain 1981, Mcrae 1917, Mehta 1934, Mishra et al. 1964, 1965, 1968, Mishra 1969, Mishra \& Nema 1976, Mishra et al. 1976, Mishra \& Mishra 1975, Mishra 1963b, Mishra \& Lele 1963, Mishra \& Sharma 1963, 1964, Mishra et al. 1975, Mitter \& Tandon 1930, 1932a, b, 1937, Mundkar 1938, Mundkar \& Ahmad 1946, Mundkar \& Thirumalachar 1952, Mutkekar et al. 1968, Munshi 1976, Nema \& Agarwal 1960, Nema \& Mishra 1965, Padwick 1945a, b, Padwick \& Khan 1944, Pandotra 1966, Pandotra \& Ganguly 1962, Pandotra \& Ganguly 1964a, b, Pandotra \& Sastry 1969a, b, Parndekar 1964, Patel et al. 1949, 1950, Patil \& Thirumalachar 1964, Patil \& Thirumalachar 1971, Patil 1966a, Patil \& Date 1980, Payak 1949, 1965, Payak \& Khanna 1970, Payak \& Mishra 1963, Payak \& Renfro 1966, Prasad 1948, Prasada 1951, Rebenhorst 1878, Sharma \& Shankar 1979, Rajendran 1966, Ramachar 1965, Ramachar et al. 1978, Ramachar \& Bhagyanarayana 1976, Ramachar \& Cummmins 1965, Ramakrishna \& Subbayya 1973, Ramakrishnan 1950, Ramakrishnan 1952, 1955b, 1956, Ramakrishnan \& Narasimhalu 1941, Ramakrishnan \& Ramakrishnan 1946, Ramakrishnan \& Ramakrishnan 1947, 1948a, b, Ramakrishnan \& Ramakrishnan 1949, 1950b, c, Ramakrishnan et al. 1952a, b, Ramakrishnan \& Sundaram 1952b, 1953b, 1954a, b, 1955a, 1956a, b, Rangaswamy et al. 1968, Roy 1964, 1968, Roy \& Gupta 1959, Sachan et al. 1980, Sahni \& Chona 1965, Salam \& Ramachar 1955, Sathe 1965b, 1969a, 1971, Shanmugam et al. 1972, Sharma \& Mukerji 1972, Sharma 1975, Sharma et al. 1979, Sharma \& Singh 1964, Sharma 1957, Sharma et al. 1973, Shinde \& More 1975, Shukla \& Singh 1976, Siddiqui 1971, 1972, 1973, Singh \& Kamal 1985, Singh \& Sharma 1977, Singh 1962, Mishra et al. 1965, Mishra 1969, Sinha \& Kapooria 1966, Sohi et al. 1967, Somani 1979, Soumini 1949, Srivastava 1979a, c, 1980, 1982, Subramaniam \& Ramakrishnan 1956, Sunderam 1956, 1963, Sunderam et al. 1966, Sydow 1913, 1914, 1922, 1938, 1939, Sydow \& Mitter 1935, Sydow et al. 1937, Sydow \& Butler 1901, Sydow et al. 1907, Sydow et al. 1911a, b, Sydow et al. 1912, Thirumalachar 1941a, 1945, Thirumalachar 1947, 1949a, b, Thirumalachar \& Mundkar 1951, Thirumalachar et al. 1943, Tilak \& Rao 1968, Ulbitch 1938, Unni \& Philip 1974, Uppal et al. 1935, Vasudeva 1948, 1949, 1950a, b, 1957, 1958, Wakhloo 1962, Yadav 1953, 1963b, 1964a, Yadav \& Yadav 1965, Yadav et al. 1975. Pucciniastrum: Bakshi et al. 1972, Patil 1966a. Pucciniosira: Arthur 1934, Cummins 1943. Pucciniostele: Barclay 1891, Sydow et al. 1912. Ramakrishnania: Ramachar \& Bhagyanarayana 1979. Ravenelia: Berkeley 1839, Butler \& Bisby 1931, Chavan \& Bakare 1974, Cooke 1880a, b, Jain et al. 1966, Kapoor \& Agarwal 1972, 1974, Mishra 1969, Mishra et al. 1976, 
Mundkar \& Prasad 1938, Narasimhan \& Thirumalachar 1961, Patil \& Thirumalachar 1971, Patil 1977, Patil \& Thite 1978, Patil 1966a, b, Patil \& Date 1977, Pavgi \& Singh 1969, Petch 1912, Ramakrishnan 1952, 1957a, Ramakrishnan \& Ramakrishnan 1948b, Ramakrishnan \& Sundaram 1952b, Rangaswamy et al. 1968, Roy 1964, Salam \& Ramachar 1956, Sanwal 1951a, Sathe 1965c, Siddiqui 1957, Sydow 1913, Sydow \& Mitter 1933, Sydow \& Butler 1901, Sydow et al. 1907, Sydow et al. 1912, Tyagi \& Prasad 1972, 1978, Yadav 1963b, Yadav \& Thirumalachar 1955. Roestelia: Barclay 1891, Arthur \& Cummins 1936a, Mitter \& Tandon 1932a, 1937, Sydow et al. 1907. Scopella: Butler \& Bisby 1931, Cummins1950, Mundkar \& Thirumalachar 1952, Narayan \& Kamal 1985, Parndekar 1964, Patil \& Thirumalachar 1971, Payak 1949, Solanki et al. 1985, Thirumalachar 1950c, Thirumalachar \& Mundkar 1951. Skierka: Chavan 1968, Gautam \& Avasthi 2017c. Sphaerophragmium: Cooke 1880a. Stakmania: Sathe 1966c. Trachyspora: Arthur 1934, Padwick \& Merh 1943. Tranzschelia: Patel et al. 1949, Waraitch \& Khatri 1976, 1977. Trochodium: Gharse 1944, Patil \& Thirumalachar 1971, Patil 1966a, Thirumalachar 1942a. Uredinopsis: Anonymous 1936, Munjal \& Kapoor 1961, Patil 1966a, Puri 1955, Ramakrishnan \& Ramakrishnan 1950b. Uredopeltis: Ramachar et al. 1978, Sathe 1965e. Uromyces: Agarwal et al. 1959, Ahmad 1941, Ajrekar 1912, Ajrekar \& Tonapy 1923, Anonymous 1950, 1954, Arthur 1934, Arthur \& Cummins 1936a, Asthana 1952, Bahadur \& Sinha 1967, Barclay 1890a, 1891, Basuchaudhary \& Singh 1971, Behera \& Mukerji 1974, Butler \& Bisby 1931, 1960, Chaudhari 1958, Chavan 1975, Chavan \& Bakare 1973a, b, Chavan \& Bakare 1974, Chavan \& Bakare 1977, Cooke 1876a, Cummins 1943, Dietal 1890, Dube 1958, Dube et al. 1979, Dublish \& Singh 1977, Gautam \& Avasthi 2017b, Gerdemann \& Bakshi 1976, Jain et al. 1966, Joshi \& Reddy 1958, Joshi \& Reddy 1959, Joshi 1958, Kapooria \& Sinha 1966, Manocharachary 1975, Mathur 1967, Mathur \& Singh 1964, Mishra 1969, Mishra \& Khare 1969, Mishra \& Nema 1976, Mishra et al. 1976, Mitter \& Tandon 1930, 1932b, More \& Moniz 1964, Nagachan \& Verma 1984, Narasimhan \& Thirumalachar 1964, Nema \& Agarwal 1960, Nema \& Mishra 1965, Padwick \& Khan 1944, Padwick \& Merh 1943, Pandotra 1966, Pandotra \& Sastry 1969a, Parndekar 1964, Patel et al. 1949, Patil \& Thirumalachar 1968, Patil \& Thirumalachar 1962, Patil \& Thirumalachar 1971, Patil 1966a, Patil \& Date 1980, Pavgi \& Upadhyay 1966, Payak 1949, 1953, 1962, Ponappa 1969, Prasad et al. 1962, Rebenhorst 1878, Ramachar et al. 1978, Ramakrishnan \& Subramanian 1952, Ramakrishnan 1951b, Ramakrishnan 1952, Ramakrishnan \& Ramakrishnan 1948b, Ramakrishnan \& Rangaswamy 1948, Ramakrishnan \& Shrinivasan 1950, Ramakrishnan et al. 1952a, b, Ramakrishnan \& Sundaram 1955a, Rangaswamy et al. 1968, Rolla \& Addala 1963, Sachan et al. 1980, Saini \& Chand 1984, Saksena 1956, 1930, Sokhi \& Sohi 1976, Srivastava 1979b, Sunderam 1964, Sydow 1913, 1922, 1938, Sydow \& Mitter 1935, Sydow et al. 1937, Sydow \& Butler 1901, Sydow et al. 1907, Sydow et al. 1912, Thite \& Patil 1970, Ulbitch 1938, Uppal et al. 1935, Vasudeva 1948, 1949, 1950a, b, Yadav 1963b, Yadav \& Thirumalachar 1955. Xenostele: Ramakrishnan 1951a. Zaghouania: Butler \& Bisby 1931.

\section{Pucciniales genera incertae sedis}

Aecidium: Ajrekar 1936, Arthur \& Cummins 1936a, Bagchee \& Singh 1960, Barclay 1890a, b, 1891, Bhagat \& Kelkar 1974, Butler \& Bisby 1931, 1960, Chavan \& Bakare 1973a, b, 1974, Chavan \& Patil 1972, Cooke 1878a, Cummins 1943, Goswami 1972, Goswami \& Bhattacharjee 1973, Gupta \& Gupta1985, Hennings 1900, Hosagaudar 1984, Kamal \& Singh 1981, Kamal et al. 1979, Mishra et al. 1976, Mitter \& Tandon 1932a, b, Mundkar 1938, Mundkar \& Thirumalachar 1952, Pandotra \& Ganguly 1964b, Pandotra \& Sastry 1969b, Patel et al. 1949, Patil \& Thirumalachar 1971, Prasad et al. 1962, Ramachar 1956, Ramakrishnan 1959, 1960, Ramakrishnan \& Ramakrishnan 1946, 1948a, 1949, Ramakrishnan et al. 1952a, b, Ramakrishnan \& Sundaram 1953a, b, Rangaswamy et al. 1968, Salam \& Ramachar 1955, 1956, Sathe 1965c, 1966b, Singh \& Kamal 1985, Sunderam \& Rao 1950, Sydow 1913, Sydow 1914, Sydow \& Mitter 1933, Sydow \& Mitter 1935, Sydow \& Sydow 1904a, 1911, 1912a, b, Sydow 1921, Sydow \& Sydow 1917, Sydow \& Butler 1901, Sydow et al. 1907, Sydow et al. 1912, Thirumalachar 1947, Thirumalachar 1950c, Thirumalachar \& Narsimhan 1950a, Thite \& Patil 1970, Ulbitch 1938, Uppal et al. 1935, Yadav 19563b, Yadav \& Saran 1985. Phragmotelium: Thirumalachar 1942b, Thirumalachar et al. 1943. Tunicopsora Suj. Singh \& P.C. 
Pandey: Bakshi et al. 1972. Uraecium: Ramakrishnan 1965, Ramakrishnan \& Ramakrishnan 1948 b. Uredo: Anonymous 1959b, Bagchee \& Singh 1960, Barclay 1890b, 1891, Berkeley 1839, Butler \& Bisby 1931, 1960, Chavan 1975, Chavan \& Bakare 1973a, b, 1974, Chavan \& Bhambure 1975, Cooke 1876b, 1879, Kamal et al. 1979, Kanaujia \& Kishore 1981, Kapoor \& Agarwal 1974, Laundon \& Ponappa 1966, Nagraj et al. 1971, Padwick 1945a, b, Padwick \& Khan 1944, Pandotra 1966, Patel et al. 1951a, Patil 1977, Patil \& Thite 1978, Rahalkar 1977, Ramachar et al. 1978, Ramakrishnan 1951a, 1952, 1956, 1957a, Ramakrishnan \& Ramakrishnan 1948a, Ramakrishnan \& Ramakrishnan 1949, Ramakrishnan et al. 1952a, Ramakrishnan \& Sundaram 1953b, Rangaswamy et al. 1968, Sathe \& Rahalkar 1976, Sunderam 1961, Sydow \& Mitter 1935, Sydow et al. 1937, Sydow \& Sydow 1904b, 1924, Sydow \& Butler 1901, Sydow et al. 1912, Thirumalachar 1947, Thirumalachar 1950c, Vaheeduddin 1955, Yadav 1964b.

\section{Results}

Accepted taxa of rust fungi of Basidiomycota up to genus are summarized in Table 1 while, the species of each genus are mentioned in detailed outline of the fungi reported from India. The rust fungi of India comprised of 640 species and 69 genera belonging to 16 families. Highest numbers of species were reported in Pucciniaceae (393) followed by Raveneliaceae (61), Phakopsoraceae (50), Coleosporiaceae (32), Phragmidiaceae (27), Pucciniastraceae (19), Melampsoraceae (18), Crossopsoraceae (14), Zaghouaniaceae (13), Gymnosporangiaceae (7), Milesinaceae (5), Skierkaceae (3), Tranzscheliaceae (3), Pileolariaceae (2), Ochropsoraceae (1), Sphaerophragmiaceae (1). Similarly, when comparing the rust genera, highest number of species of rust fungi was found Puccinia (279), followed by Uromyces (89), Ravenelia (33), Phakospora (25), Coleosporium (19), Phragmidium (18), Melampsora (17) and Maravalia (11). Many taxa of rust fungi with uncertain taxonomic position are placed in incertae sedis. Similarly, the generic names have been transferred to new genera, but either their types or records from India still need to be revised. Such genera and their species are discussed in notes of Indian rust genera section of this manuscript.

\section{Outline of rust fungi reported from India}

The information presented in the outline is arranged as phylum followed by subphylum, class, order, family, genus and species.

\section{Taxonomy}

Basidiomycota R.T. Moore

Pucciniomycotina R. Bauer, Begerow, J.P. Samp., M. Weiss \& Oberw.

Pucciniomycetes R. Bauer, Begerow, J.P. Samp., M. Weiss \& Oberw.

Pucciniales Clem. \& Shear

1. Coleosporiaceae Dietel, In: Engler \& Prantl, Nat. Pflanzenfam., Teil. I (Leipzig) 1: 548. 1900. emend. Aime \& McTaggart

Coleosporium Lév. (19)

Coleosporium asterum (Dietel) Syd. \& P. Syd.

Coleosporium barclayense Bagchee

Coleosporium bletiae Dietel

Coleosporium campanulae (Pers.) Tul.

Coleosporium clematidis Barclay

Coleosporium datiscae Tranzschel

Coleosporium inulae Rabenh.

Coleosporium ipomoeae (Schwein.) Burrill

Coleosporium himalayense Durrieu

Coleosporium leptodermidis (Barclay) P. Syd. \& Syd. 
Coleosporium mitteri Syd.

Coleosporium myriactidis Syd.

Coleosporium oldenlandiae E.J. Butler

Coleosporium perillae $\mathrm{P}$. Syd.

Coleosporium plectranthi Barclay

Coleosporium satyrii Mundk. \& Thirum.

Coleosporium senecionis (Pers.) Fr.

Coleosporium sidae Sanwal

Coleosporium xanthoxyli Dietel \& P. Syd. (1898)

Chrysomyxa Unger (7)

Chrysomyxa deformans (Dietel) Jacz.

Chrysomyxa dietelii Syd. \& P. Syd.

Chrysomyxa himalensis Barclay

Chrysomyxa piceae Barclay

Chrysomyxa pirolae (DC.) Rostr.

Chrysomyxa vitis E.J. Butler

Chrysomyxa himalayensis Singh, Khan \& Mishra

Cronartium Fr. (4)

Cronartium fici T.S. Ramakr. \& K. Ramakr.

Cronartium himalayense Bagchee

Cronartium quercuum (Berk.) Miyabe ex Shirai

Cronartium ribicola J.C. Fisch.

Goplana Racib. (1)

Goplana indica T.S. Ramakr. \& K. Ramakr.

Stakmania Kamat \& Sathe (1)

Stakmania indica Kamat \& Sathe

2. Crossopsoraceae Aime \& McTaggart, In: Fungal Systematics and Evolution 7: 21-47. 2020

Angiopsora Mains (2)

Angiopsora cyrtococci T.S. Ramakr. \& Sundaram

Angiopsora apoda (Har. \& Pat.) Aime \& McTaggart

Crossopsora Syd. \& P. Syd. (3)

Crossopsora premnae (Petch) Syd. \& P. Syd.

Crossopsora premnae-tomentosae T.S. Ramakr. \& Soumini

Crossopsora symphorematis Sundaram

Crossopsora ziziphi (Syd., P. Syd. \& E.J. Butler) Syd. \& P. Syd.

Dasturella Mundk. \& Khesw. (3)

Dasturella bambusina Mundk. \& Khesw.

Dasturella boswelliae Patel, Payak \& N.B. Kulk.

Dasturella oxytenantherae Sathe

Kweilingia Teng (2)

Kweilingia bagchii (Suj. Singh \& P.C. Pandey) Buriticá

Kweilingia divina (Syd.) Buriticá

Neophysopella Jing X. Ji \& Kakish. (3)

Neophysopella ampelopsidis (Dietel \& P. Syd.) Jing X. Ji \& Kakish.

Neophysopella meliosmae (Kusano) Jing X. Ji \& Kakish.

Neophysopella meliosmae-myrianthae (Henn. \& Shirai) Jing X. Ji \& Kakish.

Physopella (2)

Physopella artocarpi (Berk. \& Broome) Arthur

Physopella vernoniae (T.S. Ramakr.) Ramachar \& Bhagyan. 
3. Gymnosporangiaceae P. Zhou \& L. Cai, Persoonia 45: 79. 2020. emend. Aime \& McTaggart Peridiopsora Kamat \& Sathe (1)

Peridiopsora adelocaryi Kamat \& Sathe

Gymnosporangium R. Hedw. ex DC. (3)

Gymnosporangium clavariiforme (Wulfen) DC.

Gymnosporangium confusum Plowr.

Gymnosporangium cunninghamianum Barclay

Roestelia Rebent. (3)

Roestelia distorta (Arthur \& Cummins) F. Kern

Roestelia cunninghamianum (Barclay) F. Kern

Roestelia patula (Syd. \& P. Syd.) F. Kern

4. Melampsoraceae Dietel, in Engler \& Prantl, Nat. Pflanzenfam., Teil. I (Leipzig) 1: 38. 1897

Ceropsora B.K. Bakshi \& Suj. Singh (1)

Ceropsora piceae (Barclay) B.K. Bakshi \& Suj. Singh

Melampsora Castagne (17)

Melampsora caprearum Thüm.

Melampsora ciliata Barclay

Melampsora damnosa (Sacc.) Lind.

Melampsora epitea Thüm.

Melampsora eucalypti Rabenh.

Melampsora euphorbiae (Ficinus \& C. Schub.) Castagne

Melampsora euphorbiae-geniculatae F. Kern \& Thurst.

Melampsora geniculatae Ramachar \& Bhagyan.

Melampsora hypericorum (DC.) J. Schröt.

Melampsora lini (Ehrenb.) Lév.

Melampsora mundkurii Thirum.

Melampsora oblonga Bagchee

Melampsora populnea (Pers.) P. Karst.

Melampsora salicis-albae Kleb.

Melampsora salicis-wallichianae Ulbr.

Melampsora sancti-johannis Barclay

Melampsora stereospermi T.S. Ramakr. \& K. Ramakr.

Melampsora yoshinagai Henn.

Melampsora caprearum Thüm.

5. Milesinaceae Aime \& McTaggart, In: Fungal Systematics and Evolution 7: 21-47. 2020

Milesina Magnus (3)

Milesina coniogrammes Hirats. f.

Milesina exigua Faull

Milesina polypodii (F.B. White) Aime \& Rossman

Uredinopsis Magnus (2)

Uredinopsis macrosperma (Cooke) Magnus

Uredinopsis syngrammes Munjal \& J.N. Kapoor

6. Ochropsoraceae (Arthur) Aime \& McTaggart, In: Fungal Systematics and Evolution 7: 21-47. 2020

Ochropsora Dietel (1)

Ochropsora ariae (Fuckel) Ramsb.

7. Phakopsoraceae Cummins \& Y. Hirats., Illustr. Gen. Rust Fungi, rev. Edn (St. Paul): 13. 1983. emend. Aime \& McTaggart 
Arthuria Jackson (2)

Arthuria glochidii Gokhale, Patel \& Thirum.

Arthuria tylophorae T.S. Ramakr.

Bubakia Arthur (1)

Bubakia indica T.S. Ramakr. \& K. Ramakr.

Cerotelium Arthur (7)

Cerotelium bauhiniae Thirum. \& Yadav

Cerotelium fici (Castagne) Arthur

Cerotelium kirganeliae Thirum. \& Yadav

Cerotelium peregrinum (P. Syd. \& Syd. \& E.J. Butler) Arthur

Cerotelium terminaliae-paniculatae Nag Raj, Govindu \& Thirum.

Cerotelium trichosanthis (Petch) Nag Raj, Govindu \& Thirum.

Cerotelium wagateae Thirum. \& Gopalkr.

Macabuna Buriticá \& J.F. Hennen (1)

Macabuna ziziphi (Pat.) Buriticá \& J.F. Hennen

Monosporidium Barclay (3)

Monosporidium andrachnes Barclay ex Sacc.

Monosporidium euphorbiae Barclay ex Sacc.

Monosporidium pavettae (Gokhale \& Patel) Buriticá

Masseeella Dietel (6)

Masseeella breyniae Thirum.

Masseeella capparis (Hobson bis ex Cooke) Dietel

Masseeella flueggeae Syd.

Masseeella narasimhanii Thirum.

Masseeella putranjivae T.S. Ramakr.

Masseeella terminaliae Patw.

Phakopsora Dietel (25)

Phakopsora apludae M.S. Patil

Phakopsora artemisiae Hirats. f.

Phakopsora caseariae Yadav

Phakopsora chorisandrae T.S. Ramakr. \& G.S. Reddy

Phakopsora cingens (Syd. \& P. Syd.) Hirats.

Phakopsora cronartiiformis Dietel

Phakopsora desmium (Berk. \& Broome) Cummins

Phakopsora elephantopodis Hirats

Phakopsora elettariae (Racib.) Cummins

Phakopsora erythrinae Gäum.

Phakopsora fici-elasticae T.S. Ramakr.

Phakopsora formosana Syd. \& P. Syd.

Phakopsora incompleta (Syd. \& P. Syd.) Cummins

Phakopsora kirganeliae T.S. Ramakr. \& K. Ramakr.

Phakopsora mangalorica T.S. Ramakr. \& Sundaram

Phakopsora meibomiae (Arthur) Arthur

Phakopsora pachyrhizi Syd. \& P. Syd.

Phakopsora odinae Mundk.

Phakopsora pachyrhizi Syd. \& P. Syd.

Phakopsora parasnathii Yadav \& Thirum.

Phakopsora phyllanthi Dietel

Phakopsora punctiformis (Barclay \& Dietel) Dietel

Phakopsora sterculiae Nag Raj, Govindu \& Thirum.

Phakopsora zingiberis T.S. Ramakr

Phakopsora ziziphi-vulgaris Dietel 
Phragmidiella Henn. (3)

Phragmidiella aliena (Syd., P. Syd. \& E.J. Butler) Buriticá \& J.F. Hennen

Phragmidiella heterophragmatis (Mundk. \& Thirum.) Thirum. \& Mundk.

Phragmidiella holwayi (H.S. Jacks.) Buriticá

Pucciniostele Tranzschel \& K.L. Kom. (1)

Pucciniostele clarkiana (Barclay) Tranzschel \& K.L. Kom.

Uredopeltis Henn. (1)

Uredopeltis chevalieri J. Walker \& R.G. Shivas

8. Phragmidiaceae Corda Icon. fung. (Prague) 1: 6. 1837

Hamaspora Körn. (2)

Hamaspora longissima (Thüm.) Körn.

Hamaspora rubi-sieboldii (Kawagoe) Dietel

Kuehneola Magnus (6)

Kuehneola grewiae (Mundk. \& Thirum.) Thirum.

Kuehneola loeseneriana (Henn.) H.S. Jacks. \& Holw.

Kuehneola flacourtiae (Mundk. \& Thirum.) Thirum.

Kuehneola ramacharii Bagyan. \& K.N. Rao

Kuehneola spondiadis Hosag.

Kuehneola ziziphi (T.S. Ramakr. \& Subram.) Thirum.

Phragmidium Link (18)

Phragmidium assamense Syd. \& P. Syd.

Phragmidium barclayi Dietel

Phragmidium brevipedicellatum Hirats. f.

Phragmidium bulbosum (Fr.) Schltdl.

Phragmidium butleri Syd. \& P. Syd.

Phragmidium egenulum Syd., P. Syd. \& E.J. Butler

Phragmidium fragariae (Rabenh.) Ces.

Phragmidium kamtschatkae (H.W. Anderson) Arthur \& Cummins

Phragmidium mucronatum (Pers.) Schltdl.

Phragmidium orientale Syd. \& P. Syd.

Phragmidium potentillae (Pers.) P. Karst.

Phragmidium rosae-moschatae Dietel

Phragmidium incompletum Barclay

Phragmidium laceianum Barclay

Phragmidium malvacearum Bert.

Phragmidium nepalense Barclay

Phragmidium octoloculare Barclay

Phragmidium quinqueloculare Barclay

Trachyspora Fuckel (1)

Trachyspora alchemillae (Pers.) Fuckel

9. Pileolariaceae (Arthur) Cummins \& Y. Hirats., llustr. Gen. Rust Fungi, rev. Edn (St. Paul): 14. 1983. emend. Aime \& McTaggart

Pileolaria Castagne (2)

Pileolaria indica Syd.

Pileolaria pistaciae F.L. Tai \& C.T. Wei

10. Pucciniastraceae Gäum. ex Leppik, Ann. bot. fenn. 9: 139. 1972. emend. Aime \& McTaggart Hyalopsora Magnus (2)

Hyalopsora orientalis Chona \& Munjal

Hyalopsora polypodii (Pers.) Magnus 
Melampsoridium Kleb. (4)

Melampsoridium betulinum (Pers.) Kleb.

Melampsoridium hiratsukanum S. Ito ex Hirats. f.

Melampsoridium indicum Sathe

Melampsoridium inerme Suj. Singh \& P.C. Pandey

Pucciniastrum G.H. Otth (6)

Pucciniastrum aceris Syd.

Pucciniastrum agrimoniae (Dietel) Tranzschel

Pucciniastrum celastri Syd. \& P. Syd.

Pucciniastrum coriariae Dietel

Pucciniastrum coryli Kom.

Pucciniastrum gaultheriae Syd. \& P. Syd.

Peridermium (Link) J.C. Schmidt \& Kunze (7)

Peridermium brevius Barclay

Peridermium cedri Barclay

Peridermium ephedrae Cooke

Peridermium himalayense Bagchee

Peridermium orientale Cooke

Peridermium piceae Barclay

Peridermium thomsonii Berk.

11. Pucciniaceae Chevall., Fl. gén. env. Paris (Paris) 1: 413. 1826. emend. Aime \& McTaggart

Caeoma Link (4)

Caeoma himalayense Suj. Singh, S.N. Khan \& B.M. Misra

Caeoma scopariae K.N. Rao

Caeoma euphorbiae-geniculatae Ramachar \& Bhagyan.

Caeoma indicum Rajendren

Chrysocelis Lagerh. \& Dietel (1)

Chrysocelis butleri (Dietel, Syd. \& P. Syd.) G.F. Laundon

Corbulopsora Cummins (1)

Corbulopsora cumminsii Thirum.

Endophyllum Lév. (8)

Endophyllum cassiae Nag Raj, Govindu \& Thirum.

Endophyllum cassiae (Bres.) F. Stevens \& Mendiola

Endophyllum elaeagni-latifoliae (Petch) Gokhale, Thirum. \& Patel

Endophyllum emiliae-sonchifoliae Nag Raj, Govindu \& Thirum.

Endophyllum heliotropii Thirum. \& Naras.

Endophyllum kaernbachii (Henn.) F. Stevens \& Mendiola

Endophyllum maheshwarii Hard. Singh \& Jalan

Endophyllum macowanianum (Thüm.) Pole-Evans

Endophyllum spilanthis Thirum. \& Govindu

Gambleola Massee (1)

Gambleola cornuta Massee

Hapalophragmium Syd. \& P. Syd. (4)

Hapalophragmium anamalaiense T.S. Ramakr. \& K. Ramakr.

Hapalophragmium mysorense Thirum.

Hapalophragmium ponderosum Syd., P. Syd. \& E.J. Butler

Hapalophragmium tandonii Mitter

Kernella Thirum. (1)

Kernella lauricola (Thirum.) Thirum.

Puccinia Pers. (279)

Puccinia acanthospermi Henn. 
Puccinia acrophila Peck

Puccinia actaeae-agropyri E. Fisch.

Puccinia adjuncta Mitter

Puccinia aggregata Syd. \& P. Syd.

Puccinia agrostidis Plowr.

Puccinia ahmadiana Syd.

Puccinia ainsliaeae P. Syd. \& Syd.

Puccinia altii Rud.

Puccinia amphilophidis Doidge

Puccinia angelicae (Schumach.) Fuckel

Puccinia anodae P. Syd. \& Syd.

Puccinia antirrhini Dietel \& Holw.

Puccinia apii Desm.

Puccinia apludae Syd. \& P. Syd.

Puccinia arachidis Speg.

Puccinia arenariae (Schumach.) J. Schröt.

Puccinia argentata (Schultz) G. Winter

Puccinia aristidae Tracy

Puccinia aristidicola Henn.

Puccinia arthraxonis-ciliaris Cummins

Puccinia arundinellae Barclay

Puccinia asterum (Schwein.) F. Kern

Puccinia atropuncta Peck \& Clinton

Puccinia azanzae Yadav

Puccinia baradensis P.B. Chavan \& U.V. Kulk.

Puccinia barbeyi (Roum.) Magnus

Puccinia behenis G.H. Otth

Puccinia belamcandae Dietel

Puccinia bellurensis Thirum.

Puccinia betae-bengalensis Mundk. \& Thirum.

Puccinia bistortae (F. Strauss) DC.

Puccinia blepharidis Henn.

Puccinia bottomleyae Doidge

Puccinia brachypodii G.H. Otth

Puccinia bulbostylidis Doidge

Puccinia bupleuri F. Rudolphi

Puccinia butleri Syd. \& P. Syd.

Puccinia cacao McAlpine

Puccinia caheunsis Ell. \& Ev.

Puccinia calcitrapae var. filicinae Barclay

Puccinia calcitrapae DC.

Puccinia calosperma Syd., P. Syd. \& E.J. Butler

Puccinia calthae Link

Puccinia caricis-filicinae Barclay

Puccinia caricis-nubigenae Padwick \& A. Khan

Puccinia cenchri Dietel \& Holw.

Puccinia cephalandrae-indicae Syd. \& P. Syd.

Puccinia chaerophylli Purton

Puccinia chloridis-incompletae T.S. Ramakr., Sriniv. \& Sundaram

Puccinia chrysanthemi Roze

Puccinia circaeae Pers.

Puccinia citrina P. Syd. \& Syd. 
Puccinia citrulli Syd., P. Syd. \& E.J. Butler

Puccinia citrullina Raghun. \& K. Ramakr. ex Hosag. \& Raghun.

Puccinia collettiana Barclay

Puccinia conclusa Thüm.

Puccinia congesta Berk. \& Broome

Puccinia coronata Corda

Puccinia courtoisiae (Syd. \& P. Syd.) Syd.

Puccinia crepidis-japonicae (Lindr.) Dietel

Puccinia crepidis-sibiricae Lindr.

Puccinia cressae Lagerh.

Puccinia ctenolepidis Ramachar \& Bagyan.

Puccinia curculiginis Racib.

Puccinia curcumae T.S. Ramakr. \& Sundaram

Puccinia cynodontis Lacroix ex Desm.

Puccinia cyperi Arthur

Puccinia cyperi-tagetiformis (Henn.) F. Kern

Puccinia dactylidina Bubák

Puccinia deodikarii K.R.G. Nair

Puccinia desertorum Syd. \& P. Syd.

Puccinia digitariae Ramachar \& George

Puccinia digitariae-biformis P.B. Chavan \& Hosag.

Puccinia digitariae-vestitae Ramachar \& Cummins

Puccinia dioicae Magnus

Puccinia dioscoreae Kom.

Puccinia dovrensis A. Blytt

Puccinia drabae F. Rudolphi

Puccinia droogensis E.J. Butler

Puccinia abutilonis Berk. \& Broome

Puccinia aristidae var. chaetariae Cummins \& S.M. Husain

Puccinia bulbocastani (A. Cumino) Fuckel

Puccinia canaliculata (Schwein.) Lagerh.

Puccinia centaureae $\mathrm{H}$. Mart.

Puccinia chrysopogoni Barclay

Puccinia coronata f.sp. avenae P. Syd. \& Syd.

Puccinia dissiliens Cooke

Puccinia duthiei Ellis \& Tracy

Puccinia ellisii De Toni

Puccinia elytrariae Henn.

Puccinia engleriana Henn.

Puccinia enteropogonis P. Syd. \& Syd.

Puccinia eragrostidis Petch

Puccinia eremuri Kom.

Puccinia erianthi Padwick \& A. Khan

Puccinia eulaliae Barclay

Puccinia eutela Syd.

Puccinia exhauriens Thüm.

Puccinia expallens Syd. \& P. Syd.

Puccinia echinopis DC.

Puccinia excelsa Barclay

Puccinia fagopyri Barclay

Puccinia fagopyricola Jørst.

Puccinia ferruginosa P. Syd. \& Syd. 
Puccinia festucae Plowr.

Puccinia fimbristylidis Arthur

Puccinia fimbristylidis-ferrugineae Ramachar, Bhagyan. \& A. Kumar

Puccinia flaccida Berk. \& Broome

Puccinia flavipes Syd. \& P. Syd.

Puccinia fuirenicola Arthur

Puccinia fusca G. Winter

Puccinia garnotiae T.S. Ramakr. \& Sundaram

Puccinia gentianae (F. Strauss) Link

Puccinia geranii-silvatici $\mathrm{P}$. Karst.

Puccinia gerberae Pole-Evans

Puccinia gouaniae Holw.

Puccinia gracilenta Syd., P. Syd. \& E.J. Butler

Puccinia graminis f. avenae Erikss. \& Henning

Puccinia graminis Pers.

Puccinia graminis f. agropyri P.R. Mehta \& R. Prasad

Puccinia graminis f. poae Erikss. \& Henning

Puccinia graminis f. tritici Erikss. \& Henning

Puccinia gymnopetali-wightii T.S. Ramakr., Sriniv. \& Sundaram

Puccinia helianthi Schwein.

Puccinia heraclei Grev.

Puccinia heracleicola Cummins

Puccinia herqueri

Puccinia heterospora Berk. \& M.A. Curtis

Puccinia heucherae (Schwein.) Dietel

Puccinia hieracii (Röhl.) H. Mart.

Puccinia himachalensis A.K. Gautam \& S. Avasthi

Puccinia holboelliae-latifoliae Cummins

Puccinia hookeri P. Syd. \& Syd.

Puccinia hordei G.H. Otth

Puccinia hyderabadensis Bagyan. \& Ravinder

Puccinia hydrocotyles (Mont.) Cooke

Puccinia hypoxidis McAlpine

Puccinia inayatii Syd. \& P. Syd.

Puccinia insidiosa Berk.

Puccinia intermixta Peck

Puccinia invenusta Syd. \& P. Syd.

Puccinia investita Schwein.

Puccinia iridis Wallr.

Puccinia isachnes Petch

Puccinia jagopyri Barclay

Puccinia jasminicola T.S. Ramakr. \& K. Ramakr.

Puccinia joerstadii S. Ahmad

Puccinia kalchbrenneri De Toni

Puccinia kenmorensis Cummins

Puccinia kraussiana Cooke

Puccinia kuehnii (W. Krüger) E.J. Butler

Puccinia kunthiana T.S. Ramakr., Sriniv. \& Sundaram

Puccinia lantanae Farl.

Puccinia lateripes Berk. \& Ravenel

Puccinia lateritia Berk. \& M.A. Curtis

Puccinia launaeae Maire 
Puccinia leiocarpa Thirum.

Puccinia leonotidicola Henn.

Puccinia leucadis P. Syd. \& Syd.

Puccinia leucophaea Syd., P. Syd. \& E.J. Butler

Puccinia leveillei Mont.

Puccinia levis (Sacc. \& Bizz.) Magnus

Puccinia libani Magnus

Puccinia liberta F. Kern

Puccinia ligustici Ellis \& Everh.

Puccinia linkii Klotzsch

Puccinia lithospermi Ellis \& Kellerm.

Puccinia longirostris Kom

Puccinia luculenta (Syd. \& P. Syd.) T.S. Ramakr. \& K. Ramakr.

Puccinia macrorhynchi Rabenh.

Puccinia malvacearum Bertero ex Mont.

Puccinia melanocephala Syd. \& P. Syd.

Puccinia melasmioides Tranzschel

Puccinia menthae Pers.

Puccinia merrillii Henn.

Puccinia microspora Dietel

Puccinia minutissima Arthur

Puccinia monticola Kom.

Puccinia mysorensis Syd.

Puccinia melothriicola Syd. \& P. Syd.

Puccinia nakanishikii Dietel

Puccinia nepalensis Barclay \& Dietel

Puccinia neyraudiae Syd. \& P. Syd.

Puccinia nitida Barclay

Puccinia oahuensis Ellis \& Everh.

Puccinia obscura J. Schröt.

Puccinia ocimi Doidge

Puccinia oligocarpa Syd., P. Syd. \& E.J. Butler

Puccinia operculinae T.S. Ramakr. \& Sundaram

Puccinia operta Mundk. \& Thirum.

Puccinia opizii Bubák

Puccinia oplismeni Syd. \& P. Syd.

Puccinia oreogeta Syd.

Puccinia oryzopsidis Syd., P. Syd. \& E.J. Butler

Puccinia ottochloae T.S. Ramakr.

Puccinia oxalidis Dietel \& Ellis

Puccinia pachypes Syd. \& P. Syd.

Puccinia pacifica Blasdale ex Arthur

Puccinia padwickii Cummins

Puccinia panici-montani Fujik. ex Ramachar \& Cummins

Puccinia paspali Tracy \& Earle

Puccinia pectiniformis T.S. Ramakr., Sriniv. \& Sundaram

Puccinia peradeniyae Demers \& Castl.

Puccinia peraffinis Syd. \& P. Syd.

Puccinia phragmitis (Schumach.) Tul.

Puccinia phyllocladiae Cooke

Puccinia phyllostachydis Kusano

Puccinia pieridiz Hazel. 
Puccinia pimpinellae (F. Strauss) Link

Puccinia plicata Kom.

Puccinia pogonatheri Petch

Puccinia polliniae Barclay

Puccinia polliniae-quadrinervis Dietel

Puccinia polygoni-amphibii Pers.

Puccinia polygoni-weyrichii Miyabe

Puccinia polysora Underw.

Puccinia porri (Sowerby) G. Winter

Puccinia praecox Bubák

Puccinia prainiana Barclay

Puccinia prenanthis-purpureae (DC.) Lindr.

Puccinia princeps Syd. \& P. Syd.

Puccinia propinqua Syd., P. Syd. \& E.J. Butler

Puccinia prostii Moug.

Puccinia pseudocesatii Cummins

Puccinia pulverulenta Grev.

Puccinia pulvinata Rabenh.

Puccinia punctata Link

Puccinia purpurea Cooke

Puccinia pusilla Syd. \& P. Syd.

Puccinia recondita Roberge ex Desm.

Puccinia ribis DC.

Puccinia recondita var. simlensis A.P. Misra, S.T. Ahmad \& Sheodh. Singh

Puccinia ribis-caricis Kleb.

Puccinia rhynchosporae Syd. \& P. Syd.

Puccinia romagnoliana Maire \& Sacc.

Puccinia roscoeae Barclay

Puccinia rostrata Cooke

Puccinia rottboelliae P. Syd. \& Syd.

Puccinia ruelliae Lagerh.

Puccinia rufipes Dietel

Puccinia sacchari Patel, Kamat \& Y.A. Padhye

Puccinia sonchi Roberge ex Desm.

Puccinia satarensis P.B. Chavan \& Bakare

Puccinia saussureae Thüm.

Puccinia saviculae Grev.

Puccinia saxifragae-ciliatae Barclay

Puccinia schedonnardi Kellerm. \& Swingle

Puccinia schirajewskii Tranzschel

Puccinia scirpi DC.

Puccinia senecionis-scandentis Lindr.

Puccinia shiraiana P. Syd.

Puccinia silvaticella Arthur \& Cummins

Puccinia solanacearum Sacc. \& P. Syd.

Puccinia solani-giganteae T.S. Ramakr. \& K. Ramakr.

Puccinia solmsii (Kuntze) Sacc. \& P. Syd.

Puccinia sorghi Schwein.

Puccinia spongiosa Berk. \& Broome

Puccinia stenotaphricola J. Walker

Puccinia striiformis Westend.

Puccinia striiformis f. muehlenbergii 
Puccinia suaveolens (Pers.) Rostr.

Puccinia substriata Ellis \& Barthol.

Puccinia swertiae G. Winter

Puccinia tanaceti DC.

Puccinia terminaliae T.S. Ramakr. \& Sundaram

Puccinia thlaspeos Ficinus \& C. Schub.

Puccinia thomasiana T.S. Ramakr. \& K. Ramakr.

Puccinia thunbergiae Cooke

Puccinia thwaitesii Berk.

Puccinia tiliaefolia T.S. Ramakr. \& Sundaram

Puccinia tragiae Cooke

Puccinia tricholepidis Syd.

Puccinia trollii P. Karst.

Puccinia turgida P. Syd. \& Syd.

Puccinia tweediana T.S. Ramakr. \& K. Ramakr.

Puccinia unica var. chica Cummins \& S.M. Husain

Puccinia urticae Barclay

Puccinia ustalis Berk.

Puccinia verruca Thüm.

Puccinia vernoniae-monosis T.S. Ramakr. \& K. Ramakr.

Puccinia versicolor Dietel \& Holw.

Puccinia violae (Schumach.) DC.

Puccinia volutarellae Thirum.

Puccinia wangikarii Somani

Puccinia wattiana Barclay

Puccinia weyrehii Miyabe

Puccinia woodii (Kalchbr. \& Cooke) P. Syd. \& Syd.

Puccinia xanthii Schwein.

Puccinia xanthocarpi R.Y. Roy \& P.C. Gupta

Puccinia xanthopoda Syd. \& P. Syd.

Puccinia xanthosperma Syd. \& P. Syd.

Puccinia zingiberis T.S. Ramakr.

Pucciniosira Lagerh (1)

Pucciniosira tuberculata (Ellis \& Kellerm.) Buriticá \& J.F. Hennen

Ramakrishnania Ramachar \& Bhagyan. (1)

Ramakrishnania ixorae Ramachar \& Bhagyan.

Trochodium Syd. \& P. Syd. (2)

Trochodium ajrekarii Gharse

Trochodium sampathense Thirum.

Uromyces (Link) Unger (89)

Uromyces achrous Syd. \& P. Syd.

Uromyces aconiti Fuckel

Uromyces acori T.S. Ramakr. \& Rangaswami

Uromyces agropyri Barclay

Uromyces aloes (Cooke) Magnus

Uromyces ambiens Cooke

Uromyces amphilophis-insculptae T.S. Ramakr., Sriniv. \& Sundaram

Uromyces andropogonis Tracy

Uromyces andropogonis-annulati Syd., P. Syd. \& E.J. Butler

Uromyces anotidis-monospermatis T.S. Ramakr. \& Sundaram

Uromyces anthyllidis (Grev.) J. Schröt.

Uromyces apludae Syd., P. Syd. \& E.J. Butler 
Uromyces appendiculatus (Pers.) Link

Uromyces behenis (DC.) Unger

Uromyces bidentis Lagerh.

Uromyces blainvilleae Berk.

Uromyces callicarpae (Petch) Fujik. ex S. Ito

Uromyces capitatus Syd. \& P. Syd.

Uromyces ciceris-arietini (Grognot) Jacz. \& G. Boyer

Uromyces clignyi Pat. \& Har.

Uromyces clivalis Mitter

Uromyces commelinae Cooke

Uromyces coronatus Yoshin.

Uromyces dactylidis G.H. Otth

Uromyces decoratus Syd. \& P. Syd.

Uromyces dolicholi Arthur

Uromyces eragrostidis Tracy

Uromyces eriochloae (Syd. \& P. Syd.) Syd., P. Syd. \& E.J. Butler

Uromyces euphorbiae Cooke \& Peck

Uromyces fritillariae Thüm.

Uromyces geranii (DC.) G.H. Otth \& Wartm.

Uromyces haussknechtii Tranzschel

Uromyces hedysari-obscuri (DC.) Carestia \& Picc.

Uromyces heterogeneus Cooke

Uromyces hobsonii Vize

Uromyces hyderabadensis Ramachar, K.N. Rao \& Bagyan.

Uromyces indigoferae f.sp. tinctoriae L.M. Joshi \& A.R. Reddy

Uromyces ignobilis (Syd. \& P. Syd.) Arthur

Uromyces inayatii Syd. \& P. Syd.

Uromyces indicus Pat.

Uromyces indigoferae Dietel \& Holw.

Uromyces lapponicus Lagerh.

Uromyces lespedezae-procumbentis (Schwein.) Lagerh.

Uromyces lespedezae-sericeae S. Ahmad

Uromyces lineolatus (Desm.) J. Schröt.

Uromyces loculiformis T.S. Ramakr. \& K. Ramakr.

Uromyces macintirianus Barclay

Uromyces minor J. Schröt.

Uromyces mucunae Rabenh.

Uromyces muscari (Duby) Niessl

Uromyces mussooriensis Syd. \& P. Syd.

Uromyces nilagiricus T.S. Ramakr. \& K. Ramakr.

Uromyces orientalis Syd. \& P. Syd.

Uromyces orthosiphonis T.S. Ramakr. \& Sriniv.

Uromyces ottochloae Ramakr. T.S.

Uromyces panici-sanguinalis Rangel

Uromyces pavgii R.N. Goswami \& Ngachan

Uromyces peglerae Pole-Evans

Uromyces pianhyensis Henn.

Uromyces pisi-sativi (Pers.) Liro

Uromyces polygoni-avicularis (Pers.) G.H. Otth

Uromyces pontederiicola Speg.

Uromyces poonensis W.D. More \& Moniz

Uromyces proeminens (DC.) Lév. 
Uromyces pseudarthriae Cooke

Uromyces ramacharii Ravinder \& Bagyan.

Uromyces rottboelliae Arthur

Uromyces rugulosus Pat.

Uromyces rumicis (Schumach.) G. Winter

Uromyces satarensis P.B. Chavan \& Bakare

Uromyces schoenanthi Syd. \& P. Syd.

Uromyces setariae-italicae Yoshino

Uromyces sommerfeltii Hyl., Jørst. \& Nannf.

Uromyces spegazzinii (De Toni) Arthur

Uromyces sphaeropleus Cooke

Uromyces sporgoni Clint \& Peck. subsp. asiaticus

Uromyces striatus J. Schröt.

Uromyces strobilanthis Barclay

Uromyces superfluus P. Syd. \& Syd.

Uromyces tenuicutis McAlpine

Uromyces triandrae T.S. Ramakr. \& Sriniv.

Uromyces trichoneurae Doidge

Uromyces trifolii (R. Hedw.) Lév.

Uromyces trigonellae Pass.

Uromyces tripogonicola Payak \& Thirum.

Uromyces trollii-caroli Ulbr.

Uromyces valerianae (Schumach.) Fuckel

Uromyces valerianae-wallichii (Dietel) Arthur \& Cummins

Uromyces vestergrenii $\mathrm{P}$. Syd. \& Syd.

Uromyces viciae-fabae (Pers.) J. Schröt.

Uromyces vignae Barclay

Uromyces vossiae Barclay

Uromyces wedeliae-biflorae Boedijn

Uromyces wellingtonicus T.S. Ramakr. \& K. Ramakr.

Xenostele Syd. \& P. Syd. (1)

Xenostele litseae (Pat.) Syd. \& P. Syd.

12. Raveneliaceae Leppik, Ann. bot. fenn. 9: 139. 1972. emend. Aime \& McTaggart

Chaconia Juel (1)

Chaconia butleri (Syd. \& P. Syd.) Mains

Didymopsorella Thirum. (1)

Didymopsorella macrospora (Mundk. \& Thirum.) Thirum.

Diorchidium Kalchbr. (3)

Diorchidium levigatum Syd., P. Syd. \& E.J. Butler

Diorchidium orientale Syd., P. Syd. \& E.J. Butler

Diorchidium tricholaenae Syd. \& P. Syd.

Gymnopuccinia K. Ramakr. (1)

Gymnopuccinia pulneyensis K. Ramakr.

Kernkampella Rajendren (6)

Kernkampella breyniae (Syd. \& P. Syd.) Rajendren

Kernkampella breyniae-patentis (Mundk. \& Thirum.) Rajendren

Kernkampella coimbatorica (T.S. Ramakr. \& Sundaram) G.F. Laundon

Kernkampella emblicae (Syd. \& P. Syd.) G.F. Laundon

Kernkampella kirganeliae (Mundk. \& Thirum.) G.F. Laundon

Kernkampella phyllanthi (Mundk. \& Thirum.) G.F. Laundon 
Maravalia Arthur (11)

Maravalia achroa (Syd.) Arthur \& Cummins

Maravalia aulica (Syd.) Y. Ono

Maravalia ascotela (Syd.) Mains

Maravalia echinulata (Niessl ex Rabenh.) Y. Ono

Maravalia fici (Mundk. \& Thirum.) Y. Ono

Maravalia gentilis (Syd.) Y. Ono

Maravalia ichnocarpi (Barclay) Sathe

Maravalia millettiae Yadav \& Thirum.

Maravalia milletticola Y. Ono \& J.F. Hennen

Maravalia mimusops (Cooke) Y. Ono

Maravalia pterocarpi (Thirum.) Thirum.

Scopella Mains (1)

Scopella dalbergiae (T.S. Ramakr. \& K. Ramakr.) Ragunathan \& K. Ramakr.

Olivea Arthur (3)

Olivea colebrookiana Thirum. \& Yadav

Olivea isonandrae Hosag.

Olivea tectonae (Racib.) Thirum.

Ravenelia Berk. (33)

Ravenelia acaciae-arabicae Mundk. \& Thirum.

Ravenelia acaciae-caesiae Tyagi \& S.S. Prasad

Ravenelia acaciae-concinnae Mundk. \& Thirum.

Ravenelia acaciae-intsiae B.V. Patil \& Thirum.

Ravenelia acaciae-pennatulae Dietel

Ravenelia acaciae-pennatulae Dietel

Ravenelia acaciae-sumae Thirum. \& Mundk.

Ravenelia acaciae-senegalae Sanwal

Ravenelia acaciicola Sanwal

Ravenelia aculeifera Berk.

Ravenelia ajmerensis Sanwal

Ravenelia albiziae-amarae Bacc.

Ravenelia burmanica Thaung

Ravenelia cassiicola G.F. Atk.

Ravenelia clemensae Syd.

Ravenelia deformis Tyagi \& S.S. Prasad

Ravenelia tephrosiicola (Henn.) Hirats. f.

Ravenelia esculenta Naras. \& Thirum.

Ravenelia evansii Syd. \& P. Syd.

Ravenelia fragrans Long

Ravenelia hansfordii Cummins

Ravenelia hobsonii Cooke

Ravenelia indica Berk.

Ravenelia japonica Dietel \& P. Syd.

Ravenelia karadensis P.B. Chavan \& U.V. Kulk.

Ravenelia mitis Syd. \& P. Syd.

Ravenelia mitteri Syd.

Ravenelia odoratissimae Tyagi \& S.S. Prasad

Ravenelia ornata Syd. \& P. Syd.

Ravenelia parasnathii Yadav

Ravenelia radhanagarensis Patil

Ravenelia satarensis P.B. Chavan \& U.V. Kulk.

Ravenelia sayeedii M.A. Salam \& Ramachar 
Ravenelia sessilis Berk.

Ravenelia spicigerae B.V. Patil \& Thirum.

Ravenelia stictica Berk. \& Broome

Ravenelia sumatii S.D. Patil \& Date

Ravenelia tandonii Syd.

Ravenelia taslimii Mundk.

Ravenelia versatilis (Peck) Dietel

Prospodium Arthur (2)

Prospodium erebia (Syd. \& P. Syd.) Bagyan. \& Ravinder

Prospodium tirumalense Bagyan., Ravinder \& P. Ramesh

13. Skierkaceae (Arthur) Aime \& McTaggart, Fungal Systematics and Evolution 7: 21-47, 2020.

Skierka Racib. (3)

Skierka agallochae Racib.

Skierka himalayensis A.K. Gautam \& S. Avasthi

Skierka toddaliae (Petch) Hirats.

14. Sphaerophragmiaceae Cummins \& Y. Hirats., Illustr. Gen. Rust Fungi, rev. Edn (St. Paul): 15. 1983. emend. Aime \& McTaggart

Sphaerophragmium Magnus (1)

Sphaerophragmium acaciae (Cooke) Magnus

15. Tranzscheliaceae (Arthur) Aime \& McTaggart, Fungal Systematics and Evolution 7: 21-47, 2020

Leucotelium Tranzschel (1)

Leucotelium pruni-persicae (Hori) Tranzschel

Tranzschelia Arthur (2)

Tranzschelia discolor (Fuckel) Tranzschel \& M.A. Litv.

Tranzschelia pruni-spinosae (Pers.) Dietel

16. Zaghouaniaceae P. Syd. \& Syd., Monogr. Uredin. (Lipsiae) 3(3): 586. 1915. emend. Aime \& McTaggart

Cystopsora E.J. Butler (1)

Cystopsora antidesmatis T.S. Ramakr. \& Sundaram

Elateraecium Thirum., F. Kern \& B.V. Patil (1)

Elateraecium salaciicola Thirum., F. Kern \& B.V. Patil

Hemileia Berk. \& Broome (10)

Hemileia canthii Berk. \& Broome

Hemileia gardeniae-thunbergiae (Henn.) Maubl. \& Roger

Hemileia holarrhenae Syd. \& P. Syd.

Hemileia indica Massee

Hemileia jasmini C.S. Krishnam. \& Rangaswami

Hemileia mysorensis Thirum. \& Gopalakrishn.

Hemileia pavetticola Maubl. \& Roger

Hemileia thomasii Thirum. \& Naras.

Hemileia vastatrix Berk. \& Broome

Hemileia wrightiae (Racib.) Racib.

Zaghouania Pat. (1)

Zaghouania oleae (E.J. Butler) Cummins

\section{Pucciniales genera incertae sedis}

Aecidium Vuill. 
Aecidium adhatodae Syd. \& P. Syd.

Aecidium aechmantherae Syd. \& P. Syd.

Aecidium ajugae Syd. \& P. Syd.

Aecidium anaphalidis-leptophyllae T.S. Ramakr., Sriniv. \& Sundaram

Aecidium argyreiae Berk. \& Broome

Aecidium brasiliense Dietel

Aecidium callianthum Syd.

Aecidium campanulae Pandotra \& K.S.M. Sastry

Aecidium carviae Sathe

Aecidium cassiae-torae P.B. Chavan \& Bakare

Aecidium cinnamomi Racib.

Aecidium cleomes Ellis \& H.W. Anderson

Aecidium clerodendri Henn.

Aecidium colchici-aurei Ulbr.

Aecidium crassocephali Wakef. \& Hansf.

Aecidium crini Kalchbr.

Aecidium crypticum Kalchbr. \& Cooke

Aecidium cuspidatum T.S. Ramakr., Sriniv. \& Sundaram

Aecidium delphinii Barthol.

Aecidium deutziae Dietel

Aecidium dichrocephalae Henn.

Aecidium diospyri A.L. Sm.

Aecidium distinctum Arthur \& Cummins

Aecidium elaeocarpi-tuberculati Hosag.

Aecidium esculentum Barclay

Aecidium flavescens Barclay

Aecidium garciniae Sundaram \& A.V. Rao

Aecidium girardiniae Syd. \& P. Syd.

Aecidium gymnematis T.S. Ramakr. \& Sundaram

Aecidium hartwegiae Thüm.

Aecidium hedyotidis Syd.

Aecidium hemidesmi Syd. \& P. Syd.

Aecidium hemigraphidis B.V. Patil \& Thirum.

Aecidium infrequens Barclay

Aecidium innatum Syd., P. Syd. \& E.J. Butler

Aecidium inquitosense P. Henn.

Aecidium kamatii Sathe

Aecidium latifolium Massee

Aecidium leeae M.A. Salam \& Ramachar

Aecidium lepidagathis Syd. \& P. Syd.

Aecidium leucadinum Mitter

Aecidium lophanthi Henn.

Aecidium lophopetali Wakef.

Aecidium marsdeniae T.S. Ramakr. \& K. Ramakr.

Aecidium melaleucum Syd. \& P. Syd.

Aecidium meliosmae-wightii T.S. Ramakr., Sriniv. \& Sundaram

Aecidium memecyli Thirum.

Aecidium microrhynchi Henn.

Aecidium miliare Berk. \& Broome

Aecidium montanum E.J. Butler

Aecidium mori Barclay

Aecidium morobeanum Cummins 
Aecidium myriactidis (Barclay) P. Syd. \& Syd.

Aecidium nummulare Berk.

Aecidium ocimi Henn.

Aecidium orbiculare Barclay

Aecidium osmanthi Syd., P. Syd. \& E.J. Butler

Aecidium painavuense Hosag.

Aecidium paramignyae Racib.

Aecidium pavoniae-odoratae T.S. Ramakr., Sriniv. \& Sundaram

Aecidium peristrophes Syd. \& P. Syd.

Aecidium petchii Sacc. \& Trotter

Aecidium plectranthicola Cummins

Aecidium plectroniae Cooke

Aecidium ponderosum Syd. \& P. Syd.

Aecidium poonense Sathe

Aecidium pulneyense T.S. Ramakr. \& Sriniv.

Aecidium pupaliae Prasad, L.C. Sharma \& R.D. Singh

Aecidium pygei Syd. \& P. Syd.

Aecidium quintum Syd. \& P. Syd.

Aecidium randiae Henn.

Aecidium rhododendri Barclay

Aecidium rhynchosiae Bagyan. \& Ramachar

Aecidium rhytismoideum Berk. \& Broome

Aecidium salamii G.F. Laundon

Aecidium satarense P.B. Chavan \& S.K. Patil

Aecidium saussureae Johanson

Aecidium scutellariae Syd. \& P. Syd.

Aecidium sinhagadense Sathe

Aecidium solani Mont.

Aecidium spilanthis T.S. Ramakr. \& Sundaram

Aecidium stewartianum Cummins

Aecidium stewartii Arthur \& Cummin

Aecidium stranvaesiae Syd. \& P. Syd.

Aecidium strobilanthis Barclay

Aecidium tandonii Mitter

Aecidium terminaliae T.S. Ramakr. \& K. Ramakr.

Aecidium travancoricum T.S. Ramakr.

Aecidium tricholepidis P.B. Chavan \& Bakare

Aecidium tubulosum Pat. \& Gaillard

Aecidium urceolatum Cooke

Aecidium urgineae Henn. \& Pole-Evans

Aecidium vangueriae Cooke

Aecidium verbenae Speg.

Aecidium vernoniae-cinereae Petch

Aecidium walayarense T.S. Ramakr. \& Sundaram

Aecidium withaniae Thüm.

Nyssopsora Arthur

Nyssopsora cedrelae (Hori) Tranzschel

Nyssopsora thirumalacharii R.N. Goswami \& Ngachan

Nyssopsora thwaitesii (Berk. \& Broome) Syd.

Phragmotelium Syd.

Phragmotelium burmanicum (Syd. \& P. Syd.) Syd.

Phragmidium mysorense (Thirum. \& Mundk.) B. Ali \& Berndt 
Tunicopsora Suj. Singh \& P.C. Pandey

Tunicopsora bagchii Suj. Singh \& P.C. Pandey

\section{Uraecium Arthur}

Uraecium nothopegiae T.S. Ramakr. \& K. Ramakr.

Uredo Pers.

Uredo acaciae-concinnae Kapoor bis \& D.K. Agarwal

Uredo acalyphae-fruticosae T.S. Ramakr., Sriniv. \& Sundaram

Uredo allmaniae P.B. Chavan \& U.V. Kulk.

Uredo alpestris J. Schröt.

Uredo amomi Petch

Uredo apludae Barclay

Uredo arachidis Lagerh.

Uredo brachylepidis T.S. Ramakr. \& Sundaram

Uredo cajani Syd. \& P. Syd.

Uredo carissae Thirum.

Uredo carissae-occidentalis Chavan \& Kulkurni

Uredo cassiae K.N. Rao

Uredo cassiae-occidentalis T.S. Ramakr.

Uredo celastri Arthur \& Cummins

Uredo celastri-paniculatae T.S. Ramakr. \& Sundaram

Uredo chasaliae Petch

Uredo citri Vaheed.

Uredo davaoensis Syd. \& P. Syd.

Uredo dalbergiae-latifoliae Hosag. \& N.C. Nair

Uredo deutziae Barclay

Uredo dioscoreae Henn.

Uredo dioscoreae-sativae Syd. \& P. Syd.

Uredo echinulata (Niessl.) Syd.

Uredo ehretiae Barclay

Uredo elephantopodis Petch

Uredo elettariae Thirum.

Uredo emiliae-scabrae T.S. Ramakr. \& Sundaram

Uredo exasperata (Cooke) Sacc.

Uredo garugae Sundaram

Uredo gayanae J.C. Lindq.

Uredo gharsei Sathe \& Rahalkar

Uredo gomphrenae Barclay

Uredo hygrophilicola G.F. Laundon \& Ponnappa

Uredo hyperici-mysorensis Petch

Uredo khandalensis Sathe \& Rahalkar

Uredo launeae-coromandelicae Chavan \& Bakare

Uredo lipocarphae Syd. \& P. Syd.

Uredo malabarica T.S. Ramakr. \& K. Ramakr.

Uredo mannanurensis K.N. Rao

Uredo microspora (Vize) Sacc.

Uredo mundkurii P.B. Chavan

Uredo myriactidis Sundaram

Uredo neilgherriensis T.S. Ramakr.

Uredo niterogensis Rangel

Uredo ochnae K.N. Rao

Uredo ophiorrhizae Petch

Uredo ophiuri Syd., P. Syd. \& E.J. Butler 
Uredo paederiae Syd. \& P. Syd.

Uredo pallidula Cooke \& Massee

Uredo panacis Syd. \& P. Syd.

Uredo paspali Pandotra

Uredo paspali-scrobiculati Syd. \& P. Syd.

Uredo phyllanthi-niruris M.S. Patil

Uredo pileae Barclay

Uredo pouzolziae Syd. \& P. Syd.

Uredo pterocarpi T.S. Ramakr.

Uredo punctoidea Cooke

Uredo ravennae Maire

Uredo rhinacanthi T.S. Ramakr. \& Sundaram

Uredo rottboelliae Dietel

Uredo shuteriae T.S. Ramakr.

Uredo sesbaniae Henn.

Uredo setariae-tomentosae Ramachar, Bhagyan. \& A. Kumar

Uredo sissoo Syd., P. Syd. \& E.J. Butler

Uredo spinulosa (Cooke) Sacc.

Uredo tephrosiae Rabenh.

Uredo terminaliae Henn.

Uredo terminaliae-paniculatae T.S. Ramakr. \& K. Ramakr.

Uredo thelypteridis Yadav

Uredo thelypteridis var. thelypteridis Yadav

Uredo verecunda Syd.

Uredo victoriae Cummins

\section{Phylogenetic analysis}

The phylogenetic analyses based on the combined LSU and ITS rDNA sequence dataset comprised 189 taxa including various genera from 13 families belonging to Pucciniales were assessed with Taphrina pruni CBS 358.35 as an outgroup taxon. RAxML analysis of the combined dataset produced the best tree with a final ML optimization likelihood value of -34581.032655 . The genera from different families included in the phylogenetic analyses are Coleosporiaceae, Cronartiaceae, Crossopsoraceae, Melampsoraceae, Milesinaceae, Ochropsoraceae, Phakopsoraceae, Phragmidiaceae, Pileolariaceae, Pucciniastraceae, Pucciniaceae, Raveneliaceae and Tranzscheliaceae. The genera Coleosporium, Chrysomyxa clustered together in Coleosporiaceae clade with significant support from ML 93\% and Cronartia formed a distinct clade sharing a sister group relationship with Coleosporium and Chrysomyxa. The Melampsoraceae clade is supported by taxa from Melampsora with significant support from ML 100\%. The Pucciniastraceae clade consists of taxa from Hyalospora, Melampsoridium and Pucciniastrum and Milesinaceae includes Milesina. The genera Ravenelia, Kernkampella clustered in a Raveneliaceae clade and Maravalia formed a distinct lineage. While Nyssopsora belonging to Uredinineae incertae sedis formed a different lineage. The Tranzschelia includes Tranzschelia and Leucotelium and Ochropsoraceae comprises Ochropsora. The Phakopsoraceae includes Cerotelium and Phakopsora in a monophyletic clade. The Crossopsoraceae clade comprises Angiopsora, Crossopsora and Kweilingia. The Phragmidiaceae includes taxa from Phragmidium in monophyletic clade with significant support from ML 94\%. The Gymnosporangiaceae comprised Gymnosporangium in a monophyletic clade with significant support from ML 96\%. The Pucciniaceae includes polyphyletic taxa from Puccinia and Uromyces.

\section{Notes of Indian rust genera}

After going through the available literature on Indian rust fungi, it is now clear that identification of these fungi has primarily been based on morphological characters. Few studies are 
reported to use modern tools and molecular based techniques (specifically DNA-based) in their taxonomy. But wth the use of all modern molecular methdologies, a number of alterations in existing system of classification of rust fungi has been proposed by Cummins \& Hiratsuka (2003). Number of changes as proposed recently in classification of rust fungi (Aime 2006, Aime \& McTaggart 2020), has led to the introcution of many new families as well as transfer of many genera and species. Keeping in view all the proposed changes, this outline of Indian Pucciniales incorporates updated changes. Of the 18 families of rust fungi, 16 are reported from India, consists 69 genera and 640 species. Most of the genera and species of Indian collections still required DNA based identification. Therefore, a brief description of each rust family along with total number of genera as well as species reported from India and their host families are provided in this section. In addition, a brief note, where further studies on Indian collections are urgently required to resolve their taxonomic uncertainty is also provided.

Phylum - Basidiomycota R. T. Moore, Bot. Mar.23: 371 (1980).

Synonyms - Basidiomycota Bold, Morph. Pl.: 7, 198 (1958), nomen invalidum

Basidiomycota is the second largest phyla of kingdom Fungi which shares $97 \%$ of all fungal species along with phylum Ascomycota (Wijayawardene et al. 2017, 2018, 2020, Niskanen et al. 2018). The fungi included in Basidiomycota possess basidia as meiosporocysts in the sexual life stage. The hyphae appeared to have single-layered wall (which actually is multi-layered) are divided by septa into mononucleate, binucleate, or multinucleate segments. The septal pore is generally closed, however, in some cases barrel-like thickening is present on both sides. Chemotaxonomy, formation of urease, siderochromes, and the type of ubiquinone system also differentiate basidiomycetous fungi from ascomycetes. In addition, the guanine-cytosine content of the total DNA exceeds $50 \%$ in basidiomycetous species. The updated outline of Basidiomycota includes four subphyla, 18 classes, 68 orders, 241 families, 1928 genera and 41270 species, of which rust fungi are included in subphylum Pucciniomycotina (He et al. 2019, Wijayawardene et al. 2020).

Subphylum: Pucciniomycotina R. Bauer, Begerow, J.P. Samp., M. Weiß \& Oberw., Mycol. Progr.5: 45 (2006).

Equivalent to Urediniomycetes (Swann \& Taylor 1995b, Kirk et al. 2001, Swann et al. 2001).

Pucciniomycotina is a diverse group of fungi, including rusts, yeasts, smut-like and jelly-like fungi. It is the sister to the Ustilaginomycotina and Agaricomycotina, forming the basal lineage of Basidiomycota. Species of Pucciniomycotina studied so far lack dolipores (septal pore swellings) and septal pore caps. Absence of the predominant cell wall sugar, mannose (Prillinger et al. 1993) and disc like spindle pole bodies (McLaughlin et al. 1995, Wells 1994), distinguishes them from most other Basidiomycota. These fungi show very simple to complex life cycle, considered as most complex organisms (Lutz et al. 2004). Most described species are predominantly phytopathogens but also include asymptomatic members. Subphylum Pucciniomycotina is estimated to have 10 classes, 22 orders, 49 families, 270 genera, and 8653 species with rust fungi placed in class Pucciniomycetes (He et al. 2019, Wijayawardene et al. 2020).

Class: Pucciniomycetes R. Bauer, Begerow, J.P. Samp., M. Weiss \& Oberw., Mycol. Progr. 5: 48 (2006).

Equivalent to Urediniomycetidae (Swann et al. 2001).

Pucciniomycetes is a diverse class of subphylum Pucciniomycotina. It is one of the major classes of basidiomycete fungi containing about 8000 species (Kirk et al. 2008). Based on rDNA phylogenetic studies, rust fungi and their closest relatives in Pucciniomycetes are reported to have ambiguous phylogenetic positions within the Pucciniomycotina (Aime et al. 2006). All species of Pucciniomycetes are dikaryotic (containing two haploid nuclei per cell) except for Septobasidiales, which are monokaryotic (containing a single haploid nucleus per cell). Lack of clamp connections in their hyphae (Bauer et al. 2006) is another characteristic feature of these fungi. Production of asexual spores, especially among rusts, is often well developed (Bruckart et al. 2010). Sexual reproduction takes place via the formation of basidiospores. This class contains 5 orders, 20 families, 180 genera, 
and 8016 species. Majority of the fungal species in Pucciniomycetes are parasitic in nature. Pucciniales is the most species-rich group of the Pucciniomycetes with over $95 \%$ of the species and $75 \%$ of the genera placed in this order, the plant parasitic rust fungi.

Order: Pucciniales Clem. \& Shear, Gen. Fungi (2nd edn): 147 (1931).

Equivalent to Uredinales.

Exemplar genera: Puccinia Pers. 1801, Uromyces (Link) Unger 1832.

The Pucciniales is one of the largest and major orders in Basidiomycota (class Pucciniomycetes). This order mainly contains many important plant pathogens popularly known as rusts. These fungi are obligate plant parasites occurring on ferns, gymnosperms and angiosperms. They have been studied in detailed as many of the most devastating plant diseases in agricultural crops are caused by the members of Pucciniales. Morphologically, the species of Pucciniales are characterized by their rusty appearance on infected host parts such as leaves, petioles, tender shoots, stems, fruits, etc. and named for the typically rusty coloration of their urediniospores. These obligate parasites have highly complex life cycles with up to five spore stages and two unrelated hosts (Cummins \& Hiratsuka 2003). Of 7800 described species, Pucciniales constitutes $25 \%$ of all known species in Basidiomycota and ca. 8\% of all described Fungi (Kirk et al. 2008). The Pucciniales is estimated to have 15 families, ca. 150 genera and ca.7, 800 species, and is considered as the most speciose order of fungi (Kirk et al. 2008, Wijayawardene et al. (2020). Recently, a higher-rank classification for rust fungi, with notes on genera was provided by Aime \& McTaggart (2020). They have proposed four new suborders and seven new families based on the evaluation of $80 \%$ of accepted genera including type species wherever possible, and three DNA loci. As per this classification, Pucciniales now comprises seven suborders as Araucariomycetineae, Melampsorineae, Mikronegeriineae, Raveneliineae, Rogerpetersoniineae, Skierkineae, and Uredinineae. There are now 18 families Araucariomycetaceae, Coleosporiaceae, Crossopsoraceae, Gymnosporangiaceae, Melampsoraceae, Milesinaceae, Ochropsoraceae, Phakopsoraceae, Phragmidiaceae, Pileolariaceae, Pucciniaceae, Pucciniastraceae, Raveneliaceae, Rogerpetersoniaceae, Skierkaceae, Sphaerophragmiaceae, Tranzscheliaceae, and Zaghouaniaceae (Aime \& McTaggart 2020). Therefore, we followed Aime \& McTaggart (2020) to present updated information on genera and species in each family. The numbers of genera and species of rust fungi were presented as per Wijayawardene et al. (2020). The distinct characteristics of various spore stages (telia, teliospores, uredinia, uredinospores, aecia, aeciospores, spermogonia and basidia) for each family are summarized in this section. The notes on rust families reported from India are described here in this section of manuscript.

Coleosporiaceae Dietel, In: Engler \& Prantl, Nat. Pflanzenfam., Teil. I (Leipzig) 1: 548. 1900. emend. Aime \& McTaggart

Type genus - Coleosporium Lév., Ann. Sci. Nat. Bot. III, Ser. 8: 373.1847

The species of family Coleosporiaceae bear large, bladder-like aecia covered with welldeveloped peridium and have catenulate, verrucose aeciospores. Uredinia with rudimentary peridium or none and urediniospores are formed in chain with verrucose echinulate ornamentation on surface. The germ pores are mostly obscure and scattered. The teliospores are formed one by one (catenulate, pseudocatenulate or in a single layer) in erumpent, hard, waxy or gelatinous, pulvinate or columnar telia under the epidermis of the host plant. The teliospores are generally unicellular, thin walled and sessile with non-differentiated germ pores. The spermogonia are of Group I (type 2 or 3) (but Group II, type 9 in Cronartium). Most of the species are heteroecious and macrocyclic, with aecial stage on needle, buds and cones of conifers (Cummins \& Hiratsuka 2003, Aime \& McTaggart 2020).

Genera reported in India - Coleosporium (19), Chrysomyxa (7), Cronartium (4), Goplana (1), Stakmania (1) total 32 species.

Host families - Asteraceae, Campanulaceae, Ericaceae, Gentianaceae, Grossulariaceae, Lamiaceae, Lauraceae, Moraceae, Phyllanthaceae, Pinaceae, Ranunculaceae. 


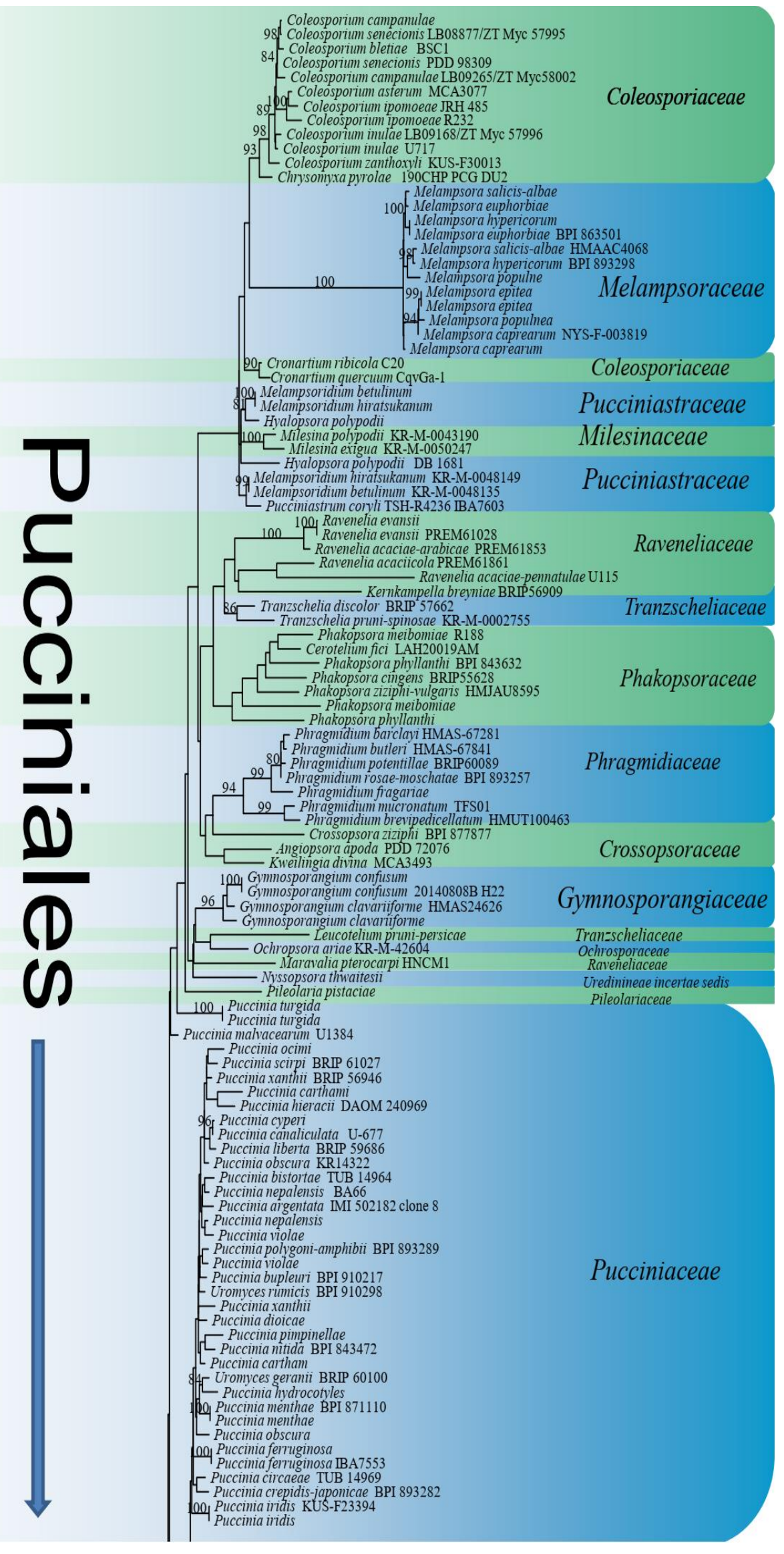

Figure 1 - Maximum likelihood phylogeny of Indian rust fungi based on two concatenated loci (LSU and ITS) of taxa from Pucciniales. Bootstrap support values for maximum likelihood equal to or 
greater than $70 \%$ are given above each branch respectively. Outgroup taxon is Taphrina pruni CBS 358.35 .

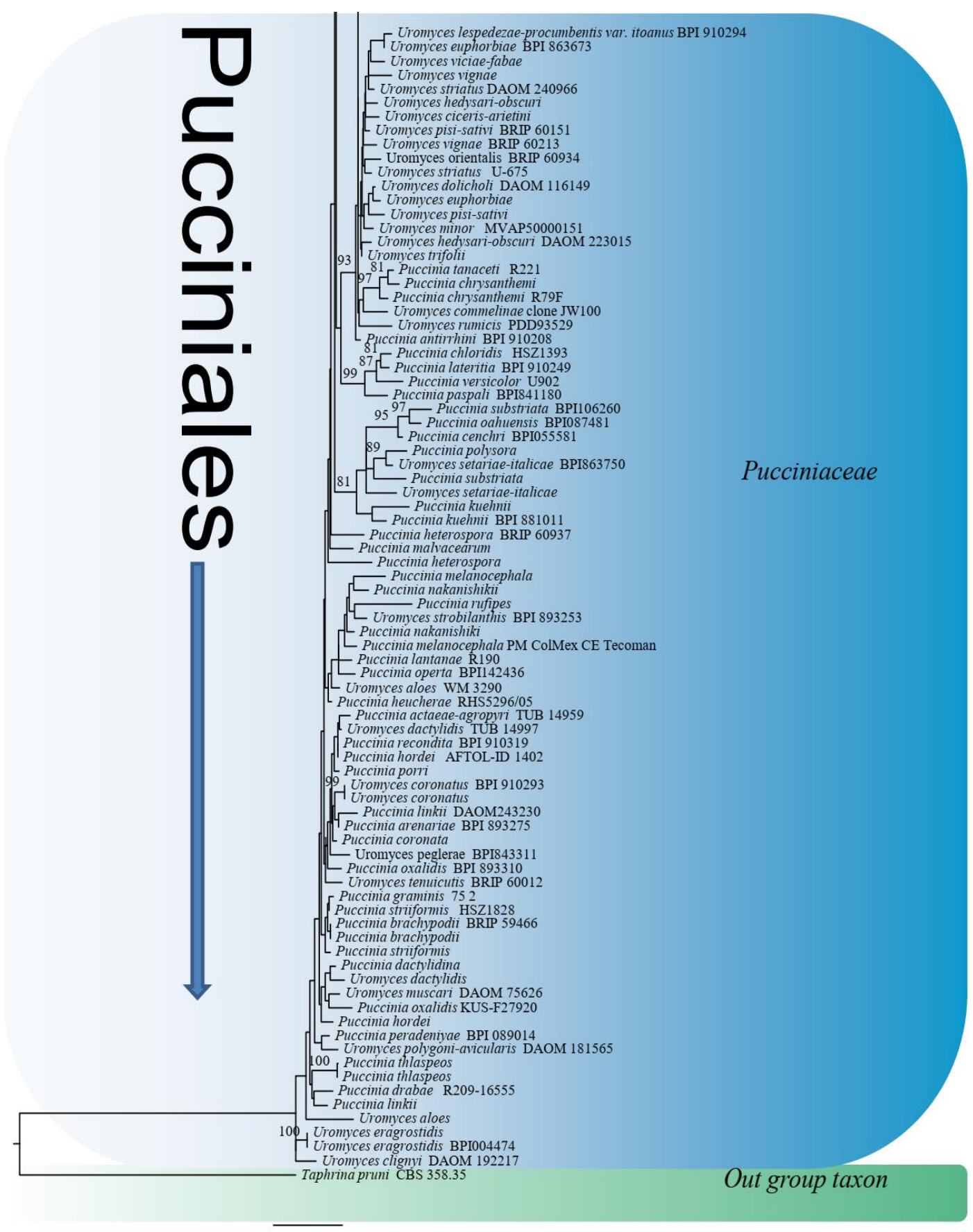

0.2

Figure 1 - Continued.

Notes - Two species of rust genus Stakmania (S. formosana and S. indica) were reported from India. Among the two, S. formosana Syd. \& P. Syd. has been transferred to Phakopsora formosana while, S. indica Kamat \& Sathe remains unchanged (Index Fungorum 2020). Hence, this species has been retained in this family.

Crossopsoraceae Aime \& McTaggart, In: Fungal Systematics and Evolution 7: 21-47. 2020

Type genus - Crossopsora Syd. \& P. Syd., Annls mycol. 16(3/6): 243. 1919. 
Crossopsoraceae is characterized by producing Group VI (type 7) spermogonia and aecidiumtype aecia wherever known. Uredinia are of malupa-type and usually paraphysate. The teliospores produced by these fungi are 1-celled, compact and often produced in catenulate chains of a few to many cells. These spores germinate externally, with or without dormancy. The fungi in this family are mostly identified from the sporothallus (uredinial, and telial) stages. The genus Neophysopella in this family is macrocyclic and heteroecious in nature (Aime \& McTaggart 2020) and considered the same for other genus/species also. A total of 7 genera have been included in this family, of which, six genera being reported from India.

Genera reported in India - Angiopsora (2), Crossopsora (3), Dasturella (3), Kweilingia (2), Neophysopella (2), Physopella (2); total 14 species.

Host families - Poaceae, Rhamnaceae Verbenaceae.

Notes - Two Indian records of the genus Physopella i.e. P. artocarpi (Berk. \& Broome) Arthur and P. vernoniae (T.S. Ramakr.) Ramachar \& Bhagyan are solely based on morphs. Since the genus Physopella has now been changed to Neophysopella, these two Indian records are still unchanged (Index Fungorum 2020). Hence, we retained this species in this family and proposed DNA based studies to resolve its taxonomic position.

Gymnosporangiaceae P. Zhou \& L. Cai, Persoonia 45: 79. 2020. emend. Aime \& McTaggart

Type genus - Gymnosporangium R. Hedw. ex DC., In: Lamarck \& de Candolle, Fl. franç., Edn 3 (Paris) 2: 216. 1805.

The family Gymnosporangiaceae consists of Group V (type 4) Spermogonia, bounded with well developed peripheral flexuous hyphae. Aecia Roestelia-type, subepidermal, with welldeveloped peridia (Gymnosporangium) or less frequently aecidium-type (Gymnotelium). Aeciospores catenulate, with intercalary cells. Urediniospores borne singly on pedicels in Uredotype, subepidermal Uredinia. Telia subepidermal, erumpent consists mostly 2-celled teliospores, borne singly on gelatinising pedicels without dormancy via external basidia. Life cycle mostly demicyclic and heteroecious (Zhao et al. 2020, Aime \& McTaggart 2020).

Genera reported in India - Peridiopsora (1) Gymnosporangium (3) Roestelia (3)

Host families - Boraginaceae, Cupressaceae, Rosaceae.

Notes - Based on puccinioid character of 2-celled, pedicellate teliospores, the genus name Gymnosporangium has been conserved against the older name Roestelia Rebent. (Aime et al. 2018b). Hence, the genus Roestelia has now been transferred to Gymnosporangium. Because of lack of molecular data, Indian records of this genus reported as Roestelia distorta (Arthur \& Cummins) F. Kern, Roestelia cunninghamianum (Barclay) F. Kern and Roestelia patula (Syd. \& P. Syd.) F. Kern are still unchanged (Index Fungorum 2020).

Melampsoraceae Dietel, in Engler \& Prantl, Nat. Pflanzenfam., Teil. I (Leipzig) 1: 38. 1897.

Figs 2, 7, 9

Type genus - Melampsora Castagne, Obs. Plantes Acotylédonées Fam. Urédinié 2: 18. 1843.

Melampsoraceae is characterized by aecia without peridium or rudimentary if present with catenulate and verrucose aeciospores. Uredinia contain abundant paraphyses (sometimes rudimentary peridium also) and echinulate urediniospores with scattered or bizonate germ pores and borne singly. Telia are embedded (subepidermal or rarely subcuticular) containing unicellular, sessile, pigmented teliospores with one germ pore. Germination external or semi-external (Ceropsora). The basidium is external and spermogonia are of Group I (Type 2 or 3 ). These fungi are mostly macrocyclic and inhabit two different unrelated hosts or same host to produce all spore stages. Most of the species are heteroecious and macrocyclic; however, the species of Ceropsora are microcyclic. Total 100 species of Melampsora have been reported globally (He et al. 2019, Wijayawardene et al. 2020), of which only 17 species have been reported so far in India.

Genera reported in India - Ceropsora (1), Melampsora (17); total 18 species.

Host families - Euphorbiaceae, Hypericaceae, Linaceae, Pinaceae, Salicaceae. 
Notes - The type species of the genus Ceropsora (C. picea) infecting Picea sp. was reported from India (Bakshi \& Singh 1960). It was placed in family Coleosporiaceae (Cummins \& Hiratsuka 2003, Wijayawardene et al. 2020). The telia of two species of Ceropsora viz., C. picea and C. weirii contain some thin-walled sterile cells on the sides (been interpreted as remnants of a peridermium). Teliospores of the genus are subtended by adherent crusts of sterile basal cells in the beginning that separate at dispersal stage (Bakshi \& Singh 1960, Crane et al. 2000). Aime \& McTaggart (2020) proposed the inclusion of the genus Ceropsora in to this family.

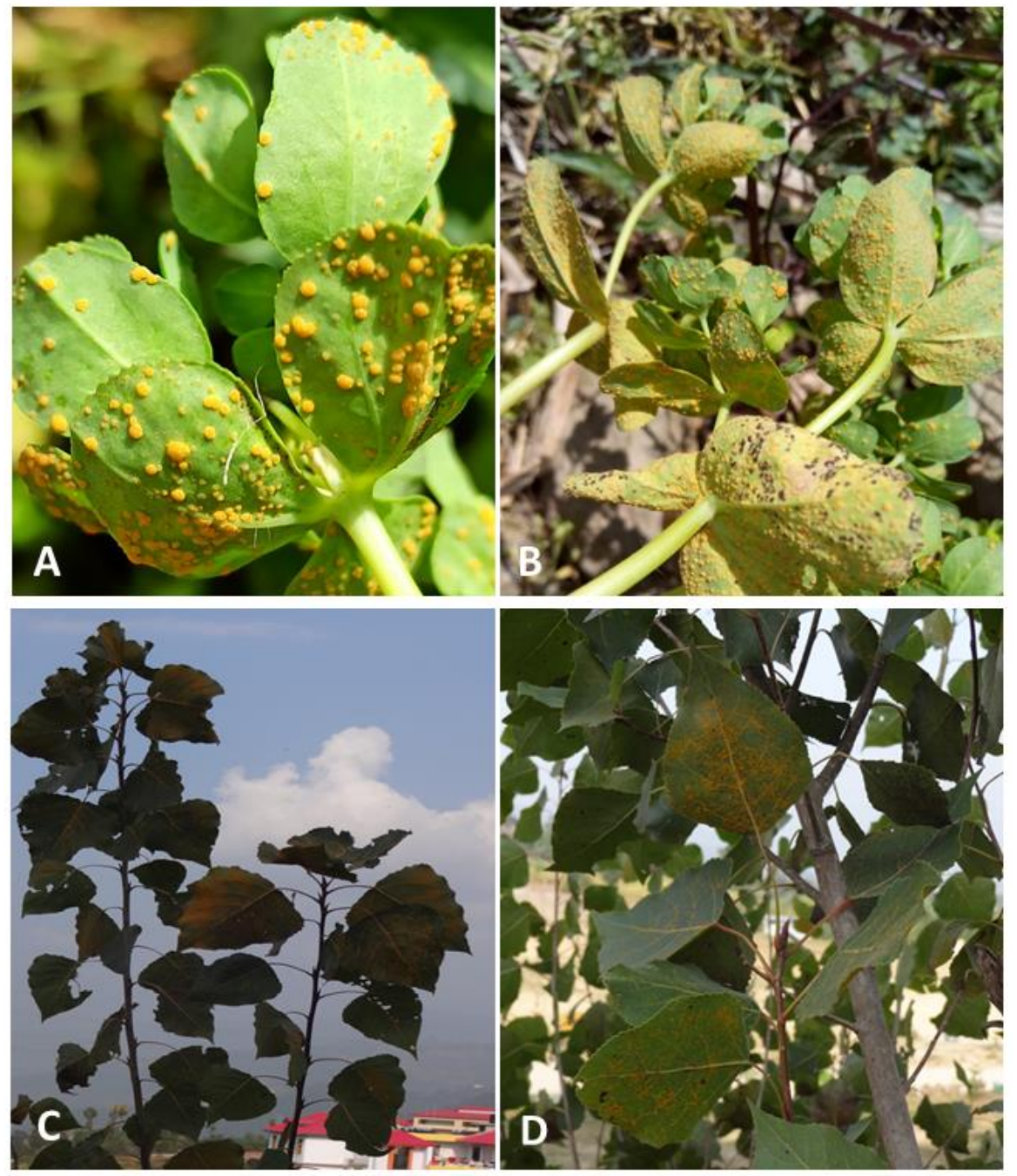

Figure 2 - Rust fungi Melampsora sp. A-B Euphorbia helioscopia. C-D Populus sp.

Milesinaceae Aime \& McTaggart, In: Fungal Systematics and Evolution 7: 21-47. 2020

Type genus - Milesina Magnus, Ber. Deutsch. Bot. Ges. 27: 325. 1909.

Two important characters, production of colourless urediniospores in species that infect ferns and production of aecia (milesia-type) in species that infect Ericaceae differentiate this family from Melampsorineae. The important characters of this family include production of Group I (mostly type 1, also type 2 and 3) Spermogonia and colourless sori. Although urediniospores of Naohidemyces are orange in colour. The presence of peridermium-type aecia (milesia-type in Naohidemyces); milesiatype uredinia; 1- to many-celled, barely differentiated, sometimes laterally adherent teliospores with dormant germination are found. These fungi are mostly macrocyclic and heteroecious in nature. They produce sporothalli on ferns (except Naohidemyces on Ericaceae), and gametothalli on Pinaceae.

Genera reported in India - Milesina (3), Uredinopsis (2); total 5 species

Host families - Dryopteridaceae, Pteridaceae. 
Note - This is newly proposed family by Aime \& McTaggart (2020). Out of 4 genera included in this family (Aime \& McTaggart 2020), two genera namely, Milesina and Uredinopsis are reported from India. Aime et al. (2018) recommended the protection of the name Milesina Magnus over Milesia F.B. White, but they do not found the type species of both the genera congeneric and recommended retaining both the genera. In India, the molecular studes on Milesina and Uredinopsis are also required to demonstrate their correct taxonomic position.

Ochropsoraceae (Arthur) Aime \& McTaggart, In: Fungal Systematics and Evolution 7: 21-47. 2020 Type genus - Ochropsora Dietel, Ber. Dtsch. Bot. Ges. 13: 401. 1895.

The family Ochropsoraceae contains Group VI (type 7) spermogonia. Aecia are of aecidiumtype, these states systemic in nature and overwintering as mycelium. Uredinia are of malupa-type. The genera of this family produce 1-cell deep telia forming crusts, which are at first subepidermal, then erumpent. Teliospores germinate without dormancy, either internally (Ochropsora) or externally (Aplopsora). These fungi are macrocyclic and heteroecious in nature.

Genera reported in India - Ochropsora (1); total 1 species

Host families - Ranunculaceae.

Note - Aime \& McTaggart (2020) proposed Ochropsoraceae as new family. The rust genera Aplopsora and Ochropsora included in this family were previously treated within the Chaconiaceae (Cummins \& Hiratsuka 2003). Of these, Ochropsora (Ochropsora ariae (Fuckel) Ramsb.) was also reported from India. However, only morphotaxonomic studies are available for this record, and molecular studies can be helpful to establish the correct taxonomic position.

Phakopsoraceae Cummins \& Y. Hirats., Illustr. Gen. Rust Fungi, rev. Edn (St. Paul): 13. 1983. emend. Aime \& McTaggart

Type genus - Phakopsora Dietel, Ber. Deutsch. Bot. Ges. 13: 333. 1895.

The genera of Phakopsoraceae show both heteroecious and autoecious (species of Bubakia, Masseeëlla and Nothoravenelia) mode of life cycle on various hosts but not host restricted. Spermogonia are of Group IV (type 7). The aecia are caeoma-type (some Masseeëlla with aecidiumtype) with aeciospores either verrucose or echinulate, borne singly or in chains. Uredinia lecythea or uredo-type consisting echinulate urediniospores, mostly borne singly (catenulate in Arthuria) with scattered germ pores or obscure. Both erumpent and embedded types of telia are found which contain 1-celled, sessile, catenulate or irregularly arranged teliospores with mostly 1 germ pore in each cell The majority of Phakopsora and Uredopeltis species are only known from the sporothallus (uredinial, and telial) stages (Cummins \& Hiratsuka 2003, Aime \& McTaggart 2020). This family comprises 15 genera and 209 species (Wijayawardene et al. 2020). Aime \& McTaggart (2020) in recent treatment included 12 genera in this family, 10 being reported from India.

Genera reported in India - Arthuria (2), Bubakia (1), Cerotelium (7), Macabuna (1), Monosporidium (3), Masseeëlla (6), Phakopsora (25), Phragmidiella (3), Pucciniostele (1), Uredopeltis (1); total 50 species.

Host families - Bignoniaceae, Burseraceae, Combretaceae, Euphorbiaceae, Fabaceae, Phyllanthaceae, Putranjivaceae, Rhamnaceae, Rubiaceae, Salicaceae, Saxifragaceae and Vitaceae.

Notes - The rust genus Bubakia has now been transferred to Phakopsora (Index Fungorum 2021). It is often treated as a synonym of Phakopsora (Cummins \& Hiratsuka 2003). But, Aime \& McTaggart (2020) found a distinct lineage of the Bubakia argentinensis within Phakopsoraceae and similar characteristics and hosts with type, B. crotonis. Hence, they proposed Bubakia for these species. However, the only India record of Bubakia indica T.S. Ramakr. \& K. Ramakr. still requires molecular studes, as it was identified based on morphology only. The genus Masseeëlla is also placed within this family (Aime \& McTaggart 2020), although previously treated as incertae sedis within Pucciniales (Cummins \& Hiratsuka 2003).

Phragmidiaceae Corda Icon. fung. (Prague) 1: 6. 1837

Figs 3, 9

Type genus - Phragmidium Link, Mag. Ges. Naturfr. Freunde Berlin 7: 30. 1816. 
Phragmidiaceae is characterized by forming spermogonia Group IV (type 6, 8, 10 or 11). The aecia variable, caeoma-, petersonia- or uredo-type and are formed with or without peridium. Aeciospores are verrucose or echninulate aeciospores borne in chains or singly on pedicels with few or no paraphyses. Uredinia lecythea or uredo-type, thin-walled, with incurved paraphyses consisting of echninulate urediniospores borne singly and have scattered germ pores. Telia erumpent, with or without paraphyses, with teliospores born on short pedicels, 3- to several cells (by transverse septa) with 1 or more apical germ pore in each cell. Basidium is external. Life cycle is mainly of autoecious type. Wijayawardene et al. (2020) documented 13 genera and 178 species in this family; however, Aime \& McTaggart (2020) included only 9 genera.

Genera reported in India - Hamaspora (2), Kuehneola (6), Phragmidium (18), Trachyspora (1); total 27 species.

Host families - Poaceae, Rosaceae.

Notes - Of the 9 genera included in this family, only 4 genera and 27 species have been reported in India. Phragmidiaceae species are confined almost exclusively to the Rosoideae subfamily of Rosaceae (Aime 2006).
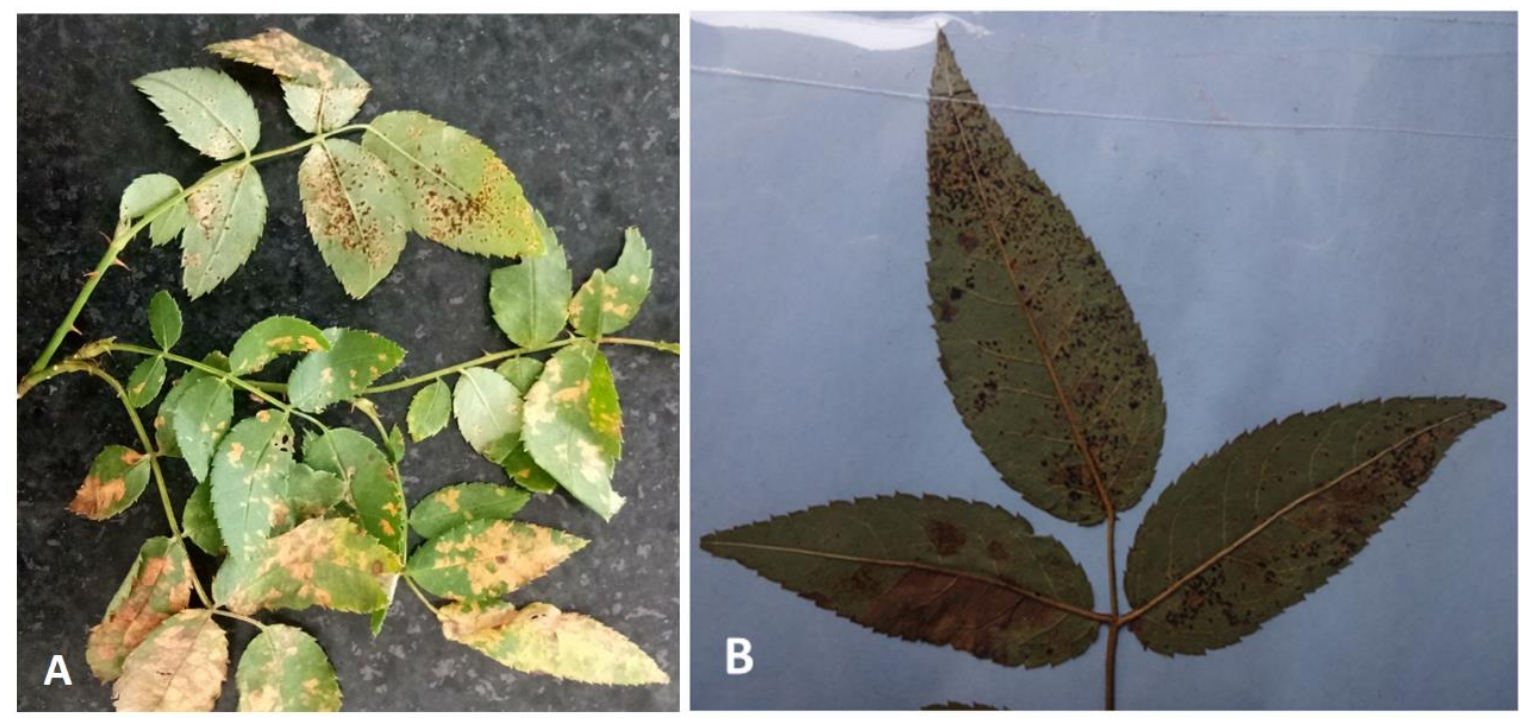

Figure 3 - A-B Phragmidium sp. on Rosa sp.

Pileolariaceae (Arthur) Cummins \& Y. Hirats., llustr. Gen. Rust Fungi, rev. Edn (St. Paul): 14. 1983. emend. Aime \& McTaggart

Figs 4A, 9

Type genus - Pileolaria Castagne, Obs. Plantes Acotylédonées Fam. Urédinées 1: 22.1842.

The genera of the family are characterized by forming spermogonia Group VI (type 7). Aecia (uredo-type) with or without peridium contains reticulate, ridged, verrucose, spirally marked aeciospores borne singly and germ pores zonate. Uredia (uredo-type) are similar to aecia except not accompanied by spermogonia. Telia erumpent and having unicellular teliospores 1-celled, borne one or few on each pedicel with one germ pore per spore. Basidium is external. Species are mostly macrocyclic and autoecious. The genera of this family mostly inhabit members of Anacardiaceae (Cummins \& Hiratsuka 2003, Aime \& McTaggart 2020).

Genera reported in India - Pileolaria (2); total 2 species.

Host family - Anacardiaceae.

Notes - Four genera and 43 species have been documented in this family (Wijayawardene et al. 2020), of which two genera, Pileolaria and Skierka were reported from India. However, Aime \& McTaggart (2020) included only the genus i.e. Pileolaria and proposed the placement of Skierka within another family. A total 25 species of Pileolaria are recorded worldwide (Index Fungorum 2021). The two Indian records (P. indica Syd. and P. pistaciae F.L. Tai \& C.T. Wei) require further molecular studies. 



Figure 4 - Rust fungi. A Pileolaria sp. on Pistacia sp. B Skierka sp. on Pistacia sp.

Pucciniastraceae Gäum. ex Leppik, Ann. bot. fenn. 9: 139. 1972. emend. Aime \& McTaggart

Type genus - Pucciniastrum G.H. Otth, Mitt. Naturforsch. Ges. Bern 1861: 71. 1861.

Pucciniastraceae members are heteroecious and mostly macrocyclic. Spermogonia and aecia produced on conifers; uredinia and telia often on ferns. Spermogonia are of Group I (type 2 or 3). Aecia with well developed peridium and contain catenulate aeciospores. Uredinia are formed with cellular peridium and ostiolar opening and contain pedicellate or non pedicellate, echinulate urediniospores, borne singly and possess obscure, scattered or bizonate germ pores. Telia are not well differentiated i.e. either subepidermal or intradermal, not erumpent, composed of unicellular or multicellular, sessile teliospores having obscure or 1 germ pore per cell. Basidia are external (Cummins \& Hiratsuka 2003, Aime \& McTaggart 2020). The family was reported to contain 10 genera and 210 species (Wijayawardene et al. 2020). The recent higher rank classification for rust fungi proposed the inclusion of only six genera in this family (Aime \& McTaggart 2020), of which four were recorded from India.

Genera reported in India - Hyalopsora (2), Melampsoridium (4), Peridermium (7), Pucciniastrum (6); total 19 species.

Host families - Betulaceae, Celastraceae, Coriariaceae Magnoliaceae, Phyllanthaceae, Pinaceae, Pteridaceae, Rosaceae, Sapindaceae.

Notes - As per the proposal to conserve the name of rust fungi (Aime et al. 2018), the sexual name Melampsorella has been conserved over Peridermium. However, the Indian records of Peridermium are devoid of any molecular studies and are still unchanged (Index Fungorum 2021).

Pucciniaceae Chevall., Fl. gén. env. Paris (Paris) 1: 413. 1826. emend. Aime \& McTaggart

Type genus - Puccinia Pers., Synopsis methodica fungorum: 225. 1801.

Figs 5, 6, 7, 8

Pucciniaceae is a largest family that infects nearly all the major angiospermous orders. They are destructive phytopathogens infecting mainly cereals such as wheat. Spermogonia belong to Group V (type 4). Aecidia are with or without a peridium and have verrucose aeciospores borne singly, or in chain (catenulate). Uredinia with palisade like peridium contain echinulate urediniospores borne singly and have many germ pores. The most important distinguishing character is that these fungi having stalked teliospores borne singly or united in telia with or without paraphyses. In some cases, palisade like peridium or separation of telia into locules by stromatoid paraphyses is also observed. 
Teliospores are mostly 1 or 2 (rarely more) celled with one germ pore in each cell and germination mostly by external basidium (sometimes internal). The genera are mostly heteroecious exhibiting four spore stages usually upon two or more distinct hosts followed by an independent promycelial stage upon germination of the teliospores. The largest two genera are Puccinia, with two-celled teliospores, and Uromyces where they are one-celled. This is the most speciose family of the Pucciniales, and contains 21 genera and over 4961 species (Wijayawardene et al. 2020). Aime \& McTaggart (2020) proposed the inclusion of 23 genera and 9 more genera likely to include in this family. Total 12 genera with 393 species have been recorded in India.

Genera reported in India - Caeoma (4), Chrysocelis (1), Corbulopsora (1), Endophyllum (8), Gambleola (1), Hapalophragmium (4), Kernella (1), Puccinia (279), Pucciniosira (1), Ramakrishnania (1), Trochodium (2), Uromyces (89); total 393 species.

Host families - Acanthaceae, Apiaceae, Asteraceae, Berberidaceae, Boraginaceae, Celastraceae, Convolvulaceae, Cyperaceae, Elaeagnaceae, Euphorbiaceae, Fabaceae, Lamiaceae, Lauraceae, Liliaceae, Loranthaceae, Malvaceae, Orchidaceae, Pinaceae, Plantaginaceae, Poaceae, Polygonaceae, Ranunculaceae, Rubiaceae, Saxifragaceae, Schisandraceae, Solanaceae.

Notes - The genus Caeoma, as typified by $C$. berberidis, is a synonym of Puccinia (Aime et al. 2018). One of its species $C$. torreyae is presented as basionym of Rogerpetersonia torreyae (Bonar) Aime \& McTaggart (Aime \& McTaggart 2020) based on broad molecular assessment. Similarly, the genus Trochodium has now been transferred to Uromyces. The types of two genera, Gambleola and Ramakrishnania are of Indian origin and identified mainly based on mophotaxonomic characters. The Indian records for all three genera are still unchanged (Index fungorum 2021) and required investigation at molecular level.

Raveneliaceae Leppik, Ann. bot. fenn. 9: 139. 1972. emend. Aime \& McTaggart

Fig. 9

Type genus - Ravenelia Berk., Gard. Chron. 13:132. 1853.

Raveneliaceae consists Group VI (type 5,7) spermogonia. The genera of this family produce typically uredo- (rarely aecidium-, caeoma-, or lecythea-) type aecia with or without peridium and paraphyses. Aeciospores are pedicellate and echinulate or verrucose borne singly or in chain. Uredinia are of subepidermal or erumpent, with or without paraphyses. These are similar to aecia while unknown in some cases. Urediniospores borne singly, mostly echinulate with several germ pores. Telia are erumpent with or without paraphyses contain pedicellate, vertically septate or vertically or radially arranged 1 - to many-celled teliospores ( 2 or more) on the top of pedicel, often subtended by hygroscopic crystals or with pedicel having apical cells. Each cell or spore contains one or two germ pores. Basidium is of external type. Members of this family have autoecious and macrocyclic type of life cycle mostly on Fabaceae or Rosaceae. Although 24 genera and 384 species have been documented within this family (Wijayawardene et al. 2020), Aime \& McTaggart (2020) proposed the inclusion of 16 genera and 16 more genera likely to include in this family. Total 10 genera with 61 species have been recorded in India.

Genera reported in India - Chaconia (1), Didymopsorella (1), Diorchidium (3), Gymnopuccinia (1), Kernkampella (5), Maravalia (11), Scopella (1), Olivea (3), Prospodium (2) Ravenelia (33); total 61 species.

Host families - Fabaceae, Oleaceae, Phyllanthaceae, Poaeae, Rubiaceae, Rutaceae.

Notes - The identification of Indian records in this family, Didymopsorella macrospora (Mundk. \& Thirum.) Thirum., Gymnopuccinia pulneyensis K. Ramakr. and Scopella dalbergiae (T.S. Ramakr. \& K. Ramakr.) Ragunathan \& K. Ramakr. is mainly based on their morphological caharcters. Similarly, the genus Scopella has now been transferred to Maravalia. However, its indian record Scopella dalbergiae (T.S. Ramakr. \& K. Ramakr.) Ragunathan \& K. Ramakr. is still unchanged. DNA sequence studies can be helpful to establish their correct taxonomic placement.

Skierkaceae (Arthur) Aime \& McTaggart, Fungal Systematics and Evolution 7: 21-47, 2020.

Type genus - Skierka Racib., Parasit. Alg. Pilze Javas (Jakarta) 2: 30. 1900. 
Skierkaceae is a newly introduced family by Aime \& McTaggart (2020), mainly characterized by subepidermal, periphysate, deep-seated spermogonia with convex hymenium. Aecia and uredinia uredo-type. The Uredia and telia (sporothalli sori) are deep-seated and subepidermal which differentailed these from all other rust fungi. Urediniospores and teliospores are single-celled produced on sporogenous cells through a narrow sorus opening. Before emenrgence, these spores leaving behind new spores on sporogenous cells from which they are detached. Teliospores strongly adherent, extruded in hair-like columns, germination external, without dormancy. These fungi possess autoecious and macrocyclic type of life cycle.

Genera reported in India - Skierka (3); total 3 species.

Host families - Anacardiaceae.

Notes - A total of 14 species of the genus Skierka have been reported so far (Index Fungorum 2021), three species reported from India too. Skierka himalayensis A.K. Gautam \& S. Avasthi was reported as new from India (Gautam \& Avasthi 2017c). But all three records are identified on based on morphological characters only and require molecular identification.

Sphaerophragmiaceae Cummins \& Y. Hirats., Illustr. Gen. Rust Fungi, rev. Edn (St. Paul): 15. 1983. emend. Aime \& McTaggart

Type genus - Sphaerophragmium Magnus Ber. dt. bot. Ges 9: 121. 1891.

Spermogonia are mostly lacking and unknown, Group V (type 4) in Sphenorchidium, if present. Aecia are aecidium-type and uredinia resembling aecia (lecythea-type in Sphenorchidium). Teliospores 2- to multicelled, pedicellate, globose to subglobose, with furcated or simple blunt wall projections, with one germ pore per cell borne in compact telia (Beenken \& Berndt 2010). In Austropuccinia, urediniospores with a smooth patch (tonsure) as comparison to echinulate or verrucose in other cases. Telia subepidermal to erumpent, cylindrical to ellipsoidal, with a rounded apex, 2-celled teliospores, constricted at the septum. Basidia are mostly external. Species are autoecious with variable life cycles. Wijayawardene et al. (2020) documented 2 genera and 25 species in this family, however, Aime \& McTaggart (2020) proposed the inclusion of 5 genera.

Genera reported in India - Sphaerophragmium (1); total 1 species.

Host families - Fabaceae.

Notes - Only single genus Sphaerophragmium with one species (S. acacia (Cooke) Magnus) was reported from India. The identification of this species is solely based on moprhological characters, and DNA based molecular studies are necessary.

Tranzscheliaceae (Arthur) Aime \& McTaggart, Fungal Systematics and Evolution 7: 21-47, 2020

Type genus - Tranzschelia Arthur, Rés. Sci. Congr. Int. Vienne: 340. 1906.

The rust fungi of family Tranzscheliaceae are mostly macrocyclic and heteroecious in nature. However, some microcyclic species may be found. The species consists of Group VI (type 7) spermogonia. Aecia are of aecidium type while, uredinia uredo-type. Teliospores produced cby theses fungi are 2-celled, pedicellate, produced from sterile basal cells.

Genera reported in India - Leucotelium (1), Tranzschelia (2); total 3 species

Host families - Rosaceae.

Notes - Tranzscheliaceae is proposed as new family by Aime \& McTaggart (2020), included two genera, Leucotelium and Tranzschelia. Both the genera were previously treated within Uropyxidaceae (Cummins \& Hiratsuka 2003). The Indian records of these genera still required DNA sequence based studies to establish their correct taxonomic position.

Zaghouaniaceae P. Syd. \& Syd., Monogr. Uredin. (Lipsiae) 3(3): 586. 1915. emend. Aime \& McTaggart

Type genus - Zaghouania Pat., Bull. Soc. mycol. Fr. 17: 187. 1901

The species of this family most often consists of deep seated and non-periphysate Group III (type 12) spermogonia. Aecia are mostly petersonia-type (without peridium or intercalary cells). Aeciospores are echinulate or verrucose; borne singly or in chain. The uredinia are generally 
produced without peridium (most often uredo-type) or weakly developed peridium in some cases and produce echinulate, singly borne ureniniospores. Telia are with or without paraphyses, produce teliospores germinating externally (internally in some cases) by apical growth without dormancy. These rust fungi have both autoecious and heteroecious mode of life cycle.

Genera reported in India - Cystopsora (1), Elateraceium (1), Hemileia (10), Zaghouania (1); total 13 species

Host families - Apocynaceae, Celastraceae, Oleaceae, Penaeaceae, Phyllanthaceae, Rubiaceae.

Notes - Aime \& McTaggart (2020) included 8 genera in this family, of which, four genera namely Cystopsora, Elateraceium, Hemileia, Zaghouania have been reported from India. The genus Cystopsora is now considered as synonym of Zaghouania (Aime \& McTaggart 2020). The identity of Indian record Cystopsora antidesmatis T.S. Ramakr. \& Sundaram is mainly based on morphological observations. DNA sequence based studies are required to provide its exact taxonomic position.

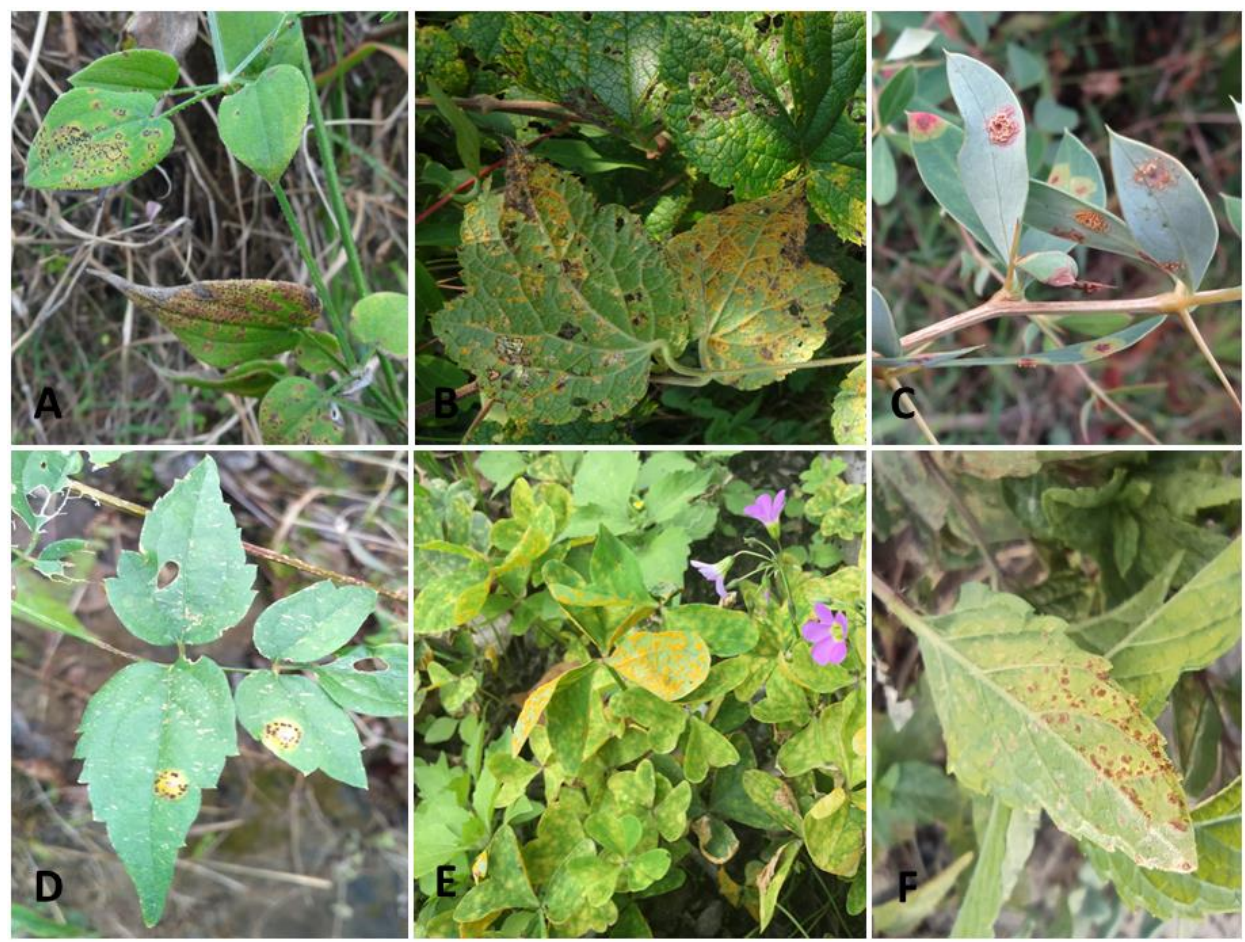

Figure 5 - Rust fungi Puccinia spp. A Rubia cordifolia. B Clematis sp. C Berberis sp. D Clematis sp. E Oxalis sp. F Mentha sp.

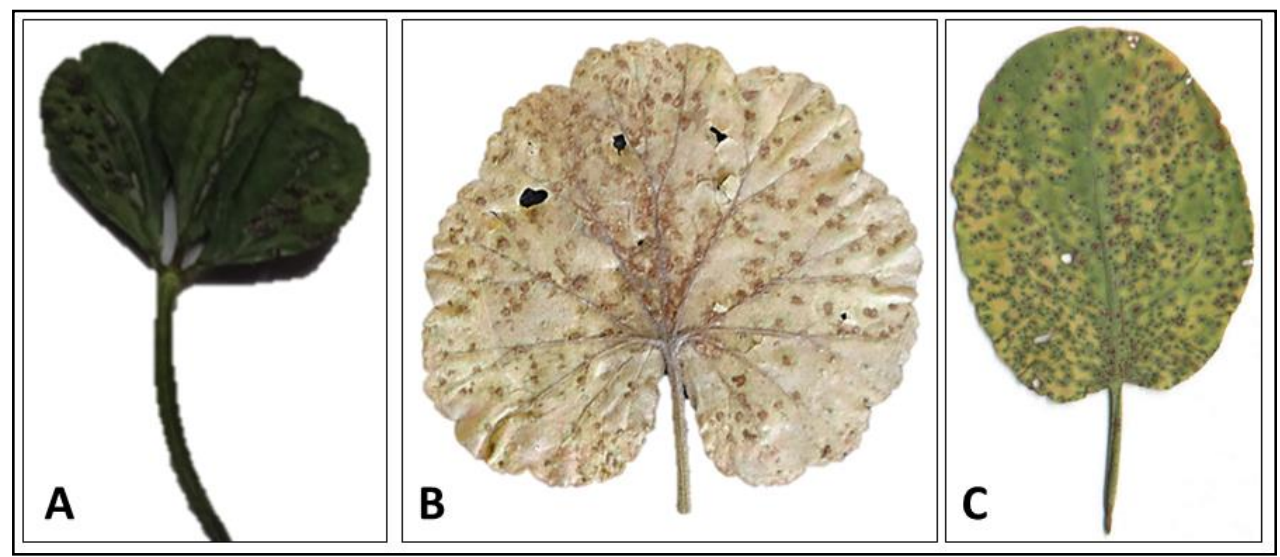

Figure 6 - Rust fungi Uromyces spp. A Trifolium sp. B Geranium sp. C Rumex sp. 



Figure 7 - Uredinospores. A Puccinia himachalensis on Clematis sp. B P. tiliaefolia on Grewia sp. C P. fagopyri on Fagopyrum sp. D P. menthae on Mentha sp. E P. Oxalidis on Oxalis sp. F P. flavipes on Duchesnea sp. G P. abrupta on Parthenium sp. H P. colletiana on Rubia sp. I Melampsora caprearum on Salix sp. J P. Nepalensis on Rumex sp. K Uredo sp. on Ehretia sp. L Melampsora populnea on Populus sp. Scale Bar $=10 \mu \mathrm{m}$. 

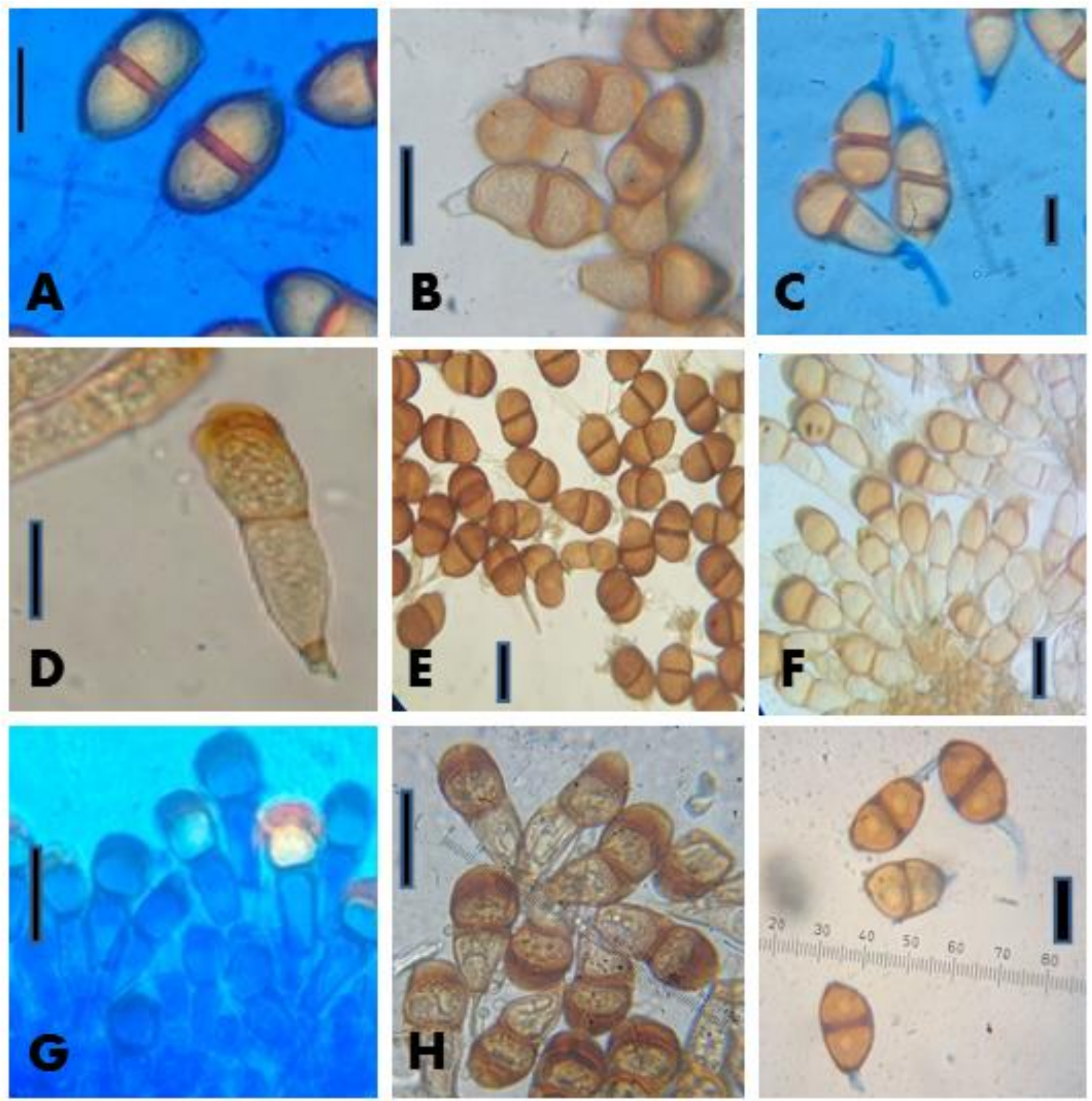

Figure 8 - Teliospores of Puccinia. A P. himachalensis on Clematis sp. B P. fagopyri on Fagopyrum sp. C P. tiliaefolia on Grewia sp. D P. agrostdis on Aquilegia sp. E P. Cynodontis on Cyanodon sp. F P. cynodontis on Cyanodon sp. G P. colletiana on Rubia sp. H P. colletiana on Rubia sp. I P. gouriana on Clematis sp. Scale Bar $=10 \mu \mathrm{m}$.

\section{Excluded and replaced names of rust fungi in India}

With the use of DNA sequence based techniques, the economically important rusts are relatively well explored, but not much attention has been paid to species infecting wild plants in general. The identification of most of the Indian rust fungi is largely based on morphological characters especially morphology of certain spore stages. Use of DNA sequence based studies along with morphotaxonomic characters has made identification and characterization of rust fungi more efficient and accurate. The names of many rust genera/species as reported in the cited publications have been replaced with new accepted names. Numbers of genera and species of Indian rust fungi have also been replaced with currently accepted name according to MycoBank (www.mycobank.org)/ and Species Fungorum (www.speciesfungorum.org) websites and this is indicated in Table 3. 


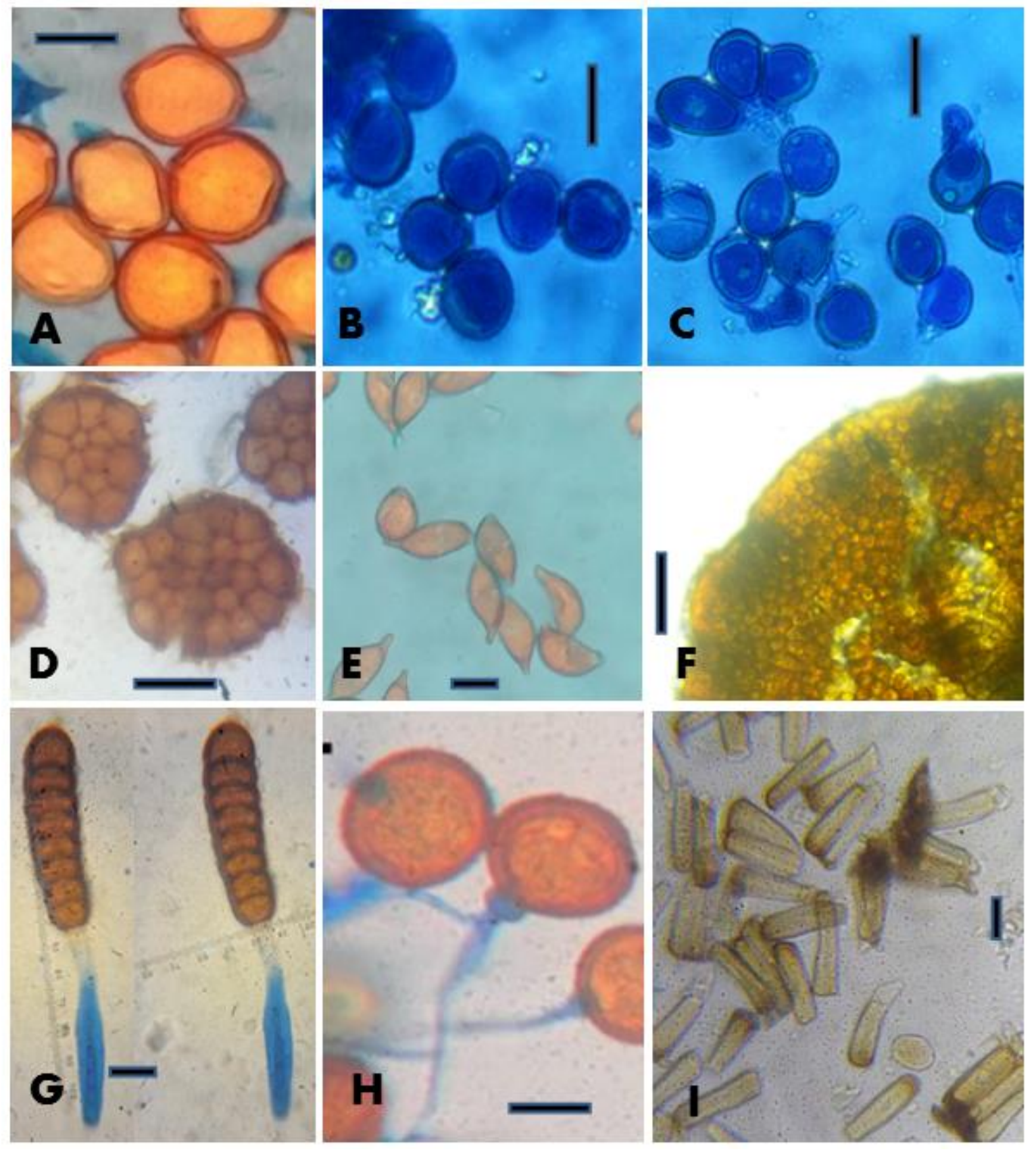

Figure 9 - Teliospores of rust fungi. A Uromyces on Trifolium sp. B Uromyces on Rumex sp. C Uromyces on Geranium sp. D Ravenelia on Pongamia sp. E Skierka on Pistacia sp. F Kweilingia on Bamusa sp. G Phragmidium on Rosa sp. H Pileolaria on Pistacia sp. I Melampsora on Euphorbia sp. Scale Bar $=10 \mu \mathrm{m}$.

Table 3 Excluded and replaced names of rust fungi in India. (Index Fungorum 2020, Mycobank 2020)

\begin{tabular}{|l|l|}
\hline Old Name & New Name \\
\hline Acervulopsora ichnocarpi (Barclay) Thirum. & Maravalia ichnocarpi (Barclay) Sathe \\
\hline Aecidium acanthospermi P.B. Chavan \& Bakare & Puccinia acanthospermi Henn. \\
\hline Aecidium asterum Schwein. & Puccinia asterum (Schwein.) F. Kern \\
\hline Aecidium barleriae M.A. Salam \& Ramachar & Aecidium salamii G.F. Laundon \\
\hline Aecidium berberidis Pers. ex J.F. Gmel. & Puccinia graminis Pers. \\
\hline Aecidium cassiae Bres. & Endophyllum cassiae (Bres.) F. Stevens \& Mendiola \\
\hline Aecidium cunninghamianum Barclay & Roestelia cunninghamianum (Barclay) F. Kern \\
\hline
\end{tabular}


Table 3 Continued.

\begin{tabular}{|c|c|}
\hline Old Name & New Name \\
\hline Aecidium elaeagni-latifoliae Petch & $\begin{array}{l}\text { Endophyllum elaeagni-latifoliae (Petch) Gokhale, } \\
\text { Thirum. \& Patel }\end{array}$ \\
\hline Aecidium kaernbachii Henn. & $\begin{array}{l}\text { Endophyllum kaernbachii (Henn.) F. Stevens \& } \\
\text { Mendiola }\end{array}$ \\
\hline Aecidium leucospermum DC. & Ochropsora ariae (Fuckel) Ramsb. \\
\hline Aecidium macowanianum Thüm. & Endophyllum macowanianum (Thüm.) Pole-Evans \\
\hline Aecidium meliosmae-myrianthae Henn. \& Shirai & $\begin{array}{l}\text { Neophysopella meliosmae-myrianthae (Henn. \& Shirai) } \\
\text { Jing X. Ji \& Kakish. }\end{array}$ \\
\hline Aecidium patulum Syd. \& P. Syd. & Roestelia patula (Syd. \& P. Syd.) F. Kern \\
\hline Aecidium plectranthi Barclay & Coleosporium plectranthi (Barclay) Sacc. \\
\hline Aecidium ranunculacearum DC. & Uromyces dactylidis G.H. Otth \\
\hline $\begin{array}{l}\text { Angiopsora ampelopsidis (Dietel \& P. Syd.) Thirum. } \\
\text { \& F. Kern }\end{array}$ & $\begin{array}{l}\text { Neophysopella ampelopsidis (Dietel \& P. Syd.) Jing X. } \\
\text { Ji \& Kakish. }\end{array}$ \\
\hline $\begin{array}{l}\text { Angiopsora elephantopodis (Hirats.) Mundk. \& } \\
\text { Thirum. }\end{array}$ & Phakopsora elephantopodis Hirats. \\
\hline Angiopsora meliosmae (Kusano) Thirum. \& F. Kern & $\begin{array}{l}\text { Neophysopella meliosmae (Kusano) Jing X. Ji \& } \\
\text { Kakish. }\end{array}$ \\
\hline Angiopsora vernoniae T.S. Ramakr. & $\begin{array}{l}\text { Physopella vernoniae (T.S. Ramakr.) Ramachar \& } \\
\text { Bhagyan. }\end{array}$ \\
\hline Bubakia cingens (Syd. \& P. Syd.) S. Ito & Phakopsora cingens (Syd. \& P. Syd.) Hirats. \\
\hline Catenulopsora flacourtiae Mundk. \& Thirum. & Kuehneola flacourtiae (Mundk. \& Thirum.) Thirum. \\
\hline Catenulopsora grewiae Mundk. \& Thirum. & Kuehneola grewiae (Mundk. \& Thirum.) Thirum. \\
\hline Catenulopsora ziziphi T.S. Ramakr. \& Subram. & Kuehneola ziziphi (T.S. Ramakr. \& Subram.) Thirum. \\
\hline Cerotelium fici (Castagne) Arthur & Puccinia fuirenicola Arthur \\
\hline Chnoopsora butleri Dietel, Syd. \& P. Syd. & $\begin{array}{l}\text { Chrysocelis butleri (Dietel, Syd. \& P. Syd.) G.F. } \\
\text { Laundon }\end{array}$ \\
\hline Chnoopsora sancti-johannis (Barclay) Dietel & Melampsora sancti-johannis Barclay \\
\hline Cystopsora oleae E.J. Butler & Zaghouania oleae (E.J. Butler) Cummins \\
\hline Dasturella divina (Syd.) Mundk. \& Khesw. & Kweilingia divina (Syd.) Buriticá \\
\hline Dasturella grewiae (Pat. \& Har.) Thirum. & Uredopeltis chevalieri J. Walker \& R.G. Shivas \\
\hline Didymopsorella toddaliae (Petch) Thirum. & Skierka toddaliae (Petch) Hirats., \\
\hline $\begin{array}{l}\text { Elateraecium divinum (Syd.) Thirum., F. Kern \& } \\
\text { B.V. Patil }\end{array}$ & Kweilingia divina (Syd.) Buriticá \\
\hline $\begin{array}{l}\text { Endophyllum tuberculatum (Ellis \& Kellerm.) Arthur } \\
\text { \& Fromme }\end{array}$ & $\begin{array}{l}\text { Pucciniosira tuberculata (Ellis \& Kellerm.) Buriticá \& } \\
\text { J.F. Hennen }\end{array}$ \\
\hline Frommea obtusa (F. Strauss) Arthur & Phragmidium potentillae (Pers.) P. Karst. \\
\hline Gymnosporangium distortum Arthur \& Cummins & Roestelia distorta (Arthur \& Cummins) F. Kern \\
\hline Hamaspora benguetensis Syd. & Hamaspora rubi-sieboldii (Kawagoe) Dietel \\
\hline Hemileia woodii Kalchbr. \& Cooke & Puccinia woodii (Kalchbr. \& Cooke) P. Syd. \& Syd. \\
\hline Jacksoniella holwayi (H.S. Jacks.) Kamat \& Sathe & Phragmidiella holwayi (H.S. Jacks.) Buriticá \\
\hline Kamatomyces narasimhanii (Thirum.) Sathe & Masseeella narasimhanii Thirum. \\
\hline $\begin{array}{l}\text { Kuehneola aliena (Syd., P. Syd. \& E.J. Butler) P. } \\
\text { Syd. \& Syd. \& E.J. Butler }\end{array}$ & $\begin{array}{l}\text { Phragmidiella aliena (Syd., P. Syd. \& E.J. Butler) } \\
\text { Buriticá \& J.F. Hennen }\end{array}$ \\
\hline $\begin{array}{l}\text { Kuehneola trichosanthis (Petch) T.S. Ramakr. \& } \\
\text { Sundaram }\end{array}$ & $\begin{array}{l}\text { Cerotelium trichosanthis (Petch) Nag Raj, Govindu \& } \\
\text { Thirum. }\end{array}$ \\
\hline Kuehneola vitis (E.J. Butler) P. Syd. \& Syd. & Chrysomyxa vitis E.J. Butler \\
\hline Melampsora laricis-epitea Kleb. & Melampsora epitea Thüm. \\
\hline $\begin{array}{l}\text { Nyssopsora schefflerae Ramachar, Bagyan., Subbal. } \\
\& \text { Hosag. }\end{array}$ & Nyssopsora thwaitesii (Berk. \& Broome) Syd. \\
\hline Ochropsora sorbi Dietel & Ochropsora ariae (Fuckel) Ramsb. \\
\hline Oplophora cedrelae (Hori) Syd. & Nyssopsora cedrelae (Hori) Tranzschel \\
\hline Phakopsora apoda (Har. \& Pat.) Mains & Angiopsora apoda (Har. \& Pat.) Aime \& McTaggart. \\
\hline Phakopsora gossypii (Lagerh.) Hirats. f. & Phakopsora desmium (Berk. \& Broome) Cummins \\
\hline Phakopsora grewiae (Pat. \& Har.) Cummins & Uredopeltis chevalieri J. Walker \& R.G. Shivas \\
\hline
\end{tabular}


Table 3 Continued.

\begin{tabular}{|c|c|}
\hline Old Name & New Name \\
\hline Phakopsora tecta H.S. Jacks. \& Holw. & Uromyces spegazzinii (De Toni) Arthur \\
\hline Phakopsora vignae (Bres.) Arthur & Phakopsora pachyrhizi Syd. \& P. Syd. \\
\hline Phakopsora ampelopsidis Dietel \& P. Syd. & $\begin{array}{l}\text { Neophysopella ampelopsidis (Dietel \& P. Syd.) Jing X. Ji } \\
\text { \& Kakish. }\end{array}$ \\
\hline Phragmidium disciflorum (Tode) J. James & Phragmidium mucronatum (Pers.) Schltdl. \\
\hline Phragmidium rubi (Pers.) G. Winter & Phragmidium bulbosum (Fr.) Schltdl. \\
\hline Puccinia absinthii DC. & Puccinia chrysanthemi Roze \\
\hline Puccinia abutili Berk. \& Broome & Puccinia abutilonis Berk. \& Broome \\
\hline Puccinia allii (DC.) F. Rudolphi & Puccinia porri (Sowerby) G. Winter \\
\hline Puccinia artemisiella P. Syd. \& Syd. & Puccinia tanaceti $\mathrm{DC}$. \\
\hline $\begin{array}{l}\text { Puccinia arthraxonis (Henn.) Syd., P. Syd. \& E.J. } \\
\text { Butler }\end{array}$ & Kuehneola loeseneriana (Henn.) H.S. Jacks. \& Holw. \\
\hline Puccinia bullata (Pers.) J. Schröt. & Puccinia angelicae (Schumach.) Fuckel \\
\hline Puccinia calcitrapae var. centaureae (DC.) Cummins & Puccinia carthami Corda \\
\hline Puccinia carduorum Jacky & Puccinia calcitrapae DC. \\
\hline Puccinia caricis Rebent. & Puccinia dioicae Magnus \\
\hline Puccinia coronata f. agrostidis Erikss. & Puccinia coronata Corda \\
\hline Puccinia coronata var. himalensis Barclay & Puccinia coronata Corda \\
\hline Puccinia dispersa Erikss. \& Henning & Puccinia recondita Roberge ex Desm. \\
\hline Puccinia epilobii-tetragoni G. Winter & Puccinia pulverulenta Grev. \\
\hline Puccinia extensicola Plowr. & Puccinia dioicae Magnus \\
\hline Puccinia glumarum (J.C. Schmidt) Erikss. \& Henning & Puccinia striiformis Westend. \\
\hline Puccinia graminis var. tritici Erikss. \& Henning & Puccinia graminis Pers. \\
\hline Puccinia himalensis (Barclay) Dietel & Chrysomyxa himalensis Barclay \\
\hline Puccinia leptodermidis (Barclay) Sacc. & Coleosporium leptodermidis (Barclay) P. Syd. \& Syd. \\
\hline $\begin{array}{l}\text { Puccinia levis var. panici-sanguinalis (Rangel) } \\
\text { Ramachar \& Cummins }\end{array}$ & Uromyces panici-sanguinalis Rangel \\
\hline Puccinia lolii E. Nielsen & Puccinia coronata Corda \\
\hline Puccinia lycoctoni Fuckel & Uromyces dactylidis G.H. Otth \\
\hline Puccinia maydis Bérenger & Puccinia sorghi Schwein. \\
\hline Puccinia obtegens (Link) Tul. & Puccinia suaveolens (Pers.) Rostr. \\
\hline $\begin{array}{l}\text { Puccinia orientalis (Syd., P. Syd. \& E.J. Butler) } \\
\text { Arthur \& Cummins }\end{array}$ & Diorchidium orientale Syd., P. Syd. \& E.J. Butler \\
\hline $\begin{array}{l}\text { Puccinia orientalis (Syd., P. Syd. \& E.J. Butler) } \\
\text { Arthur \& Cummins }\end{array}$ & Diorchidium orientale Syd., P. Syd. \& E.J. Butler \\
\hline Puccinia penniseti Zimm. & Puccinia substriata Ellis \& Barthol. \\
\hline Puccinia persistens Plowr. & Puccinia recondita Roberge ex Desm. \\
\hline Puccinia poae-nemoralis G.H. Otth & Puccinia brachypodii G.H. Otth \\
\hline Puccinia polygoni Alb. \& Schwein. & Puccinia polygoni-amphibii Pers. \\
\hline Puccinia prunicolor Syd., P. Syd. \& E.J. Butler & Puccinia purpurea Cooke \\
\hline Puccinia pruni-persicae Hori & Leucotelium pruni-persicae (Hori) Tranzschel \\
\hline Puccinia pruni-spinosae Pers. & Tranzschelia pruni-spinosae (Pers.) Dietel \\
\hline Puccinia pulsatillae Kalchbr. & Puccinia ustalis Berk. \\
\hline Puccinia punctiformis (F. Strauss) Röhl. & Puccinia suaveolens (Pers.) Rostr. \\
\hline Puccinia rubigo-vera var. tritici (Erikss.) Carleton & Puccinia striiformis Westend. \\
\hline Puccinia rubigo-vera (DC.) G. Winter & Puccinia recondita Roberge ex Desm. \\
\hline Puccinia saxifragae-micranthae Barclay & Puccinia heucherae (Schwein.) Dietel \\
\hline Puccinia substriata var. indica Ramachar \& Cummins & Puccinia substriata Ellis \& Barthol. \\
\hline $\begin{array}{l}\text { Puccinia substriata var. penicillariae (Speg.) } \\
\text { Ramachar \& Cummins }\end{array}$ & Puccinia substriata Ellis \& Barthol. \\
\hline Puccinia taraxaci Plowr. & Puccinia hieracii (Röhl.) H. Mart. \\
\hline $\begin{array}{l}\text { Puccinia tricholaenae (Syd. \& P. Syd.) T.S. Ramakr. } \\
\& \text { K. Ramakr. }\end{array}$ & Diorchidium tricholaenae Syd. \& P. Syd. \\
\hline Puccinia triticina Erikss. & Puccinia recondita Roberge ex Desn \\
\hline
\end{tabular}


Table 3 Continued.

\begin{tabular}{|c|c|}
\hline Old Name & New Name \\
\hline Puccinia anomala Rostr. & Puccinia hordei G.H. Otth \\
\hline Puccinia anthistiriae Barclay & Puccinia graminis Pers. \\
\hline Puccinia baryi (Berk. \& Broome) G. Winter & Puccinia brachypodii G.H. Otth \\
\hline Puccinia brizae-maximae T.S. Ramakr. & Puccinia graminis Pers. \\
\hline Puccinia bupleuri-falcati (DC.) G. Winter & Puccinia bupleuri F. Rudolphi \\
\hline Puccinia cichorii Bellynck ex J. Kickx f. & Puccinia hieracii (Röhl.) H. Mart. \\
\hline Ravenelia berkeleyi Mundk. \& Thirum. & Ravenelia cassiicola G.F. Atk. \\
\hline Ravenelia breyniae Syd. \& P. Syd. & Kernkampella breyniae (Syd. \& P. Syd.) Rajendren \\
\hline Ravenelia breyniae-patentis Mundk. \& Thirum. & $\begin{array}{l}\text { Kernkampella breyniae-patentis (Mundk. \& Thirum.) } \\
\text { Rajendren }\end{array}$ \\
\hline Ravenelia emblicae Syd. \& P. Syd. & Kernkampella emblicae (Syd. \& P. Syd.) G.F. Laundon \\
\hline Ravenelia evernia Syd. & Ravenelia fragrans Long \\
\hline Ravenelia kirganeliae Mundk. \& Thirum. & $\begin{array}{l}\text { Kernkampella kirganeliae (Mundk. \& Thirum.) G.F. } \\
\text { Laundon }\end{array}$ \\
\hline Ravenelia phyllanthi Mundk. \& Thirum. & $\begin{array}{l}\text { Kernkampella phyllanthi (Mundk. \& Thirum.) G.F. } \\
\text { Laundon }\end{array}$ \\
\hline Scopella aulica (Syd.) Mundk. \& Thirum. & Maravalia aulica (Syd.) Y. Ono \\
\hline Scopella echinulata (Niessl) Mains & Maravalia echinulata (Niessl ex Rabenh.) Y. Ono \\
\hline Scopella fici Mundk. \& Thirum. & Maravalia fici (Mundk. \& Thirum.) Y. Ono \\
\hline Scopella gentilis (Syd.) Mundk. \& Thirum. & Maravalia gentilis (Syd.) Y. Ono \\
\hline Scopella mimusops (Cooke) Cummins & Maravalia mimusops (Cooke) Y. Ono \\
\hline Stakmania formosana (Syd. \& P. Syd.) Sathe & Phakopsora formosana Syd. \& P. Syd. \\
\hline Teloconia rosae (Kuntze) Syd. & $\begin{array}{l}\text { Phragmidium kamtschatkae (H.W. Anderson) Arthur \& } \\
\text { Cummins }\end{array}$ \\
\hline $\begin{array}{l}\text { Thekopsora gaultheriae (Syd. \& P. Syd.) P. Syd. \& } \\
\text { Syd. }\end{array}$ & Pucciniastrum gaultheriae Syd. \& P. Syd. \\
\hline Trachyspora intrusa (Grev.) Arthur & Trachyspora alchemillae (Pers.) Fuckel \\
\hline Tranzschelia punctata Arthur & Tranzschelia pruni-spinosae (Pers.) Dietel \\
\hline Tunicopsora bagchii Suj. Singh \& P.C. Pandey & Kweilingia bagchii (Suj. Singh \& P.C. Pandey) Buriticá \\
\hline Uredo artocarpi Berk. \& Broome & Physopella artocarpi (Berk. \& Broome) Arthur \\
\hline Uredo bombacis Petch & Calidion bombacis (Petch) D.J. Soares \& R.W. Barreto \\
\hline Uredo callicarpae Petch & Uromyces callicarpae (Petch) Fujik. ex S. Ito \\
\hline Uredo colebrookeae Barclay & Olivea colebrookeae (Barclay) Thirum. \& Yadav \\
\hline Uredo fici Castagne & Cerotelium fici (Castagne) Arthur \\
\hline Uredo gaultheriae (Syd. \& P. Syd.) Hirats. f. & Pucciniastrum gaultheriae Syd. \& P. Syd. \\
\hline Uredo ipomoeae Yadav & Coleosporium ipomoeae (Schwein.) Burrill \\
\hline Uredo plumeriiae Pravenna, Nasheema \& Balakrishna & Coleosporium plumeriae Pat. \\
\hline Uredo tephrosiicola Henn. & Ravenelia tephrosiicola (Henn.) Hirats. f. \\
\hline Uredo ziziphi Pat. & Macabuna ziziphi (Pat.) Buriticá \& J.F. Hennen \\
\hline $\begin{array}{l}\text { Uredopeltis boswelliae (Patel, Payak \& N.B. Kulk.) } \\
\text { Sathe }\end{array}$ & Dasturella boswelliae Patel, Payak \& N.B. Kulk. \\
\hline $\begin{array}{l}\text { Uredopeltis boswelliae (Patel, Payak \& N.B. Kulk.) } \\
\text { Sathe }\end{array}$ & Dasturella boswelliae Patel, Payak \& N.B. Kulk. \\
\hline Uromyces fabae (Pers.) de Bary & Uromyces viciae-fabae (Pers.) J. Schröt. \\
\hline Uromyces leptodermus Syd. \& P. Syd. & Uromyces setariae-italicae Yoshino \\
\hline Uromyces linearis Berk. \& Broome & Puccinia peradeniyae Demers \& Castl. \\
\hline Uromyces lycoctoni (Kalchbr.) Trotter & Uromyces dactylidis G.H. Otth \\
\hline Uromyces phaseoli G. Winter & Uromyces appendiculatus (Pers.) Link \\
\hline Uromyces pisi (DC.) G.H. Otth & Uromyces pisi-sativi (Pers.) Liro \\
\hline Uromyces scillarum (Grev.) G. Winter & Uromyces muscari (Duby) Niessl \\
\hline Uromyces scirpi Burrill & Uromyces lineolatus (Desm.) J. Schröt. \\
\hline Uromyces sojae (Henn.) Syd. \& P. Syd. & Phakopsora pachyrhizi Syd. \& P. Syd. \\
\hline Xenostele indica Thirum. & Xenostele litseae (Pat.) Syd. \& P. Syd. \\
\hline
\end{tabular}




\section{Discussion}

This study provides an outline for rust fungi of India based on the literature. It provides complete information of Indian Pucciniales in one compilation as 69 genera and 640 species belonging to 16 families. Rust fungi are one of the extensively studied fungal groups of India, as evident from the number of researchers who have investigated these fungi since pre-independance. The outline presented in this study helps to better understand the taxonomy of Indian rust fungi. In addition to broadly studied fungal group of India, rust fungi possessed a broad host range and distribution too. As per earlier reports, rust fungi cause diseases on various plant hosts (Misra et al. 1975, Bisht \& Srivastava 1990, Cummins \& Hiratsuka 2003, Jiao et al. 2016). High relative humidity and dense forest cover might be the possible reasons to promote these rust fungi in these regions to cause diseases. The occurrence of 167 species of rust fungi belonging to 23 genera and 11 families on 170 plant species belonging to 52 families from Himachal Pradesh justified their diversity in this hilly state. Similarly, the broader host range from Poaceae with highest number of records followed by Ranunculaceae, Rosaceae, Asteraceae, Polygonaceae, Fabaceae, Salicaceae, Acanthaceae, Lamicaeae, Pinnaceae, Apiaceae, Rubiaceae, Saxifragaceae, Cyperaceae, Euphorbiaceae, Berberidiaceae, Geraniaceae, Linaceae and Zinziberaceae support a wide distribution of these fungi (Gautam \& Avasthi 2019). The occurence of 12 rust genera with 35 species belonging to 7 families on large number of herbaceous, shrubby plants including climbers, grass and trees also support the diversity and distribution of rust fungi in Himalayan regions (Singh \& Palni 2011). However, these fungi are not only limited to hilly regions of India; Mohanan (2010) documented a total of 95 rust fungi belonging to 25 genera associated with 117 forest plant species belonging to 80 host genera under 43 host families from the Western Ghats regions of Kerala. Similarly, the checklist of the rust genus Uromyces was documented by Gautam \& Avasthi (2017b), also support broad diversity of these fungi on wide-ranging host range.

Besides the diversity and distribution, rust fungi cause very devastating diseases on various agricultural crops in India. Wheat rusts caused by three species of Puccinia namely, stripe rust (by $P$. striiformis f. sp. tritici Westend.), stem rust (by P. graminis Pers. f. sp. tritici Eriks. \& Henn.) and leaf rust (caused by $P$. triticina Eriks.) pose a threat to global wheat production. The detection of Ug99 led the global community to work together to combat this disease, re-emerged as a threat and the establishment of the Borlaug Global Rust Initiative (BGRI, earlier Global Rust Initiative) in September 9, 2005. Pathotyping of rust pathogens and their identification are some important steps being deployed by researchers to find out management strategies of rust diseases. Emphasis is laid on evaluation of germplasm for rust resistance and development of various rust resistance varieties through various breeding programmes. In comparison to other crops, wheat has achieved a record high production in India during the current century. The development of rust resistance varieties has also progressed which played a vital role in protecting wheat from any epidemic threat (Tomar et al. 2014). The ICAR scientists cracked the whole genome of Puccinia triticina which is now proving very helpful in understanding the nature of this wheat rust pathogen (Kiran et al. 2016). Similarly, about 22 varieties of wheat which are are resistant to the deadly Ug99 fungal disease caused by Puccinia graminis- tritici are developed, of which, some of the varieties such as DBW 17, PBW 550, and Lok 1 are being cultivated in wheat-growing states in India (Bhardwaj et al. 2019).

Both morphological and molecular characterization of rust fungi is required to understand the natural classification and evolutionary relationship of rust fungi. Because with the difficulty of culturing of these fungi artificially, the success rate of culturing is also not so encouraging. The direct sequencing of rust fungi is also not so easy because of the isolation of DNA of other microbes associated with main rust pathogens. This may affects the quality and purity of DNA and ultimately the sequencing process and final identification. Due to all the above reasons, the information on molecular identification of Indian rust fungi is not adequate. Although a phylogeny of Indian rust fungi based on the sequence data of LSU and ITS available for corresponding rust fungi in GenBank (NCBI) is presented, the lack of molecular data for most of the rust fungi included requires the application of molecular techniques. Our phylogenetic studies based on LSU and ITS sequence data showed that few taxa of family Pucciniaceae include polyphetic taxa i.e. from Puccinia and 
Uromyces. More studies are still required for the better understanding of their taxonomic placement at different levels. Hence, fresh collections are required to generate molecular data to understand their phylogenetic relationships. This study has set the foundation for the systematics and taxonomic studies of rust fungi in India at generic and species level.

Although 69 genera of Indian rust fungi were included in this study, very few of these are known to have DNA sequence data. Molecular studies of these fungi are still scanty and there is much scope for exploratory work on this fungal group. Due to the lack of molecular studies, many genera or species need to be recollected and epitypifed, in order to place them in their correct taxonomic position. Some Indian rust fungi require much attention as there is confusion in their correct taxonomic placement. Therefore, future works are likely to focus on reassessing the samples of rust fungi reported from India on both morphological and molecular characterization. Emphasis should also be given on understanding the relationship between rust pathogens and host preference to elaborate this fungal group more precisely. In addition, a digital web based platform should be developed which help the researchers to identify Indian Pucciniales and to provide all information on their diversity, distribution and host association.

\section{Acknowledgements}

Authors are grateful to their respective organizations for providing the necessary laboratory facilities and valuable support during the study. We also thank the Anonymous reviewers for their helpful comments and suggestions.

\section{References}

Agarwal GP, Nema KG, Beliram R. 1959 - Fungi causing plant diseases at Jabalpur (M.P.) - I. Proceedings of National Academy of Sciences (India) 29, 310-315.

Agarwal GP, Sahni VP. 1964 - Fungi causing plant diseases at Jabalpur (M.P.). Mycopathologia et Mycologia Applicata 22, 245-247.

Ahmad GU. 1981. - Studies from rusts from Nagaland and Arunachal Pradesh. Indian Phyopathology 34, 240-241.

Ahmad S. 1941 - Higher fungi of Punjab plains -III. The Gasteromycetes. Journal of Indian Botanical Society 20, 135-143.

Ahmad ST. 1977 - Sehima nervosum - a new host of Puccinia versicolor. Indian Phytopathology 3, 261.

Ahmad ST, Singh S. 1969 - Addition to the wheat rust races in India-I. Race 12 \& 61 of Puccinia recondita identified during 1966 - Indian Phytopathology 22, 524-525.

Ahmad ST, Sinha VC, Mishra DP. 1969 - Addition to the wheat rust races in India. Race $14 \& 38$ of Puccinia recondita identified during 1965. Indian Phytopathology 22, 525-526.

Aime M, Bell C, Wilson A. 2018 - Deconstructing the evolutionary complexity between rust fungi (Pucciniales) and their plant hosts. Studies of Mycology 89, 143-152.

Aime MC. 2006 - Toward resolving family-level relationships in rust fungi (Uredinales). Mycoscience 47, 112-122.

Aime MC, Matheny PB, Henk DA, Frieders EM et al. 2006 - An overview of the higher-level classification of Pucciniomycotina based on combined analyses of nuclear large and small subunit rDNA sequences. Mycologia 98, 896-905.

Aime MC, McTaggart AR. 2020 - A higher-rank classification for rust fungi, with notes on genera. Fungal Systematics and Evolution 7, 21-47.

Ajrekar SL. 1912 - The caster rust (Melampsorella ricini de Toni). Journal of Bombay Natural History Society 21, 1092-1095.

Ajrekar SL. 1936 - India: New plant diseases in 1935. International Bulletin of Plant Protection 10, 122.

Ajrekar SL, Tonapy BR. 1923 - A note on life history of Uromyces aloes (Cke) Magn. Journal of Indian Botanical Society 3, 267-269. 
Alaei H, De Backer M, Nuytinck J, Maes M et al. 2009 - Phylogenetic relationships of Puccinia horiana and other rust pathogens of Chrysanthemum $\mathrm{x}$ morifolium based on rDNA ITS sequence analysis. Mycological Research 113, 668-683.

Alexopoulus CJ. 1962 - Introductory Mycology. New York: Wiley.

Ali B, Sohail Y, Mumtaz AS. 2017 - Distribution of rust fungi (Puccinia and Phragmidium) and host plants in Pakistan. Journal on New Biological Reports 6, 27-32.

Ananth KC, Chokanna NG. 1961- A contribution to the knowledge of spore dispersal of Hemileia vestratrix by certain species of thrips occurring on Coffea arabica in South India. Indian Coffee (Mon. Bulletin of Indian Coffee Bd.) 25, 37-38.

Anonymous. 1936 - Report of the tea scientific officer. United Planters Association South India pp. 131.

Anonymous. 1950 - List of common names of Indian plant diseases. Indian Journal of Agricultural Sciences 20, 107-142.

Anonymous. 1954 - Index of Fungi CMI 2(9), 165-181.

Anonymous. 1959a - Eleventh annual report of research department of Coffee Board (1957-1958).

Bulletin of Indian Coffee Board Research Department 11: 184 pp.

Anonymous. 1959b - Mycology Branch. Forest Research Institute 1952-1953. pp 67-71.

Arthur JC, Cummins GB. 1936a - Rusts of the North Western Himalayas. Mycologia 25, 397-406.

Arthur JC, Cummins GB. 1936b - Philipine rust in Clemin's collection 1923-1926-II. Philippine Journal of Science 61, 468

Arthur JC. 1917 - Relationship of the genus Kuehneola. Bulletin of Torrey Botanical Club 44, 501511.

Arthur JC. 1934 - Manual of rusts in United States and Canada, pp487, Fig XV 438, Lafayette, Indiana.

Asthana RP. 1952 - Some observations on the incidences of Uromyces cicer-arietini on Cicer arietinum. Nagpur Agruculture College Magazine 31, 20A-20B.

Bagchee KD. 1933 - Investigation on the infestation of Peridermium himalayensis Bachee on Pinus latifolia - II. Cronarium himalayensis n. sp. on Swertia sp. Indian Forest Rec. Botanical Series $18,66$.

Bagchee KD. 1950a - A contribution to our knowledge of morphology, cytology and biology of Indian coniferous rust- II, observations on the Cronatium ribicola Fischer and Peridermium indicum Colley \& Tayler on Pinus excelsa Wall in India with reference to their distributions, pathology, inoculation experiments and comparative morphology. Indian Forest Rec. Botanical Series 4, 1-41.

Bagchee KD. 1950b - A contribution to our knowledge of morphology, cytology and biology of Indian coniferous rust -III, observations on the Peridermium brevius (Barel.) Sacc. (Coleosporium barclayense sp. nov. on Senecio refinervis DC) and Melampsora oblonga sp. nov. on needles of Pinus excelsa Wall. Indian Forest Rec. Botanical Series 4, 43-64.

Bagchee KD. 1950c - Progress of forest pathology in India during quinquennium 1944-49. Indian Forester 76, 216-220.

Bagchee KD, Singh U. 1960 - Indian Forest records, New Series 1: 199-348.

Bagyanarayana G, Ravinder EJ, Ramesh P. 1998 - Prospodium tirumalensis, a new species from India. Mycotaxon 69, 473-476.

Bagyanarayana G, Ravinder EJ. 1995 - Taxonomy of Puccinia erebia on Clerodendrum (Verbenaceae). Mycotaxon 53, 433-435.

Bahadur P, Sinha S. 1967 - Possibility of new collateral hosts for the rusts of gram. Science and Culture 53, 538-539.

Bahekar VS. 1966 - Notes on some new fungi from India. Mycopathologia et Mycologia Applicata 30, 153-155.

Baiswar P, Ngachan SV, Chandra S. 2014 - Identification of Nyssopsora thwaitesii on Schefflera in northeast India. Australasian Plant Disease Notes 9, 124 
Bakshi BK, Reddy MAR, Puri YN, Singh S. 1972 - Survey of the diseases of important native and exotic forest trees in India. PL-480, report FRI Dehradun.

Bakshi BK, Singh S. 1960 - A new genus in the plant rusts. Canadian Journal of Botany 38, 259262.

Bakshi BK, Singh S. 1961 - New and noteworthy records of some mildews and rusts of Indian trees. Indian Forester 87, 542-545.

Bakshi BK, Singh S. 1972 - Suceptibility of exotic pine to Cronartium himalayense. Indian Forester 98, 239-240.

Balasubramanian KA. 1973 - Green island in the rust infection of sorghum. Current Science 42, 44441.

Barclay A. 1890a - Description of new fungus Aecidium esculentum n. sp. on Acacia eburnean Journal of Bombay Natural Society 5,161-165.

Barclay A. 1890b - Descriptive list of Uredineae occurring in neighbourhood of Simla (Western Himalaya). Journal of Asiatic Society of Bengal 1887 II. ibid 58, 232-251, 1889 ibid. 59, 75112.

Barclay A. 1890c - On some rusts and mildews of India. Journal of Botany 28, 257-261.

Barclay A. 1890d - On the history of a Himalayan Gymnosporangium (G. cunninghamianum n.sp.). Scientific Memories by Medical Officers of Army of India 5, 71-78.

Barclay A. 1890e - On the life history of Puccinia gerani-sylvatici Karst. var. himalensis. Linnaeus Botany 5, 27-36.

Barclay A. 1890f - On the life history of Uredineae on Rubia cordifolia nov.sp. Scientific Memories by Medical Officers of Army of India 5, 87-91.

Barclay A. 1891 - Additional Uredineae from the neighbourhood of Simla. Journal of Asiatic Society of Bengal 60, 211-230.

Basuchaudhary KC, Singh UP. 1971 - A sclerotial disease of Cynodon dactylon from India. Indian Phytopathology 24, 820-821.

Bauer R, Begerow D, Sampaio JP, Weiss M, Oberwinkler F. 2006 - The simple-septate basidiomycetes: a synopsis. Mycological Progress 5, 41-66.

Beenken L. 2014 - Pucciniales on Annona (Annonaceae) with special focus on the genus Phakopsora. Mycological Progress 13, 791-809.

Beenken L, Berndt R. 2010 - Rust fungi on Annonaceae: the genus Sphaerophragmium. Mycologia, 102(3), 650-663.

Beenken L, Lutz M, Scholler M. 2017 - DNA barcoding and phylogenetic analyses of the genus Coleosporium (Pucciniales) reveal that the North American goldenrod rust C. solidaginis is a neomycete on introduced and native Solidago species in Europe. Mycological Progress 16, 1073-1085.

Behera N, Mukerji KG. 1974 - Fungi of Delhi-XXV. Norway Journal of Botany 21, 1-3.

Berkeley MJ. 1839 - Description of exotic fungi in the collection of Sir W.J. Hooker from memoirs and notes of J.F. Klotzsch with additions and corrections. Annals of Natural History 3, 375401.

Berkeley MJ. 1856 - Decades of fungi, Decas 1-62 Nos. 1-620 In: Hooker's London. Journal of Botany 3-8, 1844-1856.

Berlin A, Djurle A, Samils B, Yuen J. 2012 - Genetic variation in Puccinia graminis collected from oats, rye, and barberry. Phytopathology 102, 1006-1012.

Bhagat SD, Kelkar PV. 1974 - Report of Endogone microcarpus Tul. from Maharashtra, India. Maharashtra Vigyan Mandir Patrika 9, 123-124.

Bhagyanarayana G, Ramachar P. 1985 - Spermogonial and aecial stages of Kernkampella kiragnelliae. Transactions of the British Mycological Society 84, 171-173.

Bhagyanarayana G, Rao KN. 1995 - A new species of Kuehneola on Gymnosporia montana from India. Canadian Journal of Botany 63, 762-764.

Bhardwaj SC, Singh GP, Gangwar OP, Prasad P, Kumar S. 2019 - Status of wheat rust research and progress in rust Management-Indian context. Agronomy 9, 892. 
Bhattacharya B, Baruah HK. 1953 - Fungi of Assam. Journal of University of Gauhati 4, 287-312.

Bisht GS. Srivastava SL. 1990 - Fungal diseases of some important crop plants grown in Garhwal Himalaya. In: Microbial Activity in the Himalaya, Almora (ed. Khulbe R.D.). pp. 141-153.

Blomquist CL, Scheck HJ, Haynes J, Woods PW, Bischoff J. 2014 - First published report of rust on white alder caused by Melampsoridium hiratsukanum in the United States. Plant Disease 98, 155.

Bruckart WL, Eskandari FM, Berner DK, Aime MC. 2010 - Life cycle of Puccinia acroptili on Rhaponticum (= Acroptilon) repens. Mycologia 102, 62-68.

Bubner B, Buchheit R, Friedrich F, Kummer V, Scholler M. 2019 - Species identification of European forest pathogens of the genus Milesina (Pucciniales) using urediniospore morphology and molecular barcoding including M. woodwardiana sp. nov. MycoKeys 48, 140.

Butler EJ. 1905 - Pilzkrankheitenin Indian in Jahre 1903. Zeitsch.fur Pflanzenke 15, 44-48.

Butler EJ. 1906 - Some Indian forest fungi. Indian Forester 31, 487-496, 548-556, 611-617.

Butler EJ. 1910 - A new genus of Uridinaceae. Annals of Mycology 8, 444-448.

Butler EJ. 1912 - The rust of wildvines in India. Annals of Mycology 10, 153-58.

Butler EJ. 1914 - Notes on some rusts in India. Annals of Mycology 12, 76-82.

Butler EJ. 1918- Fungi and diseases in plants. Thacker Spink \& Co, Culcutta VI, pp547.

Butler EJ, Bisby GR. 1931 - The fungi of India. Imperial Council of Agricultural Research of India.

Science Monograph I. XVIII, 1- 237pp.

Butler EJ, Bisby GR. 1960 - The Fungi of India. ICAR Publications New Delhi, India.

Butler EJ, Hayman JM. 1906 - Indian wheat rusts. Mem. Department of Agriculture of India 1: p58.

Champion HG. 1922 - Notes on the death of chir (Pinus longifolia) poles in Almora plantations of

Kashmir. Indian Forester 48, 168-174.

Chaudhari S. 1958 - Notes on fungi of Assam. Lloydia 21, 115-116.

Chavan PB. 1968 - Critical notes on rust fungi of Maharashtra, India. Sydowia 22, 292-294.

Chavan PB. 1975 - Critical notes on rust fungi of Maharashtra, India. Maharashtra Vigyan Mandir Patrika 10, 23-26.

Chavan PB, Bakare VB. 1973a - New rust from India. Maharashtra Vigyan Mandir Patrika 8, 3643.

Chavan PB, Bakare VB. 1973b - New rust from India. Maharashtra Vigyan Mandir Patrika 9, 132139.

Chavan PB, Bakare VB. 1974 - Some rust from Maharashtra. Indian Phytopathology 27, 266.

Chavan PB, Bakare VB. 1977 - Critical notes on rust fungi of western Maharashtra, India. Botanique 8, 137-140.

Chavan PB, Bhambure GB. 1975 - A rust on groundnut (Arachis hypogaea L.) from Maharashtra state, India. Maharashtra Vigyan Mandir Patrika 10, 1-2.

Chavan PB, Patil SK. 1972 - Studies in some rust fungi from India. Sydowia 26, 277-281.

Choi IY, Choi YJ, Kim JY, Shin HD. 2019 - Identification of Puccinia iridis, the rust fungus in general debt. The Korean Journal of Mycology 47, 89-94.

Chona BL, Lall G, Kakria NC. 1958 - The fungi of Delhi, ICAR Bulletin no.81, pp43.

Chona BL, Munjal RL, Kapoor JN. 1956 - Notes on the miscellaneous fungi - III. Indian Phytopathology 9, 125-132.

Chona BL, Munjal RL. 1950 - Puccinia kuehnii (Krueg.) Butler on sugarcane in India. Current Science 19, 151-152.

Chona BL, Munjal RL. 1955 - Notes on the miscellaneous fungi - II. Indian Phytopathology 8, 184198.

Chona BL, Munjal RL. 1956 - Notes on the miscellaneous fungi - III. Indian Phytopathology 9, 5356

Chowdhary S. 1948 - Some fungi from Assam - III. Indian Journal of Agriculture Sciences 18, 177 184. 
Chung WH, Tsukiboshi T, Ono Y, Kakishima M. 2004 - Phylogenetic analyses of Uromyces viciaefabae and its varieties on Vicia, Lathyrus, and Pisum in Japan. Mycoscience 45, 1-8.

Cooke MC. 1876a - Some Indian fungi. Grevillea 4, 114-118.

Cooke MC. 1876b - Some Indian fungi. Grevillea 5, 14-17.

Cooke MC. 1877 - Some parasites of Coniferae. Indian Forester 43, 88-96.

Cooke MC. 1878a - Some Himalayan fungi. Grevillea 7, 61.

Cooke MC. 1878b - Some Indian fungi. Grevillea 6, 117-118.

Cooke MC. 1879 - Some exotic fungi. Grevillea 7, 94-96.

Cooke MC. 1880a - Fungi of India. Grevillea 8, 93-96.

Cooke MC. 1880b - The genus Ravenelia. Journal of Royal Microscopic Society 3, 384-389.

Crane PE, Hiratsuka Y, Currah RS. 2000 - Reproductive biology and evidence for water dispersal of teliospores in Chrysomyxa weirii, a microcyclic spruce needle rust. Mycologia 92, 745-763.

Cummins GB. 1943 - Uredinales from North Western Himalayas. Mycologia 35, 446-458.

Cummins GB.1950 - The Scopella of the Uredinales. Bulletin of Torrey Botanical Club 77, 204213.

Cummins GB.1953 - The species of Puccinia parasitic on Andropogonaceae. Uredineana 4, 5-90.

Cunningham GH. 1931 - The rust fungi of New Zealand, together with the biology, cytology and therapeutics of the Uredinales. John McIndoe, NZ.

Cummins GB, Hiratsuka Y. 1983 - Illustrated genera of rust fungi. 1-152.

Cummins GB, Hiratsuka Y. 2003 - Illustrated Genera of Rust Fungi. 3rd edition. American Psychopathological Society, St. Paul, Minnesota, US.

Dalela GG. 1956 - Puccinia tumidepes Peck. on Lycium europaeum L. Indian Phytopathology 9, 7475.

Dalela GG, Sinha S. 1964 - Experiments on physiologic specialization on Puccinia penniseti Zimm. Indian Phytopathology 17, 61-65.

Damle VP. 1943 - A new species of Cystopus on Evolvulus alsinoides L. Journal of Indian Botanical Society 22, 133-136.

Darr GN, Shah AM. 1980 - Three new additions to Indian fungi. Indian Phytopathology 33, 608609.

Deadman ML, Al Sadi AM, Al Jahdhami S, Aime MC. 2005 - First report of rust caused by Puccinia carthami on safflower in Oman. Plant Disease 89, 208.

Deadman ML, Al Sadi AM, Al Maqbali YM, Farr DF, Aime MC. 2011 - Additions to the rust fungi (Pucciniales) from northern Oman. Sydowia 63, 155-168.

Deadman ML, Maqbali YA, Subhi AA, Yahyai RA et al. 2007 - First report of rust caused by Tranzschelia discolor on peach in Oman. Plant Disease 91, 638.

Demers JE, Liu M, Hambleton S, Castlebury LA. 2017 - Rust fungi on Panicum. Mycologia 109, 117.

Deoraj SD. 1980 - Puccinia xanthi on Xanthium strumarium in India. Indian Phytopathology 33, 483-484.

Dervis S, Dixon L, Doğanlar M, Rossman A. 2010 - Gall production on hawthorns caused by Gymnosporangium spp. in Hatay province, Turkey. Phytoparasitica 38, 391-400.

Dewan BB, Kar AK. 1974 - Fungi of Eastern Himalaya (India). Nova Hedwigia 25, 225-227.

Dietal P. 1890 - Uredineen aus dem Himalaya. Hedwigia 29, 259-270.

Dietel P. 1928 - Hemibasidii (Ustilaginales und Uredinales). An Engler, K Prantl: Die natülichen Pflanzenfamilien, Germany.

Dixon LJ, Castlebury LA, Aime MC, Glynn NC, Comstock JC. 2010 - Phylogenetic relationships of Sugarcane rust fungi. Mycological Progress 9, 459-468.

Doi 10.3390/agronomy9120892

Dube HD. 1958 - Some new hosts of rust fungi from India. Indian Phytopathology 11, 79-81.

Dube VP, Charya MU, Khurana S, Tyagi S. 1979 - New host records for two species of Uromyces. Indian Phytopathology 32, 154-155. 
Dublish PK, Singh PN. 1977 - Phytopathogenic fungi of Meerut, some new records. Current Science 46, 168.

Duplessis S, Cuomo CA, Lin YC, Aerts A et al. 2011 - Obligate biotrophy features unraveled by the genomic analysis of rust fungi. Proceedings of the National Academy of Sciences 108, 9166 1971.

Ebinghaus M, Maier W, Wingfield MJ, Begerow D. 2018 - New host associations and a novel species for the gall-inducing acacia rust genus Ravenelia in South Africa. MycoKeys 43, 1-21.

Ebinghaus M, Wingfield MJ, Begerow D, Maier W. 2020 - The genus Ravenelia (Pucciniales) in South Africa. Mycological Progress 19, 259-290.

Engkhaninun J, Ono Y, Kakishima M. 2005 - Phylogenetic relationships of four Puccinia species parasitic on Artemisia in Japan. Mycoscience 46, 61-65.

Feau N, Vialle A, Allaire M, Maier W, Hamelin RC. 2011 - DNA barcoding in the rust genus Chrysomyxa and its implications for the phylogeny of the genus. Mycologia. 103, 1250-66.

Feau N, Vialle A, Allaire M, Tanguay P et al. 2009 -Fungal pathogen (mis-) identifications: a case study with DNA barcodes on Melampsora rusts of aspen and white poplar. Mycological Research 113, 713-724.

Fernandez JL, Alvarado P. 2016 - First DNA sequences of Gymnosporangium amelanchieris and G. gracile. Boletin de la Sociedad Micologica de Madrid 40, 105-119.

Fleming J. 1874 - On some microscopic leaf fungi from the Himalayas. Microbiology Journal 12, 270-274.

Frederick RD, Snyder CL, Peterson GL, Bonde MR. 2002 - Polymerase chain reaction assays for the detection and discrimination of the Soybean rust pathogens Phakopsora pachyrhizi and $P$. meibomiae. Phytopathology 92, 217-227.

Ganguly D, Pandotra VR. 1962 - Some of the commonly occurring diseases of important medicinal and aromatic plants of Jammu and Kashmir. Indian Phytopathology 15, 50-54.

Ganguly D, Pandotra VR. 1963 - Fungi of medicinal and aromatic plants of North Western Himalayas - I. Mycopathologia et Mycologia Applicata 20, 39-40.

Gautam AK, Avasthi S. 2016a - First checklist of rust fungi in the genus Puccinia from Himachal Pradesh, India. Plant Pathology \& Quarantine 6, 106-120.

Gautam AK, Avasthi S. 2016b - Puccinia himachalensis - a new rust fungus from Himachal Pradesh, India. Plant Pathology \& Quarantine 6, 220-223.

Gautam AK, Avasthi S. 2017a - Discovery of Puccinia tiliaefolia (Pucciniales) in northwestern Himalayas, India. Polish Botanical Journal 62, 135-137

Gautam AK, Avasthi S. 2017b - Uromyces trifolii, a new addition to rust fungi of Himachal Pradesh, India with a checklist of Uromyces in India. Plant Pathology \& Quarantine 7, 1-14

Gautam AK, Avasthi S. 2017c - Fungi associated with Pistacia integerrimawith a description of a new species and one new record from India. Acta Mycologica 52, 1100.

Gautam AK, Avasthi S. 2018 - A new record to rust fungi of North Western Himalayas (Himachal Pradesh), India. Studies in Fungi 3, 234-240

Gautam AK, Avasthi S. 2019 - A checklist of rust fungi from Himachal Pradesh, India. Journal of Threatened Taxa 11, 14845-14861.

Gerdemann JW, Bakshi BK. 1976 - Endogonaceae of India, two new species. Transactions of the British Mycological Society 66, 340-342.

Gharse PS. 1944 - Life history and morphology of Trochodium ajreki Gharse. sp. nov. Journal of Indian Botanical Society 17, 141-148.

Glynn NC, Dixon LJ, Castlebury LA, Szabo LJ, Comstock JC. 2010 - PCR assays for the sugarcane rust pathogens Puccinia kuehnii and P. melanocephala and detection of a SNP associated with geographical distribution in P. kuehnii. Plant Pathology 59, 703-711.

Gokhle VP, Patel MK. 1951 - Kulkarniella - a new genus of rust. Indian Phytopathology 2, 170 173.

Gokhle VP, Patel MK. 1953 - A new species of Arthuria. Current Science 22, 246. 
Gokhle VP, Thirumalachar MJ, Patel MK. 1955 - Endophyllum sp. on Elaegnus latifolia. Current Science 22, 46.

Gopinathnair KR. 1972 - Puccinia deodikarii sp. nov. from India, Uredinales. Current Science 41, 575-576.

Goswami RN, Bhattacharjee S. 1973 - Rust, a new disease of Tejpat. Current Science 42, 257.

Goswami RN, Singh KL. 1973 - Uredinales of North East India. Indian Phytopathology 23, 310 314.

Goswami RN. 1972 - Occurrence of Aecidium vernoniae-cinereae Patch in India. Current Science $41,616$.

Goswami RN. 1974 - Rust, a new menace to groundnut in North East India. Indian Phytopathology $27,238$.

Goyal JP, Desai BG, Bhatnagar LG, Pathak VN. 1971 - Fungal collections from Rajasthan state of India. Sydowia 25, 172-175.

Gupta BK, Gupta C. 1985 - Some new fungi from Uttar Pradesh. Indian Phytopathology 38, 576.

Gupta SC. 1977 - A new disease of sugarcane in UP, India. Current Science 46, 27.

Gupta SL, Shukla TH. 1955 - Mycoflora Kanpurensis - I. Knapur Agriculture College Journal 14, $66-72$.

Hafeezkhan A. 1928a - A preliminary report on the Peridermiums of India and the occurrence of Cronartium ribicola Fisch. on Rubrum Linn. Indian Forester 54, 431-443.

Hafeezkhan A. 1928b - Inoculation of Chir (Pinus longifolia) with Colesporium companulae (Pers.) Lev. on Companula canescens Wall. and Colesporium inulae (Kunze) Ed. Fisch. on Inula cappa DC. Indian Forester 54, 176-178.

Hall TA. 1999 - BioEdit: a user-friendly biological sequence alignment editor and analysis program for Windows 95/98/NT. Nucleic Acids Symposium Series 41, 95- 98.

He MQ, Zhao RL, Hyde KD, Begerow D et al. 2019 - Notes, outline and divergence times of Basidiomycota. Fungal Diversity 1-263.

Hennings P. 1900 - Einlge Uredinean aus Verschiedenen Gebieten. Hedwigia 39, 153-155.

Henricot B, Denton G, Lane C. 2007 - First report of Puccinia heucherae on Heuchera spp. in the UK. Plant Pathology 56, 352-352.

Hiratsuka Y, Cummins GB. 1963 - Morphology of the spermogonia of the rust fungi. Mycologia 55, 487-507.

Hiratsuka Y. 1983 - The nuclear cycle and terminology of spore states in Uredinales. Mycologia 65, 432-443.

Hosagaudar VB. 1984 - A new rust of Elaeocarpus tuberculatus Roxb. from Iduki, Kerela, India. Current Science 53, 106-107.

Hosagoudar VB. 1985 - Teleomycetes of South India. Indian Phytopathology 39, 278-281.

Hrabetova M, Kolarik M, Markova J. 2015 - Phylogeny and taxonomy of grass rusts with aecia on Ranunculus and Ficaria. Mycological Progress 14, 12.

Ishaq A, Aime MC, Ayala EK, Ullah Set al. 2020 - First report of Asian pistachio rust (Pileolaria pistaciae) in Pakistan. Canadian Journal of Plant Pathology 42, 210-217.

Jadhav AN, Somani RB. 1978a - Puccinia xanthi - a report from India. Indian Phytopathology 31, 369-371.

Jadhav AN, Somani RB. 1978b - Puccinia xanthi Schev. a first report from India. Current Science 47, 905.

Jain AC, Nikam BG, Kulkarni SN, Sharma OP. 1966 - Fungi of Gwalior and Indore region. The Vikram 4, 181-187.

Jhooty JS, Sokhi SS, Bain SS, Rewal HS. 1977 - New diseases of some hosts of economic importance. India Journal of Mycology and Plant pathology 7, 162.

Jiao W, Guoying Z, Shengsong S, Yuanhao H et al. 2016 - Identification of pathogens of the Dalbergia tonkinensis rust disease. Scientia Silvae Sinicae 52, 165-169.

Jin Y, Szabo LJ, Carson M. 2010 - Century-old mystery of Puccinia striiformis life history solved with the identification of Berberis as an alternate host. Phytopathology 100, 432-435. 
Joshi LM, Lele VC. 1984 - Role of Vulpia myuros and Briza minor in the perpetuation of black rust of oats in the Nilgiri hills. Indian Phytopathology 17, 245-248.

Joshi LM, Merchand WC. 1963 - Bromus japonicas Thumb. susceptible to wheat rust under natural conditions. Indian Phytopathology 16, 312.

Joshi LM, Payak MM. 1963 - Berberis Aecidium in Lahul Valley, Western Himalayas. Mycologia $55,247-249$.

Joshi LM, Reddy AR. 1958 - Taxonomic studies on Uromyces of indigoferae species in India. Indian Phytopathology 11, 59-61.

Joshi LM, Reddy AR. 1959 - Some observations on Uromyces indigoferae Diet. \& Holw. the rust of Indigofera liniolla Retz. Indian Phytopathology 12, 25-28.

Joshi NC, Vashiist KPT. 1959 - Fungi of Ajmer (Rajasthan) - IV. Proceedings of National Academy of Sciences India 28(B), 147-150.

Joshi NC. 1958 - Fungi of Ajmer (Rajasthan) - III. Proceedings of National Academy of Sciences India 28(B), 303-307.

Kaitera J, Hiltunen R, Kauppila T, Hantula J. 2017 - Five plant families support natural sporulation of Cronartium ribicola and C. flaccidum in Finland. European Journal of Plant Patholology 149, 367-383.

Kala SP, Gaur RD. 1983a - Some new hosts and fungal record from India. Indian Phytopathology 36, 370-371.

Kala SP, Gaur RD. 1983b - Some new records of rust pathogens from Chamoli, Garhwal. Indian Phytopathology 36, 757-758.

Kamal, Singh RP. 1981 - Fungi of Gorakhpur - XXVII. Indian Journal of Mycology and Plant Pathology 11, 1-4.

Kamal, Singh S, Singh RP. 1979 - Fungi of Gorakhpur- IX. Indian Journal of Mycology and Plant Pathology 9, 245-246.

Kamil D, Sharma RK, Maheswari CU, Devi TP, Jain RK. 2013 - Checklist of Puccinia species. Division of Plant Pathology Indian Agricultural Research Institute New Delhi 110 012, India.

Kanadswamy PA, Vijyalakshmi U. 1959 - Puccinia erianthi Padwick \& Khan on cultivated sugarcane. Current Science 28, 209-210.

Kanaujia RS, Kishore R. 1981 - Annotated list of fungi from Faizabaad-V. Indian Journal of Mycology and Pathology 11, 292-293.

Kanaujia RS. 1978 - Annotated list of fungi from Faizabaad-III, Additions to the list of Indian fungi. Indian Phytopathology 30, 435-437.

Kapoor JN, Agarwal DK. 1972- Indian species of Ravenelia on Abrus and Albizia. Indian Phytopathology 25, 551-554.

Kapoor JN, Agarwal DK. 1974- Indian species of Ravenelia- I on Acacia. Indian Phytopathology 27, 666-669.

Kapooria RG, Sinha S. 1966 - Studies on host range of Uromyces fabae (Pers.) de. Bary. Indian Phytopathology 19, 229-230.

Katoh K, Standley DM. 2013 - MAFFT multiple sequence alignment software version 7: improvements in performance and usability. Molecular Biology and Evolution 30, 772-780.

Kaul B. 1959a - A new host for Melampsora heliscopiae (Pers.) Went from Kashmir. Science and Culture 24, 573-574.

Kaul TN. 1959b - Outbreaks of new records. FAO Plant Port. Bulletin 7, 54.

Kern FD, Thurston HW. 1944 - Additions to the Uredinales of Venezuala. Mycologia 36, 507.

Khanna KK, Chandra S. 1975 - A new disease of apple fruit. Plant Disease Reporter 59, 329-330.

Khanna PK. 1961 - A new report of a new host of Puccinia kanmorensis Cummins from India. Proceedings of National Academy of Sciences, India pp. 113.

Khosla HK, Puranik KK, Nema KG. 1975 - Occurrence of rust of groundnut (Puccinia arachidis Speg.) in Madhra Pradesh. JNKVV Research Journal 8(3/4), 292.

Khulbe RD, Verma BL. 1978 - A new rust record of Xanthium strumarium L. Journal of Maharashtra Agriculture University 3, 143. 
Kijpornyongpan T, Mondo SJ, Barry K, Sandor L et al. 2018 - Broad genomic sampling reveals a smut pathogenic ancestry of the fungal clade Ustilaginomycotina. Molecular Biology and Evolution 35, 1840-1854.

Kiran K, Rawal HC, Dubey H, Jaswal R et al. 2016 - Draft genome of the wheat rust pathogen (Puccinia triticina) unravels genome-wide structural variations during evolution. Genome Biology and Evolution 8, 2702-2721.

Kirk PM, Cannon PF, David JC, Stalpers JA. 2001 - Dictionary of the Fungi, 9th edn. Wallingford, UK: CABI Publishing.

Kirk PM, Cannon PF, Minter DW, Stalpers JA. 2008 - Dictionary of the Fungi (10th ed.): CAB International Wallingford, UK.

Kolmer JA, Ordonez ME, Groth JV. 2009 - The Rust Fungi. In: Encyclopedia of Life Sciences (ELS). John Wiley \& Sons, Ltd: Chichester. Doi 10.1002/9780470015902.a0021264

Kolte SJ, Awasthi RP. 1979 - Some observations on occurrence of groundnut rust in Nainital Tarai region of Uttar Pradesh. Indian Phytopatholgy 32, 155-156.

Konger G, Baruah HK. 1958 - The incidence of air borne spores in the potato plantations of upper Shilong. Journal of University of Gauhati 9, 81-89.

Krishnamurthy CS, Rangaswamy G. 1947 - Hemileia jasmine Krishnamurthy \& Rangaswamy sp. nov. on Jasminium ritchiei Clarke. Current Science 16, 31-33.

Kumar K Garg SK, Ahmad ZU. 1975 - Additions to fungi of India. Indian Journal of Mycology and Plant Pathology 5, 107.

Kwon JH, Kim J. 2014 - Asiatic dayflower rust caused by Uromyces commelinae and its phylogenetic analysis using rDNA Internal Transcribed Spacer Region. Journal of Agriculture and Life Science 48, 21-29.

Laundon GF, Ponappa KM. 1966 - A new species of Uredo on Hygrophila. Current Science 35, 492-493.

Lee SH, Lee CK, Cho SE, Shin HD. 2019 - First report of rust caused by Puccinia oxalidis on Oxalis debilis var. corymbosa in Korea. Plant Disease 103, Doi 10.1094/PDIS-05-18-0777-PDN

Lele VC. 1952 - Indian wild linseed (Linum mysorense Heyne) a possible collateral host of the rust of the cultivated linseed (Linum usitatissimum) in the hills. Indian Phytopathology 5, 144-145.

Liang YM, Tian CM, Kakishima M. 2006 - Phylogenetic relationships on 14 morphologically similar species of Pucciniastrum in Japan based on rDNA sequence data. Mycoscience 47, 137-144.

Liu M, Hambleton S. 2013 - Laying the foundation for a taxonomic review of Puccinia coronata s.l. in a phylogenetic context. Mycological Progress 12, 63-89.

Liu M, McCabe E, Chapados JT, Carey J et al. 2015 - Detection and identification of selected cereal rust pathogens by TaqMan real-time PCR. Canadian Journal Plant Pathology 37, 92-105.

Liu M, Szabo LJ, Hambleton S, Anikster Y et al. 2013 - Molecular phylogenetic relationships of the brown leaf rust fungi on Wheat, Rye, and other grasses. Plant Disease 97, 1408-1417.

Liu Y, Cao B, Tao S, Tian C, Liang Y. 2018 - Phragmidium species parasitizing species of Rosaceae in Tibet, China, with descriptions of three new species. Mycological Progress 17, 967-988.

Lutz M, Bauer R, Begerow D, Oberwinkler F. 2004 - Tuberculina - Thanatophytum/Rhizoctonia crocorum - Helicobasidium: a unique mycoparasitic-phytoparasitic life strategy. Mycological Research 108, 227-238.

Maheswari CU, Sharma RK, Kamil D, Devi TP. 2012 - Herbarium Cryptogamae Indiae Orientalis (HCIO) catalogue of fungal specimens Volume 1. Division of Plant Pathology Indian Agricultural Research Institute New Delhi 110 012, India.

Maier W, Begerow D, Weiss M, Oberwinkler F. 2003 - Phylogeny of the rust fungi: an approach using nuclear large subunit ribosomal DNA sequences. Canadian Journal of Botany 81, 12-23.

Maier W, McTaggart AR, Roux J, Wingfield MJ. 2016 - Phakopsora myrtacearum sp. nov., a newly described rust (Pucciniales) on eucalypts in eastern and southern Africa. Plant Pathology 65, $189-195$.

Maier W, Wingfield BD, Mennicken M, Wingfield MJ. 2007 - Polyphyly and two emerging lineages in the rust genera Puccinia and Uromyces. Mycological Research 111, 176-85. 
Malviya DS, Jain M. 1981 - Some new record of rust fungi. Indian Phytopathology 34, 379-380.

Manocharachary C, Rehana AR, Raghuveer Rao P. 1976 - Some interesting fungi from India. Maharashtra Vigyan Mandir Patrika 11, 38.

Manocharachary C. 1975 - New host records of some interesting fungi from India. Science and Culture 41, 237.

Marin-Felix Y, Groenewald JZ, Cai L, Chen Q et al. 2017 - Genera of phytopathogenic fungi: GOPHY 1. Studies of Mycology 86, 99-216.

Massee G. 1892 - Notes on exotic fungi in the Royal Herbarium Kew. Grevillea 21, 1-6.

Massee G. 1906 - Revision of the genus Hemileia Berk. Kew Bulletin 1906, 35-42.

Mathur PN. 1967 - On the occurrence of Uromyces sp. on Euphorbia dracunculoides in Rajasthan. Science and Culture 33, 67-68.

Mathur RS, Singh DV. 1964 - A new host of Uromyces proeminins (DC) Lev. Science and Culture 30, 605-606.

McKenzie EHC, Padamsee M, Dick M. 2013 - First report of rust on Alnus in New Zealand is Melampsoridium betulinum, not M. hiratsukanum. Plant Pathology and Quarantine 3, 59-65.

McLaughlin DJ, Frieders EM, Lu H. 1995 - A microscopist's view of heterobasidiomycete phylogeny. Studies in Mycology 38, 91-109.

Mcrae W. 1917 - Notes on South Indian Fungi. Madras Agriculture Department year book 1917, $108-111$.

McTaggart AR, Aime MC. 2018 - The species of Coleosporium (Pucciniales) on Solidago in North America. Fungal Biology 122, 800-809.

McTaggart AR, Doungsa-Ard C, Geering AD, Aime MC et al. 2015 - A co-evolutionary relationship exists between Endoraecium (Pucciniales) and its Acacia hosts in Australia. Persoonia 35, 50 62.

McTaggart AR, Shivas RG, Doungsa-ard C, Weese TL et al. 2016a - Identification of rust fungi (Pucciniales) on species of Allium in Australia. Australasian Plant Pathology 45, 581-592.

McTaggart AR, Shivas RG, van der Nest MA, Roux J et al. 2016b - Host jumps shaped the diversity of extant rust fungi (Pucciniales). New Phytologist 209, 1149-1158.

Mehta KC. 1934 - Indian Bulletin of Plant Protection 8, M202.

Mehta KC. 1940 - Further studies on cereal rust in India. Science Monograph No. 14, 224.

Mishra AK, Mishra AP. 1975 - Groundnut rust in Bihar varietal reactions. Indian Phytopathology 28, 557-559.

Mishra DP. 1963a - A new physiologic race of Indian rust in India. Indian Phytopathology 16, 102103.

Mishra DP. 1963b - Natural occurrence of the aecial stage of Puccinia sorghii Schw. on Oxalis corniculata Linn. in Nepal. Indian Phytopathology 16, 8-19.

Mishra DP, Ahmad ST, Singh S. 1965 - Natural occurrence of specialized forms of Puccinia graminis and P. striformis on Lolium perenne Indian Phytopathology 18, 214.

Mishra DP, Ahmad ST, Singh S. 1968 - A rust of Helicortichum alternating with with Thalictrum. Indian Phytopathology 21, 253-256.

Mishra DP, Lele VC. 1963 - The nature and the role of rust on Muechlenbergia hugelii Trin., a perennial grass in the Simla hills. Indian Phytopathology 16, 382-384.

Mishra DP, Prasad R. 1966 - Status of linseed rust races in India and sources of resistance. Indian Phytopathology 19, 184-188.

Mishra DP, Sharma SK, Joshi PC, Singh S. 1964 - Further studies in the crown rust of oat in India. Indian Phytopathology 17, 142-145.

Mishra DP, Sharma SK. 1963 - A new race of Puccinia reconditae Roxb. ex. Desm. in India. Indian Phytopathology 16, 313.

Mishra DP, Sharma SK. 1964 - Natural infection of Oxalis corniculata, the alternate host of Puccinia sorghi in India. Indian Phytopathology 17, 138-141.

Mishra DP, Singh S, Ahmad ST. 1975 - Notes on some rust fungi from Simla hills. Indian Phytopathology 28, 256-260. 
Mishra RP. 1969 - The Uredinae of Jabalpur (M.P.-II). Proceedings of Bihar Academy of Agriculture Sciences 17, 76-80.

Mishra RP, Khare MN. 1969 - Screening of Lathyrus germplasm collection against rust Uromyces fabae (Pers.) de Bary. Journal of Applied Science 1, 54-55.

Mishra RP, Nema KG, Singh SS. 1976 - Uredinae of Jabalpur, Madhya Pradesh-IV). JNKVV Research Journal 10, 136-139

Mishra RP, Nema KG. 1976 - Uredinae of Jabalpur (M.P.-III). JNKVV Research Journal 10, 132 135.

Misra DP, Singh S, Ahmad ST. 1975 - Notes on some rusts from Simla hills. Indian Phytopathology 28, 256-260.

Mitter JH, Tandon RN. 1930 - Fungus flora of Allahabad. Journal of Indian Botanical Society 9, 190-198.

Mitter JH, Tandon RN. 1932a - Fungus flora of Nainital - I. Journal of Indian Botanical Society 11, 178-180.

Mitter JH, Tandon RN. 1932b - Fungi of Nainital - II. Journal of Indian Botanical Society 17, 177182.

Mitter JH, Tandon RN. 1937 - Fungus flora of Allahabad, India Part-III. Proceedings of Indian Academy of Sciences 6(B), 194-201.

Mohanan C. 2010 - Rust Fungi of Kerala. Kerala, India. Kerala Forest Research Institute, Peechi, Kerela, India.

More WD, Moniz L. 1964 - Telial stage of rust on Sesbania aegyptiaca Poir. Current Science 33, 449.

Morin L, van der Merwe M, Hartley D, Muller P. 2009 - Putative natural hybrid between Puccinia lagenophorae and an unknown rust fungus on Senecio madagascariensis in KwaZulu-Natal, South Africa. Mycological Research 113, 725-736.

Mulvey RL, Hambleton S. 2015 - Stem rust of highbush-cranberry (Viburnum edule) caused by Puccinia linkii near Juneau, Alaska. Plant Disease 99, 893.

Mundkar BB. 1938 - Fungi of India - Supplement -I. ICAR Science Monograph 12, 54.

Mundkar BB. 1943 - Indian species of Phakopsora and Bubakia. Mycologia 35, 538-554.

Mundkar BB, Ahmad SS. 1946 - Revisions of and additions to Indian Fungi - II. Mycology Paper Imp. Mycology Institute 18, 11.

Mundkar BB, Kheshwala KF. 1943 - Dasturella a new genus of Uredinales. Mycologia 35, 201246.

Mundkar BB, Prasad N. 1938 - On a new Ravenelia from Indian. Mycologia 30, 635-638.

Mundkar BB, Thirumalachar MJ. 1945 - Two new genera of rust on Bignoniaceae. Mycologia 37, 619-628.

Mundkar BB, Thirumalachar MJ. 1952 - Revision and additions to Indian rust fungi - I. Mycology Paper Imp. Mycology Institute 16, 27.

Munjal RL, Kapoor JN. 1961 - Two fern rusts from India. Current Science 30, 308-310.

Munshi AH. 1976 - Polygonum demetorum L., a new host for Puccinia polygoni-abphibii Pers. Current Science 45, 314.

Mutkekar ML, Bhide VP, Patil VP. 1968 - Occurrence of a new physiologic race of Puccinia graminis-tritici (Pers.) Eriks. and P. Henn. in Maharashtra. Indian Phytopathology 21, 123 124.

Nagachan SV, Goswami RN. 1985 - Nyssopsora thirumalachari- a new rust from India. Indian Phytopathology 38, 186-187.

Nagachan SV, Verma MD.1984 - Uromyces coronatus on Zizania latifolia. Indian Phytopathology 37,741 .

Nagraj TR, Govindu HC, Thirumalachar MJ. 1971 - Some noteworthy rusts - IV. Sydowia 25, 157161.

Narasimhan MJ, Thirumalachar MJ. 1961 - Ravenelia esculenta an edible rust fungus. Phytopathology 41, 97-102. 
Narasimhan MJ, Thirumalachar MJ. 1964 - Heteroecism in Uromyces setariae-italicae, the rust on Italian millet. Mycologia 56, 554-559.

Narayan P, Kamal. 1985 - New host records from India. Indian Phytopathology 38, 394-395.

Nema KG, Agarwal GP. 1960 - Fungi causing plant disease at Jabalpur (M.P.) - IV. Proceedings of National Academy of Sciences 30(B), 55-68.

Nema KG, Mishra RP. 1965 - The Uredinales of Jabalpur M.P. Nagpur Agriculture College Magazine 6, 79.

Niskanen T, Douglas B, Kirk P, Crous P et al. 2018 - New discoveries: species of fungi described in 2017. In: K. J. Willis (ed.), State of the World's Fungi. Report. Royal Botanic Gardens, Kew. pp. 18-23.

Ono Y, Hennen J. 1983 - Taxonomy of the chaconiaceous genera (Uredinales). Transactions of the Mycological Society of Japan 24, 369-402.

Otálora MAG, Berndt R. 2018 - A taxonomic revision of the genus Puccinia on Lycieae, a tribe of Solanaceae. Mycologia 110(4): 692-709.

Padamsee M, McKenzie EHC. 2014 - A new species of rust fungus on the New Zealand endemic plant, Myosotidium, from the isolated Chatham Islands. Phytotaxa 174, 223-230.

Padamsee M, McKenzie EHC. 2017 - The intriguing and convoluted life of a heteroecious rust fungus in New Zealand. Plant Pathology 66, 1248-1257.

Padwick GW. 1945a - Notes on Indian fungi - III. Mycological Papers 12, 15.

Padwick GW. 1945b - Notes on Indian fungi - VI. Mycological Papers 17, 12.

Padwick GW, Khan A. 1944 - Notes on Indian fungi - II. Mycological Papers 10, 17.

Padwick GW, Merh JL. 1943 - Notes on Indian fungi - II. Mycological Papers 7, 7.

Pandotra VR. 1966 - Notes on fungi of Jammu and Kashmir-I. Proceedings of National Academy of Sciences 54, 68-73.

Pandotra VR, Ganguly D. 1962 - Notes on two fungi collected from Kulu Valley, Punjab. Indian Phytopathology 15, 216-217.

Pandotra VR, Ganguly D. 1964a - Fungi on medicinal and aromatic plants of North West Himalayas I. Mycopathologia et Mycologia Applicata 22, 59-68.

Pandotra VR, Ganguly D. 1964b - Fungi on medicinal and aromatic plants of North West Himalayas III. Mycopathologia et. Mycologia Applicata 22, 106-116.

Pandotra VR, Sastry KSM. 1969a - Fungi on medicinal and aromatic plants of North West Himalayas V. Proceedings of India Academy of Science 70(B), 88-89.

Pandotra VR, Sastry KSM. 1969b - Notes on the fungi of Jammu and Kashmir II. Proceedings of India Academy of Science 69(B), 207-212.

Parndekar SA. 1964 - A contribution to the fungi of Maharashtra I. Journal of University of Poona. 26, 56-64.

Patel JG, Solanki VA, Valand GB. 1985 - Rust disease of Ray ambla (Phyllantus phyllathi). Indian Phytopathology 38, 386-387.

Patel MK, Gokhle VP, Kulkarni NB. 1951a - Additions to the fungi of Bombay - I. Indian Phytopathology 4, 64-66.

Patel MK, Kamat MN, Bhide VP. 1949 - Fungi of Bombay, Supplement -I. Indian Phytopathology 2, 142-155.

Patel MK, Kamat MN, Padhya YA. 1950 - A new record of Puccinia on sugarcane in Bombay. Current Science 19, 121-122.

Patel MK, Payak MM, Kulkarni NB. 1951b - Additions to the fungi of Bombay -II. Indian Phytopathology 4, 71-73.

Patil BV, Thirumalachar MJ. 1962 - Life history and relationship of Uromyces clignyi. Indian Phytopathology 20, 225-228.

Patil BV, Thirumalachar MJ. 1964 - A new host of Puccinia versicolor. Current Science 33, 253.

Patil BV, Thirumalachar MJ. 1968 - Life history and heteroecism of Uromyces commellinae Cke. Indian Phytopathology 21, 324-330. 
Patil BV, Thirumalachar MJ. 1971 - Some new and interesting rusts from Maharashtra, India. Sydowia 25, 149-156.

Patil MS. 1977 - Studies on rusts of Maharashtra -II. Journal of Shivaji University (Science) 17, 143-147.

Patil MS, Thite AN. 1978 - Fungal flora of Amboli (Ratanagiri). Journal of Shivaji University (Science) 18, 219-224.

Patil SD. 1966a - Rust of Mahabaleshwar. The proceedings of the autumn school of botany, Mahabaleshwar, 254-261.

Patil SD. 1966b - The genus Ravenelia Berk. in Maharashtra. Maharashtra Vigyan Mandir Patrika $1,52-55$.

Patil SD, Date KG. 1977 - A new species of Ravenelia Berk. from Maharashtra. Maharashtra Vigyan Mandir Patrika 12, 21-24.

Patil SD, Date KG. 1980 - New hosts records for Puccinia and Uromyces from Maharashtra. Maharashtra Vigyan Mandir Patrika 15, 81-85.

Patwardhan PG. 1964 - A perfect stage of Uredo treminalie P. Henn. Mycopathologia et. Mycologia Applicata 24, 172-174.

Pavgi MS, Singh UP. 1969 - Morphology of pycnia of some Ravenelia species. Mycologia 61, 826830.

Pavgi MS, Upadhyay HP. 1966 - Parasitic fungi from North India - VI. Mycopathologia et. Mycologia Applicata 30, 257-260.

Pawar IS, Kulkarni UK. 1973 - Occurrence of telial stage of the rust Cerotelium fici (Cast.) Butl. in Maharashtra. Journal of Shivaji University 6, 75-77.

Payak MM. 1949 - Some parasitic fungi collected in the vicinity of Banaras. Indian Phytopathology 2, 190-193.

Payak MM. 1953 - Some records of fungi from Bombay state. Science and Culture 18, 342-343.

Payak MM. 1962 - Natural occurrence gram rust in uredial stage on Trigonella polycerata L. in Simla hills. Current Science 31, 433-434.

Payak MM. 1965 - Berberis as aecial host of Puccinia brachypodii in Simla hills, India. Phytopathology 52, 49-54.

Payak MM, Khanna A. 1970 - On two unrecorded races and a new biotype of Puccinia recondita. Indian Phytopathology 18, 26-28.

Payak MM, Mishra DP. 1963 - Physiologic specialization in Puccinia coronata Corda in India. Indian Phytopathology 16, 15-19.

Payak MM, Renfro BL. 1966 - Diseases of maize new to India. Indian Phytopathology 19, 122.

Pei MH, Bayon C, Ruiz C. 2005 - Phylogenetic relationships in some Melampsora rusts on Salicaceae assessed using rDNA sequence information Mycological Research 109, 401-409.

Petch T. 1912 - Ustilaginae and Uredineae of Ceylon. Annals of Research in Botany Gard Peradeniya 5, 223-256.

Ponappa KM. 1969 - On five fungi associated with coconut palm in India. Indian Phytopathology 22, 345-345.

Prasad N. 1948 - Studies on rust fungi of some wild grasses occurring in neighborhood of Simla. Indian Journal of Agriculture Sciences 18, 165-176.

Prasad N, Sharma LC, Singh RD. 1962 - Two new rusts from Rajasthan. Indian Phytopathology 15, $80-83$.

Prasada R. 1951 - Rust on wild grasses. Current Science 20, 242.

Prillinger H, Oberwinkler F, Umile C, Tlachac K et al. 1993 - Analysis of cell wall carbohydrates (neutral sugars) from ascomycetous and basidiomycetous yeasts with and without derivatization Journal of Applied Genetics 39, 1-34.

Puri YN. 1955 - Rusts and wood rotting fungi on some of the important conifers. Forest Bulletin Dehradun (NS Mycology) 179, 10pp.

Rahalkar SR. 1977 - A new rust on Para grass from India. Journal of Maharashtra Agriculture University 2, 172-174. 
Rajendran RB. 1966 - A new species of Caeoma from India. Bulletin of Torrey Botanical club 93, 4.

Rajendren RB. 1970 - Kernkampella: a new genus of the Uredinales. Mycologia 62, 837-843.

Ramachar P. 1956 - Curvularia pallescena Boed. on Aecidium urgineae sp. nov. Proceedings of $43^{\text {rd }}$ Indian Science Congress Association Agra Section B, 216.

Ramachar P. 1965 - Taxonomy of Puccinia penniseti. Journal of Indian Botanical Society 44, 218 233.

Ramachar P. 1966 - The species of Phakopsora and Physopella (rust fungi) on tribe Paniceae. Journal of Indian Botanical Society 45, 317-328.

Ramachar P, Bhagyanarayana G, Kumar A. 1978 - Additions to our knowledge of rust (Uredinales) from Hydrabaad (India)-III. Proceedings to Indian Academy of Sciences 87(B), 113-118.

Ramachar P, Bhagyanarayana G. 1976 - Nomenclatural changes in some Uredinales. Transactions of the British Mycological Society 67, 139-140.

Ramachar P, Bhagyanarayana G. 1977a - A new species of Melampsora from Andhra Pradesh. Current Science 46, 315-316.

Ramachar P, Bhagyanarayana G. 1977b - Mycological notes on some rust fungi from India. Mycologia 69, 1076-1079.

Ramachar P, Bhagyanarayana G. 1979 - Ramakrishnania, a new genus of Uredinales from Southern India. Canadian Journal of Botany 57, 783-786.

Ramachar P, Cummmins GB. 1965 - The species of Puccinia on Paniceae. Mycopathologia et. Mycologia Applicata 25, 7-60.

Ramakrishna V, Subbayya J. 1973 - Occurrence of groundnut rust in India. Indian Phytopathology 26, 574-575.

Ramakrishnan K, Subramanian CV. 1952 - The Fungi of India- a second supplement. Journal of Madras University 22(B), 1-65.

Ramakrishnan TS. 1950 - Some interesting rusts of South India. Indian Phytopathology 3, 43-50.

Ramakrishnan TS. 1951a - Additions to rust fungi of Madras - X. Proceeding of Indian Academy of Sciences 34 (B), 63-72.

Ramakrishnan TS. 1951b - Additions to rust fungi of Madras - XI. Proceeding of Indian Academy of Sciences 34 (B), 157-164.

Ramakrishnan TS. 1951c - Two new rusts from South India. Transactions of the British Mycological Society $34,141-145$.

Ramakrishnan TS. 1952 - Additions to rust fungi of Madras - XII. Proceeding of Indian Academy of Sciences 35 (B), 111-121.

Ramakrishnan TS. 1955a - Decline of cashew nut. Indian Phytopathology 8, 58-63.

Ramakrishnan TS. 1955b - Diseases of ornamental plants of Madras state, South India. Indian Horticulture 3, 1-9, 39-45.

Ramakrishnan TS. 1956 - Notes on some fungi from South India - V. Proceeding of Indian Academy of Sciences 44 (B), 114-121.

Ramakrishnan TS. 1957a - Notes on some fungi. Proceeding of Indian Academy of Sciences 45 (B), 176-180.

Ramakrishnan TS. 1957b - Notes on some fungi from South India - VI. Proceeding of Indian Academy of Sciences 34 (b), 149-151.

Ramakrishnan TS. 1959 - Notes on some fungi from South India - VII. Proceeding of Indian Academy of Sciences 49 (B), 124-128.

Ramakrishnan TS. 1960 - Notes on some fungi from South India - VIII. Proceeding of Indian Academy of Sciences 51(B), 164-168.

Ramakrishnan TS. 1965 - Notes on some fungi from South India - IX. Proceeding of Indian Academy of Sciences 62 (B), 32-35.

Ramakrishnan TS, Narasimhalu IL. 1941 - The occurrence of Darluca filum (Biv.) Cast. on cereal rusts in South India. Current Science 10, 290-291. 
Ramakrishnan TS, Ramakrishnan K. 1946 - Additions to rust fungi of Madras - I. Proceeding of Indian Academy of Sciences 25 (B), 28-34.

Ramakrishnan TS, Ramakrishnan K. 1947 - Additions to rust fungi of Madras - II. Proceeding of Indian Academy of Sciences 25 (B), 178-187.

Ramakrishnan TS, Ramakrishnan K. 1948a - Additions to rust fungi of Madras - I. Proceeding of Indian Academy of Sciences 25 (B), 28-34

Ramakrishnan TS, Ramakrishnan K. 1948b - Additions to rust fungi of Madras - V. Proceeding of Indian Academy of Sciences 28 (B), 50-70.

Ramakrishnan TS, Ramakrishnan K. 1949 - Additions to rust fungi of Madras - VI. Proceeding of Indian Academy of Sciences 29(B), 48-58.

Ramakrishnan TS, Ramakrishnan K. 1950a - Additions to rust fungi of Madras - VII. Proceeding of Indian Academy of Sciences 32(B), 48-58.

Ramakrishnan TS, Ramakrishnan K. 1950b - Additions to rust fungi of Madras - VIII. Proceeding of Indian Academy of Sciences 32(B), 97-111.

Ramakrishnan TS, Ramakrishnan K. 1950c - Additions to rust fungi of Madras - IX. Proceeding of Indian Academy of Sciences 32 (B), 205-214.

Ramakrishnan TS, Rangaswamy G. 1948 - Uromyces acori Ramakrishnan and Rangaswamy sp. nov. on Acorus calamus. Current Science 17, 240-241.

Ramakrishnan TS, Shrinivasan KV, Sundaram NV. 1952a - Additions to rust fungi of Madras -XIII. Proceeding of Indian Academy of Sciences 36(B), 85-95.

Ramakrishnan TS, Shrinivasan KV, Sundaram NV. 1952b - Additions to rust fungi of Madras -XIV. Proceeding of Indian Academy of Sciences 37 (B), 83-95.

Ramakrishnan TS, Shrinivasan KV. 1950 - On two new rust fungi. Current Science 19, 216.

Ramakrishnan TS, Soumini CK. 1946a - A new rust on Premna mucornata Wild. Proceeding of Indian Academy of Sciences 25 (B), 35-37.

Ramakrishnan TS, Soumini CK. 1946b - Hemileia wrightiae Racib. on Wrightia tinctoria B. \& Br. \& W. tomentosa Roem. \& Scb. Current Science 15, 256-257.

Ramakrishnan TS, Sundaram NV. 1952a - A new rust on Antidesma in India. Transactions of the British Mycological Society 35, 26-28.

Ramakrishnan TS, Sundaram NV. 1952b - Notes on some fungi from South India-I. Indian Phytopathology 5, 110-113.

Ramakrishnan TS, Sundaram NV. 1953a - Additions to rust fungi of Madras - XV. Proceeding of Indian Academy of Sciences 32 (B), 187-194.

Ramakrishnan TS, Sundaram NV. 1953b - Notes on some fungi from South India - II. Indian Phytopathology 6, 27-38.

Ramakrishnan TS, Sundaram NV. 1954a - Notes on some fungi from South India - III. Indian Phytopathology 7, 61-68.

Ramakrishnan TS, Sundaram NV. 1954b - Notes on some fungi from South India - IV. Indian Phytopathology 7, 140-151.

Ramakrishnan TS, Sundaram NV. 1955a - Additions to rust fungi of Madras - XVII. Proceeding of Indian Academy of Sciences 41 (B), 189-195.

Ramakrishnan TS, Sundaram NV. 1955b - Additions to rust fungi of Madras - XVIII. Proceeding of Indian Academy of Sciences 42 (B), 58-64.

Ramakrishnan TS, Sundaram NV. 1956a - The life history of Puccinia blepharidis P. Henn. Proceeding of Indian Academy of Sciences 44 (B), 325-328.

Ramakrishnan TS, Sundaram NV. 1956b - The life history of Puccinia romagnoliana Nair \& Sacc. Proceeding of Indian Academy of Sciences 43 (B), 95-96.

Rambaut A. 2012 - FigTree version 1.4.0. Available from: http://tree.bio.ed.ac.uk/software/figtree/ (Accessed Oct 2, 2020)

Rangaswamy G, Sheshadri VS, Lucy Channamma KA. 1968 - Fungi of South India. University of Agricultural Science Banglore, 193.

Ravindra Nath V, Narahari Reddy N. 1964 - Telia rust of castor. Current Science 33, 88. 
Rebenhorst. 1878 - Fungi europaei exsiccate. Hedwigia 17, 31-176.

Rolla SR, Addala RS. 1963 - Uromyces commelinae Cooke new host plants. Science and Culture 29, 449-451.

Roy AK. 1964 - Additions to fungal flora of Assam-I. Indian Phytopathology 18, 327-334.

Roy AK. 1968 - Additions to fungal flora of Assam-II. Indian Phytopathology 21: 182-189.

Roy B, Vogler D, Bruns T, Szaro T. 1998 - Cryptic Species in the Puccinia monoica Complex. Mycologia 90, 846-853.

Roy RY, Gupta SK. 1959 - A new species of Puccinia xanthocarpi sp. nov. Proceedings of $46^{\text {th }}$ Indian Science Congress Part - III, 339.

Roy TC. 1948 - Fungi of Bengal. Bulletin of Botanical Society of Bengal 2, 134-147.

Sachan SN, Sharma MR, Chatarji R. 1980 - Additions to rust fungi of India. Indian Journal of Mycology and Plant Pathology 10,130.

Sahni ML, Chona BL. 1965 - Studies on sugarcane rust in India. Indian Phytopathology 18, 191203.

Saini LS, Chand JN. 1984 - Uromyces dactylidis on Poa annua. Indian Phytopathology 37, 586.

Saksena HK. 1930 - Fungus flora of Allahabad, Part-I. Proceedings of Indian Science Congress 17, 284.

Saksena HK. 1956 - Two new records of Uromyces sp. from India. Science and Culture 22, 337338.

Salam MA, Ramachar P. 1955 - Additions to our knowledge to the rust fungi of Hydrabad-I. Journal Indian Botanical Society 34, 191-195.

Salam MA, Ramachar P. 1956 - Additions to our knowledge to the rust fungi of Hydrabad-II. Journal Indian Botanical Society 35, 152-157.

Sanwal BD. 1951a - On some new and noteworthy Ravenelia from India. Sydowia 5, 412-417.

Sanwal BD. 1951b-Taxonomic notes on tropical fungi - I. Sydowia 5, 384-387.

Sathe AV. 1965a - A new species of Dasturella (Uredinales) from India. Sydowia 19, 148-449.

Sathe AV. 1965b - Revision of Masseella narasimhansii Thirum. (Uredinales). Sydowia 19, 187189.

Sathe AV. 1965c - Some additions to rust fungi of Maharashtra. Journal of University of Poona 30, 3-4.

Sathe AV. 1965d - Some new or revised species of Physopella (Uredinales) from India. Sydowia 19, $138-142$.

Sathe AV. 1965e-Uredopeltis boswelliae (Patel et al.) Sathe from India. Sydowia 19, 200-201.

Sathe AV. 1966a - Some additions to fungi of India. Mycopathologia et Mycologia Applicata 29: 338-339.

Sathe AV. 1966b - Some new reports of Aecidium from India. Mycopathologia et Mycologia Applicata 29, 118-123.

Sathe AV. 1966c - Stakmania- a new genus of Uredinales form India. Sydowia 20, 252-255.

Sathe AV. 1969a - A new rust record of Pennisetum typhoides Stapf and Hub. Mycologia 61, 198199.

Sathe AV. 1969b - Peridospora - a new genus of Uredinales from India. Transactions of the British Mycological Society 53, 143-145.

Sathe AV. 1972b - Taxonomic studies of the genus Cerotelium (Uredinales). Indian Phytopathology $25,76-79$.

Sathe AV.1971 - Nomenclature of common rust fungi affecting sugarcane. Current Science 40, 4243.

Sathe AV.1972a - Identity and nomenclature of soybean rust from India. Current Science 41, 264 265.

Sathe AV, Rahalkar SR. 1976 - A new rust on Polygonum glabrum from India. Current Science 45, 382.

Saumtally AS, Viremouneix TR, Ahondokpe B, Girard JCR et al. 2011 - First Report of orange rust of Sugarcane caused by Puccinia kuehnii in Ivory Coast and Cameroon. Plant Disease 95, 357. 
Savile DBO. 1971 - Coevolution of the rust fungi and their hosts. The Quarterly Review of Biology 46, 211-218.

Scholler M, Lutz M, Aime MC. 2019 - Repeated formation of correlated species in Tranzschelia (Pucciniales). Mycological Progress 18, 295-303.

Scholler M, Lutz M, Wood AR, Hagedorn G, Mennicken M. 2011 - Taxonomy and phylogeny of Puccinia lagenophorae: a study using rDNA sequence data, morphological and host range features. Mycological Progress 10, 175-187.

Seier MK, Morin L, van der Merwe M, Evans HC, Romero A. 2009 - Are the microcyclic rust species Puccinia melampodii and Puccinia xanthii conspecific? Mycological Research 113, 12711282.

Shanmugam N, Ranganathan K, Krishnamurthy CS. 1972 - A new record of rust caused by Puccinia arachidis Speg. Madras Agriculture Journal 59, 185.

Sharma AD. 1977 - Studies on seed mycoflora of some medicinal plants. Indian Journal of Mycology and Plant Pathology 7, 171-172.

Sharma BB, Mukerji SK. 1972 - A new record of rust on groundnut (Arachis hypogea) in Calcutta, W.B. Current Science 41, 229.

Sharma ND. 1975 - Some foliicolous fungi-IV. Botanique 6, 99-102.

Sharma ND, Jain AC. 1981 - Oidium grewiae sp. nov. and Phakaopsora grewiae (Pat. \& Har.) Cumm. two new diseases on Grewia asiatica from Jabalpur. Current Science 50, 133-134.

Sharma PD, Sanger DK, Garg AP. 1979 - A brachy Puccinia on Cnicus arvensis. Indian Phytopathology 32, 150.

Sharma RK, Shankar V. 1979 - Two new host records of Uredinales. Indian Journal of Forestry 2, 12.

Sharma SK, Singh S, Goel LB. 1973 - Notes on some new records of Sonalika infecting race of yellow rust in India and source of resistance. Indian Journal of Agriculture Sciences 43, 964 965.

Sharma SK, Singh S. 1964 - A new physiologic race of Puccinia striiformis West in India. Indian Phytopathology 17, 72-73.

Sharma SL. 1957 - A new report of rust (Puccinia kuehinii (Krueg) Butler) on Erianthus munja. Proceedings of Indian Academy of Sciences. 46, 126-130.

Shin HD, Lee SH, Seo ST, Lee SK. 2019 - First report of rust caused by Coleosporium zanthoxyli on Zanthoxylum piperitum in Korea. Plant Disease 103. Doi 10.1094/PDIS-06-18-1029-PDN

Shinde PA, More WD. 1975 - Outbreak of groundnut rust at Rahuri. Research Journal of Mahatma Phule Agriculture University 6, 75.

Shukla BN, Singh BP. 1976 - Occurrence of sunflower rust (Puccinia helianthi) in Madhya Pradesh. JNKVV Research Journal 10, 45-46.

Siddiqui MR. 1957 - Two noteworthy foliicolous fungi from Saugar. Bulletin of Society of University of Sagaur 9 19-20.

Siddiqui MR. 1971 - Natural incidence of Puccinia helianthi on Helianthus cucumerifolius. Torrey and Gray in India. Science and Culture 37, 151-157.

Siddiqui MR. 1972- Studies on diseases of sunflower (Helianthus annus) in India. Indian Phytopathology 25, 160-161.

Siddiqui MR. 1973 - Helianthus cucumerifolius - a new host for sunflower rust in India. Indian Phytopathology 26, 359-361.

Singh A, Palni UT. 2011 - Diversity and distribution of rust fungi in central Himalayan region. Journal of Phytology 3, 49-59.

Singh AK, Kamal. 1985 - New fungal records from India. Indian Phytopathology 38, 388.

Singh BM, Sharma YR. 1977 - Rust on garlic. FAO, Plant Protection Bulletin 25, 41-42.

Singh H, Jalan S. 1965 - A new rust on Schizandra grandiflora. Indian Phytopathology 18, $28-32$.

Singh H. 1962 - A new aecial host of Puccinia aristidae Tracy. Current Science 31, 521-522.

Singh S. 1966 - A new species in plant rusts. Sydowia 20, 261-263. 
Sinha S, Kapooria RG. 1966 - An aspect of microbial control of Bajra Rust. Indian Phytopathology 19, 127-128.

Singh S, Pandey PC. 1972 - New Melampsoridium on Mangolia. Transactions of the British Mycological Society 58, 342-344.

Sohi HS, Durgapal JC, Guganani HC. 1967 - Narenga porphyrocoma - a new host of sugarcane rust (Puccinia helianthi). Indian Phytopathology 20, 175.

Sokhi SS, Sohi SS. 1976 - Studies on rust of cowpea cuased by Uromyces phaseoli var. vignae. Indian Phytopathology 29, 99

Solanki VA, Patel JG, Patel RB. 1985 - Rust diseases of mahova (Bassia latifolia). Indian Phytopathology $38,386$.

Somani RB. 1979 - A new species of Puccinia on Cyondon. Biovigyanam 5, 81-82.

Soumini CK. 1949 - Investigation of cereal rusts-III. Puccinia purpurea Cooke. Indian Phytopathology 2, 35-38.

Souza ESC, Aime MC, Elias SG, Pinho DB et al. 2018 - Crossopsorella, a new tropical genus of rust fungi. Phytotaxa 375, 189-202.

Srivastava RC. 1979a - Additions to fungi of India. Current Science 48, 996.

Srivastava RC. 1979b - Fungi causing plant diseases at Jounpur UP-II. Indian Phytopathology 32, 289-290.

Srivastava RC. 1979c - New host record from India. Maharashtra Vigyan Mandir Patrika 14, 79-80.

Srivastava RC. 1980 - Fungi causing plant diseases at Jounpur UP-IV. Indian Phytopathology 33, 221-224.

Srivastava SL. 1982 - Some new records of rust fungi from Kedarnath Valley. Acta Botanica Indica $10,96$.

Stamatakis A. 2014 - RAxML version 8: a tool for phylogenetic analysis and post-analysis of large phylogenies. Bioinformatics 30(9), 1312-1313.

Stamatakis A, Hoover P, Rougemont J. 2008 - A rapid bootstrap algorithm for the raxml web servers. Systematic Biology 57, 758-771.

Stuteville DL, GravesWL, Dixon LJ, Castlebury LA, Minnis AM. 2010 - Uromyces ciceris-arietini, the cause of chickpea rust: new hosts in the Trifolieae, Fabaceae. Plant Disease 94, 293-297.

Subramaniam CV, Ramakrishnan K. 1956 - List of Indian Fungi 1952-56. Journal of Madras University 26 (B), 327-421.

Subramanian CV. 1986 - The progress and status of mycology in India. Proceedings of Indian Academy of Sciences 96, 379-392.

Sunderam NV, Rao AV. 1950 - A new rust disease on Garcinia indica. Science and Culture 23, 9899.

Sunderam NV, Renfro BL, Singh JP, Rachie GKO. 1966 - Reactions of the world collection of sorghum to five important diseases. Indian Phytopathology 19, 127.

Sunderam NV. 1956 - New and complete life cycle of Puccinia rufipes Diet. Indian Phytopathology 9, 133-137.

Sunderam NV. 1961 - Notes on some fungi from South India. Indian Phytopathology 14, 202-209.

Sunderam NV. 1963 - A new host record for Puccinia citrulli Syd. \& Butler. Madras Agriculture Journal 50, 479.

Sunderam NV. 1964 - Physiologic specialization in Uromyces leptodermus Syd. Indian Journal of Agriculture Sciences 34, 215-218.

Swann EC, Frieders EM, McLaughlin DJ. 2001 - Urediniomycetes. In: D. J. McLaughlin, E. J. McLaughlin and P. Lemke, eds., The Mycota. Vol. VII. Part B, Systematics and Evolution. Springer, Berlin, Heidelberg, 37-56.

Swann EC, Taylor JW. 1995a - Phylogenetic diversity of yeast-producing Basidiomycetes. Mycol Res 99, 1205-1210.

Swann EC, Taylor JW. 1995b - Phylogenetic perspectives on Basidiomycete systematics: evidence from the 18S rRNA gene. Canadian Journal of Botany 73, 862-868. 
Sydow H. 1913 - Beitrage zur Kennitius de Pilze flora Sudlichen Ostindiens. I. Annals of Mycology 11, 326-330.

Sydow H. 1914 - Beitrage zur Kennitius de Pilze flora Sudlichen Ostindiens. I. Annals of Mycology $12,424-490$.

Sydow H. 1921 - Novae fungorum species - XVII. Annales Mycologici. 19: 304-309.

Sydow H. 1922 - Uber einigie Wenig bekannte Uredineen aus dem Kew Herber. Annals of Mycology 20, 54-60.

Sydow H. 1938 - Fungi Himalayensis. Annals of Mycology 36, 437-442.

Sydow H. 1939 - Novae fungorum species. Annals of Mycology 37, 198.

Sydow H, Butler EJ. 1901 - Fungi Indiae Orientalis Part - I. Annals of Mycology 4, 424-445.

Sydow H, Mitter JH. 1933 - Fungi Indici - I. Annals of Mycology 31, 84-97.

Sydow H, Mitter JH. 1935 - Fungi Indici - II. Annals of Mycology 33, 46-71.

Sydow H, Mitter JH, Tandon RN. 1937 - Fungi Indici - III. Annals of Mycology 35, 222-243.

Sydow H, Sydow P. 1904a - Novae fungorum species. Annales Mycologici 2, 162-174.

Sydow H, Sydow P. 1904b - Monographia Uredinearum. Volume I. leipzeg, UK.

Sydow H, Sydow P. 1917 - Novae fungporum species - I. Annals of Mycology 15, 143-148

Sydow H, Sydow P. 1924 - Monographia Uredinearum. Volume XXV, leipzeg, UK.

Sydow H, Sydow P. 1911 - Novae fungorum species - VI. Annales Mycologici 9, 142-146.

Sydow H, Sydow P. 1912a - Novae fungorum species - VII. Annales Mycologici 10, 77-85.

Sydow H, Sydow P. 1912b - Novae fungorum species - VIII. Annales Mycologici 10, 405-410.

Sydow H, Sydow P, Butler EJ. 1907 - Fungi Indiae Orientalis Pars - II. Annals of Mycology 5, 485515.

Sydow H, Sydow P, Butler EJ. 1911a - Fungi Indiae Orientalis Pars - III. Annals of Mycology 9, 372-421.

Sydow H, Sydow P, Butler EJ. 1911b - Fungi Indiae Orientalis Pars - V. Annals of Mycology 14, $177-220$.

Sydow H, Sydow P, Butler EJ. 1912 - Fungi Indiae Orientalis Pars - IV. Annals of Mycology 10, 243-280.

Sydow P, Sydow H. 1915 - Monographia Uredinearum seu Specierum Omnium ad hunc usque Diem Descriptio et Adumbratio Systematica. Fratres Borntraeger, Germany.

Talbot PHB. 1971 - Fungi with Basidia and Basidiospores Usually in Fruit bodies: Eumycota Subdivision Basidiomycotina. In: Principles of Fungal Taxonomy. Palgrave, London.

Thirumalachar MJ. 1941a - A new species of Puccinia on Ocimum adscendens. Proceedings of Indian Academy of Sciences 14(B), 466-471.

Thirumalachar MJ. 1941b - A preliminary note on Melampsora parasite on Lobelia trigona Roxb. Current Science 10, 366-367.

Thirumalachar MJ. 1942a - Morphology and parasitism of Trochodium sp. nov. Journal of Indian Botanical Society 21, 59-68.

Thirumalachar MJ. 1942b - Phragmotelium mysorensis - a new rust on Indian raspberry. Proceedings of Indian Academy of Sciences 16(B), 186-193.

Thirumalachar MJ. 1943a - A new rust disease of Cardamom. Current Science 12, 231-232.

Thirumalachar MJ. 1943b - Masseella breyniae - a new species of rusts. New Phytologist 42, 4548.

Thirumalachar MJ. 1943c - Masseella narasimhanii - a new species of rust on Flugea leucopyrus Willd. Proceedings of Indian Academy of Sciences 18(B), 36-40.

Thirumalachar MJ. 1945 - Some noteworthy rusts -I. Mycologia 37, 295-310.

Thirumalachar MJ. 1947 - Some noteworthy rusts -I. Mycologia 39, 231- 248.

Thirumalachar MJ. 1949a - Critical notes on some plant rusts. Bulletin of Torrey Botanical Club 76, 339-342.

Thirumalachar MJ. 1949b - Preliminary notes on heteroecism of Puccinia versicolor. Current Science 18, 252-253.

Thirumalachar MJ. 1950a - An undescribed genus of Uredinales. Science and Culture 16, 201-211. 
Thirumalachar MJ. 1950b - Some new and interesting fungi -II. Sydowia 4, 63-73.

Thirumalachar MJ. 1950c - Some noteworthy rusts-III. Mycologia 42, 224-332.

Thirumalachar MJ. 1951 - Critical notes on some plant rusts-II. Sydowia 5, 23-29.

Thirumalachar MJ, Bhatt VV, Dhande GW, Patel MK. 1956 - Additions to the fungi of Bombay-III. Indian Phytopathology 9, 9-14.

Thirumalachar MJ, Cummins GB. 1948 - Status of Allopuccinia, Leucotelium, Edythea, and Ypsilospora. Mycologia 40, 417-422.

Thirumalachar MJ, Cummins GB. 1949 - Taxonomic significance of sporogenous basal cells in the Uredinales. Mycologia 41, 523- 526.

Thirumalachar MJ, Gopalkrishan KS. 1947 - Morphology and spore forms and heteroecism in the giant bamboo rust, Dasturella divina. Botanical Gazette 108, 371-379.

Thirumalachar MJ, Govindu HC. 1954 - Morphological and cytological studies of a bisporidial species of Endophyllum. Botanical Gazette 115, 388-391.

Thirumalachar MJ, Kern FD, Patil BV. 1966 - Elateracecium -a new genus of the Uredinales. Mycologia 58, 391-395.

Thirumalachar MJ, Mundkar BB. 1949 - Genera of rusts-II. Indian Phytopathology 2, 193-244.

Thirumalachar MJ, Mundkar BB. 1950 - Genera of rusts, Appendix. Indian Phytopathology 3, 203204.

Thirumalachar MJ, Mundkar BB. 1951 - Revision of and addition to Indian fungi-III. Mycological Papers 40, 15.

Thirumalachar MJ, Narsimhan MJ. 1947 - Studies on the morphology and parasitism Hemileia species on Rubiaceae in Mysore. Annals of Botany in Landon NS 11, 77-89.

Thirumalachar MJ, Narsimhan MJ. 1950a - Alternation of generation and heteroecism in Puccinia versicolor. Annals of Botany 14, 341-346.

Thirumalachar MJ, Narsimhan MJ. 1950b - Cytology and life history of a bisporidial Endophyllum. New Phythology 49, 117-120.

Thirumalachar MJ, Swamy BJ, Khan BAK. 1943 - Contribution to the flora of Nandi Hills, Part I, some interesting rusts and smuts. Journal of Mysore University NS Section B 3, 195-304.

Thite AN, Patil MS. 1970 - Additions to the rusts of Maharashtra. Journal of Shiva University 15, 49-51.

Tilak ST, Rao R. 1968 - Second supplement to fungi of India (1962-1967). Marathwada University, Marathwada, 1-312.

Tomar SMS, Singh SK, Sivasamy M, Vinod 2014 - Wheat rusts in India: Resistance breeding and gene deployment - A review. Indian J. Genet., 74(2): 129-156.

Tyagi RNS, Prasad N. 1972 - The morphographic studies on genus Ravenelia occurring in Rajasthan. Indian Journal of Mycology and Plant Pathology 2, 108-135.

Tyagi RNS, Prasad N. 1978 - Some new Ravenelias from Rajasthan. Science and Culture 44: 268.

Ulbitch E. 1938 - The fungi collected by German Himalaya expedition of 1937. Notizblock Botany Gartens Berlin 14, 139-150.

Unni PN, Philip VJ. 1974 - Gall formation by Puccinia thwasitesii Berk. apud. Berk. Berl. on Gendarussa vulgaris Nees. Current Science 43, 359.

Uppal BN. Patel MK, Kamat MN. 1935 - The fungi of Bombay - VIII, 1-56.

Vaheeduddin S. 1955 - Rust on grape fruit. Current Science 24, 345.

Vasudeva RS. 1948 - Report of the Head of the Division of Mycology and Plant Pathology, Scientific Report of Agriculture Research Institute New Delhi for the year (1946-47), 145-160.

Vasudeva RS. 1949 - Report of the Head of the Division of Mycology and Plant Pathology, Scientific Report of Agriculture Research Institute New Delhi for the year (1947-48), 177-190.

Vasudeva RS. 1950a - Report of the Head of the Division of Mycology and Plant Pathology, Scientific Report of Agriculture Research Institute New Delhi for the year (1948-49), 81-90.

Vasudeva RS. 1950b - Report of the Head of the Division of Mycology and Plant Pathology, Scientific Report of Agriculture Research Institute New Delhi for the year (1949-50), 55-56. 
Vasudeva RS. 1957 - Report of the Head of the Division of Mycology and Plant Pathology, Scientific Report of Agriculture Research Institute New Delhi for the year (1954-55), 87-101.

Vasudeva RS. 1958 - Report of the Head of the Division of Mycology and Plant Pathology, Scientific Report of Agriculture Research Institute New Delhi for the year (1956-57), 86-100.

Vasudeva RS. 1962 - Fungi of India, Supplement -I, ICAR New Delhi.

Venkatakrishaiya NS. 1958 - Control of fig rust caused by Cerotelium fici (Cast.) Arth. Mysore Agriculture Journal 33, 25-26.

Virtudazo E, Nakamura H, Kakishima M. 2001 - Phylogenetic Analysis of sugarcane rusts based on sequences of ITS, 5.8 S rDNA and D1/D2 regions of LSU rDNA. Journal of General Plant Pathology 67, 28-36.

Vogler DR, Bruns TD. 1998 - Phylogenetic relationships among the pine stem rust fungi (Cronartium and Peridermium spp.). Mycologia 90, 244-257.

Vu D, Groenewald M, de Vries M, Gehrmann T et al. 2019 - Large-scale generation and analysis of filamentous fungal DNA barcodes boosts coverage for kingdom fungi and reveals thresholds for fungal species and higher taxon delimitation. Studies of Mycology 92, 135-154.

Wakhloo JL. 1962 - Rust on Solanum xanthocarpum Schrad \& Wendl. Journal of Indian Botanical Society 41, 217-219.

Wang J, Zhou G, Su S, He Y et al. 2016 - Identification of pathogens of the Dalbergia tonkinensis rust Disease. Scientia Silvae Sinicae 52, 165-169.

Wani DD, Thirumalachar MJ. 1969 - Studies on Elsinoe and Sphaeloma diseases of plants in Maharashtra, India-V. Sydowia 23, 257-260.

Waraitch KS, Khatri HL. 1976 - The occurrence of pink disease and rust of plums in India. Abstract in Indian Phytopathology 29,100.

Waraitch KS, Khatri HL. 1977 - The occurrence of pink disease and rust of plums in India. Indian Journalof Mycology and Plant Pathology 7, 203-206.

Weber RW, Webster J, Engel G. 2003 - Phylogenetic analysis of Puccinia distincta and P. lagenophorae, two closely related rust fungi causing epidemics on Asteraceae in Europe. Mycological Research 107, 15-24.

Webster J. 1980 - Introduction to Fungi, 2nd edn. Cambridge: Cambridge University Press.

Wells K. 1994 - Jelly fungi, then and now. Mycologia 86, 18-48.

Wijayawardene NN, Hyde KD, Al-Ani LKT, Tedersoo L et al. 2020 - Outline of Fungi and funguslike taxa. Mycosphere 11, 1060-1456.

Wijayawardene NN, Hyde KD, Rajeshkumar KC, Hawksworth DL et al. 2017 - Notes for genera: Ascomycota. Fungal Diversity 86, 1-594.

Wijayawardene NN, Pawłowska J, Letcher PN, Krik PM et al. 2018 - Notes for genera: basal clades of Fungi (including Aphelidiomycota, Basidiobolomycota, Blastocladiomycota, Calcarisporiellomycota, Caulochytriomycota, Chytridiomycota, Entomophthoromycota, Glomeromycota, Kickxellomycota, Monoblepharomycota, Mortierellomycota, Mucoromycota, Neocallimastigomycota, Olpidiomycota, Rozellomycota and Zoopagomycota). Fungal Diversity 92, 43-129.

Yadav AS. 1953 - Contribution to the knowledge of Uredineae of Bihar- I. Indian Phytopathology 6, 86-91.

Yadav AS. 1963a - Additions to the microfungi II. Indian Phytopathology 16, 167-170.

Yadav AS. 1963b - Contribution to the knowledge of Uredineae of Bihar- III. Indian Phytopathology $16,138-142$.

Yadav AS. 1964a - Contribution to the knowledge of Uredineae of Bihar- IV. Indian Phytopathology $16,251-254$.

Yadav AS. 1964b - Uredo thelypteridis sp. nov. Current Science 33, 88-89.

Yadav AS, Thirumalachar MJ. 1955 - Contribution to the knowledge of Uredineae of Bihar. Indian Phytopathology 8, 143-149.

Yadav AS, Yadav SC. 1965 - Nuclear condition on Aecidium of Puccinia cocao Mcalp. Journal of Indian Botanical Society 44, 163-166. 
Yadav HL, Swarup J, Saxena HK. 1975 - Occurrence of groundnut rust (Puccinia arachidis Speg.) - a new record from Uttar Pradesh, India. Indian Journal of Farm Science 3, 109.

Yadav PK, Saran K. 1985 - Aecidium hartwegiae Thuem., an addition to Indian Mycoflora. Current Science 54, 941-942.

Zhao P, Liu F, Li YM, Cai L. 2016 - Inferring phylogeny and speciation of Gymnosporangium species, and their coevolution with host plants. Scientific Reports 6, 29339.

Zhao P, Qi XH, Crous PW, Duan WJ, Cai L. 2020 - Gymnosporangium species on Malus: species delineation, diversity and host alternation. 45, 68-100.

Zuluaga C, Buriticá P, Marín M. 2011 - Phylogenetic analysis of rust fungi (Uredinales) from the Colombian Andean region using 28S ribosomal DNA sequences. Revista de Biologia Tropical 59, 517-40. 